\title{
PARALLEL REAL-TIME OLAP ON CLOUD PLATFORMS
}

\author{
by \\ Xiaoyun Zhou \\ A thesis proposal submitted to \\ the Faculty of Graduate Studies and Research \\ in partial fulfillment of \\ the requirements for the degree of \\ MASTER OF SCIENCE \\ School of Computer Science \\ at \\ CARLETON UNIVERSITY
}

Ottawa, Ontario

November, 2013

(C) Copyright by Xiaoyun Zhou, 2013 


\section{Table of Contents}

$\begin{array}{ll}\text { List of Tables } & \mathbf{v}\end{array}$

List of Figures $\quad$ vi

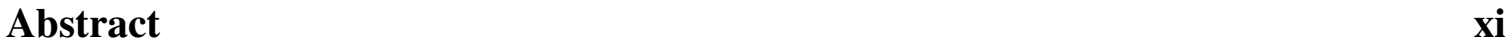

Acknowledgements $\quad$ xii

Chapter 1 Introduction 1

1.1 Introduction . . . . . . . . . . . . . . . 1

1.2 Motivation . . . . . . . . . . . . . . . . 2

1.3 Statement of the Problem . . . . . . . . . . . . . . . 3

1.4 Contributions ............................ 4

1.5 Organization of Thesis .................. 5

$\begin{array}{lll}\text { Chapter } 2 & \text { Background } & 6\end{array}$

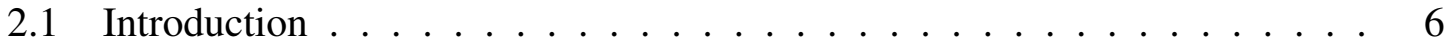

2.2 Business Intelligence and OLAP . . . . . . . . . . . 6

2.2.1 Introduction to Business Intelligence . . . . . . . . . . . 6

2.2.2 OLAP and data cube ................ 8

2.2.3 Real-time OLAP . . . . . . . . . . . . . . . 11

2.3 Parallel computing and big data processing . . . . . . . . . 11

2.3.1 Introduction to parallel computing and its advantages . . . . . . . 11

2.3.2 Use of parallel computing on large scale of data processing . . . . . 14

2.3.3 Parallel computing on cloud platforms . . . . . . . . . . . . . . . 14

2.4 Summary .............................. 15

Chapter $3 \quad$ Review of the State of the Art 16

3.1 Introduction . . . . . . . . . . . . . . . . 16 
3.2 Sequential indexes for multi-dimensional databases $\ldots \ldots \ldots \ldots$

3.3 Buiding data cubes in parallel systems . . . . . . . . . . 20

3.4 Real-time OLAP systems on cloud architectures . . . . . . . . . . 20

$\begin{array}{lll}\text { Chapter } 4 & \text { Problem Statement }\end{array}$

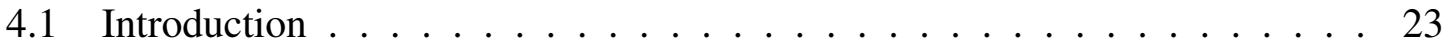

4.2 Problem statement and justification . . . . . . . . . . . . . . . . . 24

4.2.1 OLAP data size is increasing dramatically . . . . . . . . . . . 24

4.2.2 Query performance is critical . . . . . . . . . . . . . . . 24

4.2.3 Data freshness is demanded . . . . . . . . . . . . . 26

4.3 Evaluation of Results . . . . . . . . . . . . . . . . . . . . 27

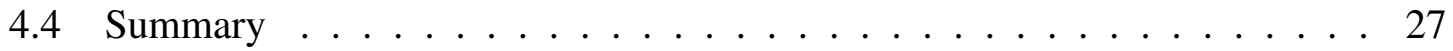

$\begin{array}{lll}\text { Chapter } 5 & \text { Parallel real-time OLAP on multi-core processors } & 28\end{array}$

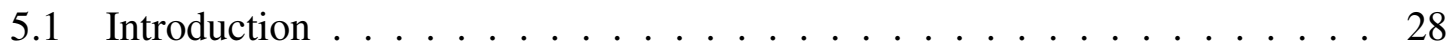

5.2 Parallel DC-Tree for multi-core processors . . . . . . . . . . . . . 28

5.3 The performance experiments of the PDC-tree on cloud platforms . . . . 31

5.3.1 Experiments environment and scope . . . . . . . . . . . 32

5.3.2 Performance evaluation . . . . . . . . . . . . . . 35

5.4 Summary $\ldots \ldots \ldots \ldots \ldots \ldots \ldots \ldots \ldots \ldots$

Chapter $6 \quad$ Real-time OLAP on cloud based distributed platform 44

6.1 Introduction . . . . . . . . . . . . . . . . . . . . . . . . . 44

6.2 Overview of PDCR-tree design . . . . . . . . . . . . . . 45

6.2.1 Distributed PDCR tree and its data structure . . . . . . . . . . 46

6.2.2 Building distributed PDCR tree from a master to workers . . . . . 52

6.2.3 The network communication Structure and message serialization . . 58

6.2 .4 Migration process and load balancer . . . . . . . . . . . . . 61

6.3 CR-OLAP operations and algorithms . . . . . . . . . . . . . 64

6.3 .1 Insertion $\ldots \ldots \ldots \ldots \ldots \ldots \ldots$

6.3 .2 Aggregation . . . . . . . . . . . . . . 66 66 
6.4 CR-OLAP query types $\ldots \ldots \ldots \ldots \ldots \ldots \ldots$

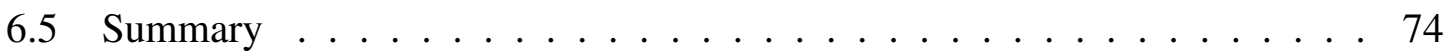

Chapter $7 \quad$ Experiment and Testing of CR-OLAP 76

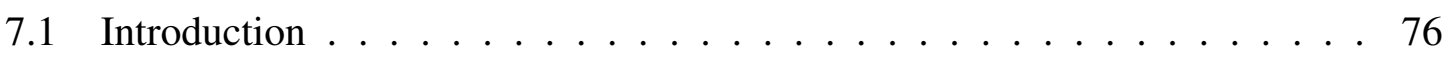

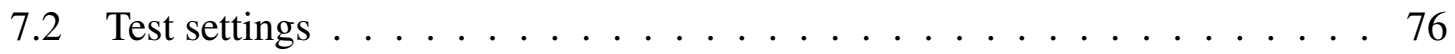

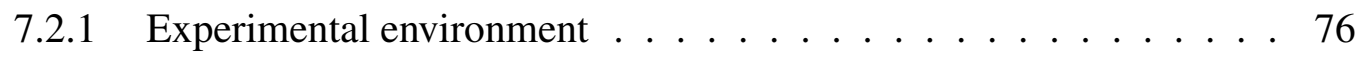

7.2 .2 Experimental data . . . . . . . . . . . . . . . . 77

7.2 .3 Comparison baseline . . . . . . . . . . . . . . 78

7.3 Analysis of Results . . . . . . . . . . . . . . . . . . . . . 79

$\begin{array}{lll}\text { Chapter } 8 & \text { Conclusions } & 91\end{array}$

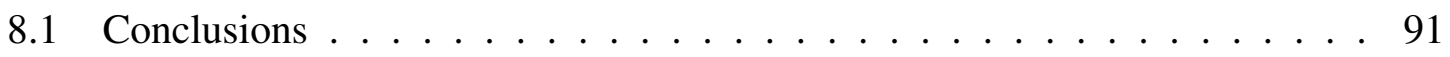

8.2 Summary of Contributions $\ldots \ldots \ldots \ldots \ldots$

8.3 Future Research . . . . . . . . . . . . . . . . . . . . . . 92

$\begin{array}{lr}\text { Bibliography } & 94\end{array}$ 


\section{List of Tables}

Table 5.1 Specifications of Amazon cloud instances used in our experiments [2] 33

Table 5.2 VCL cloud Instances . . . . . . . . . . . . . . . . 33

Table 7.1 Specifications of Amazon cloud instances used in our experiments [2] 77 


\section{List of Figures}

Figure 2.1 business intelligence architecture . . . . . . . . . . . . . 7

Figure 2.2 Snowflake schema for multi-dimensional database [14] . . . . . . . 9

Figure 2.3 a 3 -dimensional data cube $[42] \ldots \ldots$. . . . . . . . . . 9

Figure 2.4 a slice of data cube corresponding to location of Aisa [8] . . . . . 9

Figure 2.5 a dice of data cube corresponding to cellphones sold at North America in year $2000[8] \ldots \ldots$. . . . . . . . . . . . . . . 9

Figure 2.6 drill-down data cube to a specific Store 'EverMore'; (b) drill-down data cube to Store 'EverMore' and date '20080504'; (c) drill-down data cube to Store 'EverMore', month of date 'April 2008' and product category 'Food' [8] . . . . . . . . . . . . . . . . 10

Figure 2.7 Speedup by parallel computing [10] . . . . . . . . . . . . . . 12

Figure 2.8 linear, super linear and sub linear speedup [63] . . . . . . . . . 13

Figure 3.1 an R-tree for 2D rectangles [12] . . . . . . . . . . . . . . . 17

Figure 3.2 Visualization of an R-tree for 3D cubes [12] . . . . . . . . . . . 17

Figure 3.3 Structure of the X-tree . . . . . . . . . . . . . 18

Figure 3.4 Hierarchy Schema and Concept Hierarchy for dimension Customer . 18

Figure 5.1 data structure of a directory node in PDC-tree . . . . . . . . . . . 29

Figure 5.2 the structure of PDC-tree . . . . . . . . . . . . . . . . 29

Figure 5.3 (a) Node split during insertion (b) stacks used for traversing directory node for query $[26] \ldots \ldots$. . . . . . . . . . . . 30

Figure 5.4 Hierarchy shema and Conceptural Hierarchy for each dimension . . 34

Figure 5.5 Increasing the number of threads on different types of processors . . 36

Figure 5.6 Increasing the number of threads on a 2-core processor . . . . . . 36

Figure 5.7 Increasing the number of threads on a 4-cores processor . . . . . . 36

Figure 5.8 Increasing the number of threads on a 8-core processor . . . . . . . 37

Figure 5.9 Increasing the number of threads on a 16-core processor . . . . . . . 37

Figure 5.10 Increasing the number of insertions from 0 to $1000 \ldots 38$ 
Figure 5.11 increasing the size of initial tree . . . . . . . . . . . 38

Figure 5.121 thread/0 insert/1000 queries . . . . . . . . . . . . . . . 39

Figure 5.132 threads/0 insert/1000 queries . . . . . . . . . . . . . 39

Figure 5.144 threads $/ 0$ insert/1000 queries . . . . . . . . . . . . . . 39

Figure 5.158 threads $/ 200$ insert/1000 queries . . . . . . . . . . . . . . 39

Figure $5.16100 \mathrm{k}$ data Insertion and 1000 query time with $25 \%$ coverage $(5<=$ capacity $<=960) \ldots \ldots \ldots \ldots$. . . . . . . . . . . . . .

Figure $5.1750 \mathrm{k}$ data insertion and 1000 query time with $25 \%$ coverage $(5<=$ capacity $<=960) \ldots \ldots \ldots \ldots$. . . . . . . . . . 40

Figure 5.18 100k data Insertion and 1000 Query with $25 \%$ coverage $(5<=$ Capacity $<=2500) \ldots \ldots \ldots \ldots \ldots$. . . . . . . . . . .

Figure 5.19 Number of node splits, directory nodes, MDS expansion, . . . . . . 42

Figure 5.201 million data insertion and 1000 queries with $25 \%$ coverage $(10<=$ capacity $<=360) \%$ coverage $(5<=$ capacity $<=960) \quad \ldots . . .42$

Figure 5.211 million data insertion and 1000 queries with $5 \%$ coverage $(10<=$ capacity $<=480) \ldots \ldots \ldots \ldots \ldots$. . . . . . . . . . 42

Figure 6.1 System architecture . . . . . . . . . . . . . . 46

Figure 6.2 Distributed PDCR tree in master and workers . . . . . . . . . . 47

Figure 6.3 Data structure of directory node . . . . . . . . . . . . . . . . 48

Figure 6.4 Data structure of data node . . . . . . . . . . . . . . . 48

Figure 6.5 Array structure of data nodes and directory nodes . . . . . . . . 48

Figure 6.6 MDS IDs in bit representation . . . . . . . . . . . 50

Figure 6.7 An example of relationship between hierarchy levels of a given dimension ....................... 51

Figure 6.8 Vertical split during a new data insertion . . . . . . . . . 53 
Figure 6.9 PDCR-tree growing from master to a worker: (a) Tree with four data nodes; (b) Vertical node split performed when node A exceeds capacity; (c) the subtree is moved to worker when the depth of tree exceeds cut level; (d) a vertical split performed on node A in worker; (e) subtree 1 and subtree 2 becomes two independent subtrees after the vertical split and subtree $\mathrm{A}^{\prime}$ is moved to worker $2 \ldots \ldots 4$

Figure 6.10 a subtree becomes independent after vertical node split: (a) Subtree B root with sibling links; (b) Node B has vertical split and creates Node D; (c) Subtree D resulted from vertical split does not have links with neighbor nodes . . . . . . . . . . . . . 55

Figure 6.11 Subtree becomes independent to any neighbor nodes . . . . . . . . . 57

Figure 6.12 Global architecture of network communicator . . . . . . . . . . 59

Figure 6.13 Subtree migration process . . . . . . . . . . . . 63

Figure 6.14 Examples of query patterns . . . . . . . . . . . . . . . 74

Figure 7.1 Store_Sales Fact table and 8 dimensions and each with its hierarchy

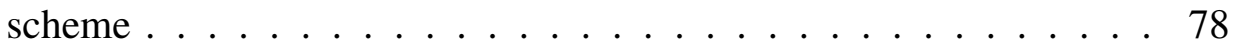

Figure 7.2 Time for 1000 insertions as a function of the number of dimensions. $(N=40 M, m=8, k=16) \ldots \ldots \ldots \ldots$

Figure 7.3 Time for 1000 queries with different query coverage, as a function of the number of dimensions. $(N=40 M, m=8, k=16) \ldots \ldots$

Figure 7.4 Time for 1000 insertions as a function of the number of workers. $(N=$ $10 M, k=16, d=8) \ldots \ldots \ldots \ldots 1$

Figure 7.5 Time for 1000 queries with different query coverages as a function of the number of workers. $(N=10 M, k=16, d=8) \ldots . . .81$

Figure 7.6 Speedup for 1000 queries with different query coverage as a function of the number of workers. $(N=10 M, k=16, d=8) \ldots \ldots 1$

Figure 7.7 Time for 1000 insertions as a function of the data size (number of items currently stored). $(k=16, d=8) \ldots \ldots$. . . . . . 82 
Figure 7.8 Time for 1000 queries with different query coverage as a function of the data size (number of items currently stored). $(k=16, d=8) \quad . \quad 82$

Figure 7.9 Time for 1000 queries as a function of query coverages in PDCRtree Impact of queries having value "**" for different dimensions. $(N=$ $40 M, m=8, k=16, d=8,10 \%<=$ Coverage $<=90 \%) \ldots . .83$

Figure 7.10 Time for 1000 queries as the query coverage in PDCR-tree Impact of queries having value "**" for different dimensions. $(N=40 M, m=$ $8, k=16, d=8,91 \%<=$ Coverage $<=99 \%) \ldots \ldots \ldots$

Figure 7.11 Time for 1000 queries as a function of query coverages in 1D-Index Impact of queries having value "*” for different dimensions. $(N=$ $40 M, m=8, k=16, d=8,10 \%<=$ Coverage $<=90 \%) \ldots 84$

Figure 7.12 Time for 1000 queries as the query coverage in 1D-Index Impact of queries having value "*”" for different dimensions. $(N=40 M, m=$ $8, k=16, d=8,91 \%<=$ Coverage $<=99 \%) \ldots \ldots . \ldots 84$

Figure 7.13 Ratio of 1D-index/PDCR-tree taken for 1000 queries as the query coverage increases . . . . . . . . . . . . . . . . 84

Figure 7.14 Ratio of 1D-index/PDCR-tree taken for 1000 queries as the query coverage increases . . . . . . . . . . . . . . . 84

Figure 7.15 Time for 1000 queries as a function of query coverage for queries with multiple “*” values for PDC-tree. $(N=40 M, m=8, k=16, d=8) 85$

Figure 7.16 Time for 1000 queries as a function of query coverages for queries with multiple “*” values for both PDCR-tree and 1D-index. $(N=$ $40 M, m=8, k=16, d=8) \ldots \ldots \ldots \ldots$

Figure 7.17 Time for 1000 queries on different type cloud instances. $(N=10 M, m=$ $4, k=16) \ldots \ldots \ldots \ldots \ldots \ldots \ldots \ldots$

Figure 7.18 Time for 1000 insertions on different type cloud instances. $(N=$ $10 M, m=4, k=16) \ldots \ldots \ldots \ldots \ldots \ldots$

Figure 7.19 Time for 1000 queries as a function of the number of cut level in hat. $(N=10 M, m=4, k=16) \ldots \ldots \ldots \ldots \ldots$ 
Figure 7.20 Time for 1000 insertions as a function of the number of cut level in hat. $(N=10 M, m=4, k=16) \ldots \ldots \ldots \ldots$

Figure 7.21 Time for 1000 queries as a function of the number of directory node capacity in hat. $(N=10 M, m=4, k=16) \ldots \ldots \ldots 8$

Figure 7.22 Time for 1000 insertions as a function of the number of directory node capacity in hat. $(N=10 M, m=4, k=16) \ldots \ldots$. . . 88

Figure 7.23 Time for 1000 queries as a function of the number of directory node capacity in workers. $(N=10 M, m=4, k=16) \ldots \ldots \ldots$

Figure 7.24 Time for 1000 insertions as a function of the number of directory node capacity in workers. $(N=10 M, m=4, k=16) \ldots \ldots$. . 89

Figure 7.25 Time for 1000 queries as a function of the number of directory node capacity in both hat and workers. $(N=10 M, m=4, k=16) \ldots \ldots 90$

Figure 7.26 Time for 1000 insertions as a function of the number of directory node capacity in both hat and workers. $(N=10 M, m=4, k=16) \ldots 90$ 


\section{Abstract}

Successful organizations increasingly rely on data analysis to develop new opportunities, guide business strategies and optimize resources. Online analytical processing (OLAP) systems are one of the most powerful technologies to provide the ability to interactively analyze multidimensional data from multiple perspectives. Traditionally, an OLAP system periodically (e.g. weekly) loads its data in batches and then builds static data cubes to answer queries quickly. However, many businesses expect OLAP systems which run in real-time, updating themselves dynamically as new data arrives. For this reason real-time OLAP systems' performance must be highly optimized.

F. Dehne and H. Zaboli in their work "A Parallel Real-Time OLAP On Multi-Core Processors" introduced the PDC-tree which works with dimension hierarchies and parallelizes insertions and queries on OLAP systems. In this thesis we designed a new data structure, the PDCR-tree, that extends the PDC-tree to work on distributed systems providing low-latency transactions processing even for very complex queries.

Using a PDCR-tree we demonstrate how to build a real-time OLAP system on a cloud based distributed platform called CR-OLAP. The CR-OLAP can be built using an $\mathrm{m}+1$ machine scalable architecture so as the system load increases, the number of machines, $\mathrm{m}$, can be increased to improve performance. Experiments show the system can process a query with $60 \%$ data coverage with on a database with 80 million data tuples with a response time 0.3 seconds or less, well within the parameters of a real-time system.

This project is funded and supported by IBM Research Canada. A research paper about this topic has been accepted by the 2013 IEEE International Conference on Big Data (IEEE BigData 2013). 


\section{Acknowledgements}

I would like to thank my supervisor, Professor Frank Dehne, and as well as project member Professor Andrew Rau-Chaplin from Dalhousie University. Both these people gave their full support to this research project. I also appreciate the collaboration of Ph.D candidate Hamed Zaboli from Carleton University, and Master student Quan Kong from Dalhousie University. Their contribution to this project was significant. Mr. Zaboli in particular gave his help generously enabling me to quickly understand the theory and practical aspects to this project. I really enjoyed working with all these people. 


\section{Chapter 1}

\section{Introduction}

\subsection{Introduction}

In a fast changing global economy to keep business competitive organizations must react to new opportunities and changes quickly. Decision makers should make their decisions fast based on the right information. Such decision making usually is assisted by computer based Decision Support Systems (DSS), which are also known as Business Intelligence (BI) applications [55]. BI is a set of methodologies or technologies that transform company's raw data into meaningful information to aid in decision-making quickly. Many organizations collect a large amount of data through day-to-day operations from different information systems, such as Enterprise Resource Planning (ERP) systems, Customer Relationship Management (CRM) systems, accounting software and sales tools. Business intelligence applications can turn source data into useable information for organizations to understand business behaviors and make strategic and tactical decisions. For example, Wendy's restaurants use BI to decide what new products should be added to their menus, and which under-performing stores should be closed. They also use BI to renegotiate contracts with food suppliers and identify opportunities to improve inefficient processes [51]. In the past few years, there is an increasing trend to use BI in many companies to identify important trends, analyze customer behaviours, and improve business processes.

One of the key components of BI system is Online Analytical Processing (OLAP). It is a tool that analyzes multi-dimensional business data providing insights supporting business decision making. In other words, OLAP enables users to view their data from different points of view. For example, a user can request data to be analyzed to show the total sales of TVs sold in city A in summer, and compare the sales of TVs sold in city B for the same time frame. The user can also compare the sales of TVs sold in city A in summer with the sales of the same product sold in city A in winter season. Databases configured for OLAP usually use a multidimensional data model. Data attributes such as product, sales region, 
and time period are treated as separate dimensions, allowing the user to swiftly perform complex analytical reports and ad-hoc queries [49]. OLAP provides the ability to model business problems enabling organizations gain better insight into the data [7].

In this thesis we discuss the limitations of current OLAP systems and propose a parallel OLAP system, which handles queries and insertions concurrently. This architecture allows users to work with real-time data and to do real-time analysis.

\subsection{Motivation}

OLAP is designed to provide consistently fast query performance to support decision making. Traditional OLAP is built on summarized tables and materialized views. The data is stored in a relational data warehouse of historical, summarized and consolidated information from possibly several operational systems. Over long periods of time, the database size can become quite large. For businesses like Walmart the data warehouse could contain terabytes or petabytes of data [4]. Summarizing the data to load into the OLAP system requires pre-computations. As a result, it may take minutes or even hours to complete an update or an insertion since it involves aggregating data along data dimensions. In current practice the data is usually updated in batches or on a periodical basis (e.g. weekly). Consequently the system is not real-time and the data used for decision support could be outof-date leading to ineffective decision making [15, 57]. For this reason real-time analysis is required by more and more businesses such as stock exchange analysis, online shopping or financial assessment.

OLAP query performance issues on a large scale data warehouses has drawn the attention of many researchers. Recently hardware has become faster and cheaper and software can perform better by running on new hardware. Applying parallelism is another way to improve software process speed. Parallel computing is popular because it improves performance by splitting large problems into smaller ones and solving them concurrently [61]. The innovation in computers with multi-cores and multi-processors, clusters with multiple computers, and cloud technologies provide the optimum platforms to support parallelism. Parallel methods and high performance parallel architecture can be applied to develop new real-time systems to support fast decision making. 
In this thesis, our goal is to design a parallel OLAP model to handle queries and insertions simultaneously on a distributed system framework (e.g. a cluster in cloud). Such a system not only runs fast on small and medium data sets but also has the scalability to handle larger data sets.

\subsection{Statement of the Problem}

The function of BI and OLAP is to assist complex data analysis in response to business queries. Usually OLAP data is stored into multidimensional data arrays so users can query the data along many dimensions [15]. As business complexity increases, the demands on OLAP also increase.

High performance, in other words, quick response, is expected by users. The process of data loading to OLAP can add much extra information on the source data in order to provide more detailed views from different angles. As a result, the data sets contained in OLAP can be very large in size. Queries against such large volume multidimensional databases will be slow without proper indexing.

Meanwhile, businesses have high demand on real-time analysis and reporting. Users should be able to perform analysis with integrated data source in real time so that the result is not based on outdated information. Usually OLAP systems update their data on a periodical basis (e.g. daily, weekly, even monthly), so the data is often out of date.

Further, system scalability or elasticity is required to support growing data volume. OLAP multi-dimensional data arrays can contain more than 3 dimensions. There is no limit to the number of dimensions that every array can contain. The pre-computation for different levels of aggregation also contributes to a data explosion. It can be very time consuming to load and maintain these huge data models. Without scalability, users will have to invest in expensive hardware to process and accommodate exploding data.

To meet those requirements, many algorithms, models, or frameworks have been discussed or proposed by researchers. We will review some of these approaches, and also provide our own approach to solve the above problems. 


\subsection{Contributions}

In this thesis both OLAP databases on multi-core processors using PDC-tree indexes and the extended version using PDCR-tree indexes for distributed cloud environments will be discussed.

The PDC-tree is able to index multi-dimensional databases with hierarchical structures and allows operations (insertions and queries) concurrently on multi-core processors. The system has been developed by our research group members Prof. Frank Dehne and Ph.D. student Hamed Zoboli. In addition to the experiments on 16 and 20 cores PCs presented in the paper "A Parallel Real-Time OLAP On Multi-Core Processors" I also conducted experiments to further evaluate PDC-tree performance on a cloud environment and the impact of system parameters.

The PDCR-tree is an index designed for a distributed system. It not only carries many properties of the PDC-tree to handle parallelism on individual processor, but also distributes multi-dimensional data to multiple processors on the cloud environment so that tasks execution can be parallelized among multiple machines. We call the system CR-OLAP. It efficiently supports OLAP query processing and data insertion concurrently and ensures that the results for an OLAP query includes all data inserted prior to the query request. Multiple experiments are provided to demonstrate the performance and show CR-OLAP provides the potential to build a real-time OLAP with a distributed system on cloud based architectures.

The project is supported by IBM Centre for Advanced Studies Canada and is conducted with the participation of Prof. Frank Dehne and Ph.D. candidate Hamed Zaboli from Carleton and Prof. Andrew Rue-Chaplin and Master student Quang Kong from Dalhousie University and myself. We conducted an extensive literature review. Mr. Zaboli provided the main idea behind the solution. We all participated in technical discussion and gave thoughts on details in the design. During the implementation of CR-OLAP, Mr. Zaboli was responsible for the PDCR-tree, Mr. Kong was responsible for the network communicator, and I was responsible for message serialization/de-serialization API, and migration API. In addition I developed code related to MDS Id used in PDCR-tree, such as ID assignment, ID upgrade and ID compare etc. Mr. Kong also developed the Stream-OLAP system which is a one dimensional index (1D-Index) structure for multidimensional database operations. 
The Stream-OLAP system provides for comparing base to CR-OLAP experiments. Mr. Zaboli conducted the first 5 experiments of the CR-OLAP provided in the Chapter 7 and I conducted the experiments on evaluating the changing system parameters of the CR-OLAP and Mr. Kong conducted the experiments for the Stream-OLAP system. I also conducted multiple tests provided in Chapter 5 to evaluate the PDC-tree system's performance in Cloud environments.

\subsection{Organization of Thesis}

The remainder of this thesis is organized as follows. In Chapter 2, we review background related to OLAP systems and parallel computing on distributed systems. Chapter 3 presents the state of art of OLAP systems and also presents some related work has been done by other researches to solve similar problems. In Chapter 4 we discuss the shortcomings of current OLAP systems that has not been solved by other researches. In Chapter 5, we present the design of parallel real-time OLAP on multi-core processors and the various experiments using different cloud environments. Chapter 6 explains our new methodology of parallel real-time OLAP designed for distributed cloud platforms and performance experiments are provided in Chapter 7. The final Chapter 8 contains concluding remarks and future work. 


\section{Chapter 2}

\section{Background}

\subsection{Introduction}

Data is always important to any industry. With the help of business intelligence applications, it becomes easier to manage, store, maintain and classify data. Therefore, organization can better use the information underlying raw data to make business decisions [61]. Many businesses benefit from BI systems. In the following, we will review the concept of BI and its key component OLAP. Since OLAP usually handles large volume of data, we will also give an introduction to parallel computing and its application to processing large data sets.

\subsection{Business Intelligence and OLAP}

\subsubsection{Introduction to Business Intelligence}

The term Business Intelligence was first defined in 1958 by IBM researcher Hans Peter Luhn[48] however only starting in the mid 1990s did the BI concept start to gain popularity. BI covers software-enabled innovations in performance management, planning, reporting, querying, online analytical processing, integration with operational systems, predictive analytics and related areas $[54,48]$. As it is understood today, BI is computer-based techniques used to analyze business data such as sales revenue by products categories or store locations or client types.

The Figure 2.1 demonstrates the components of a BI architecture. It includes ETL (extract, transform and load) engine, data warehouse, OLAP or reporting. BI applications can benefit users to understand the strengths and weaknesses of their organizations, to understand the relationship between different data for better decisions, and to discover opportunities for improvement.

Organizations use Online Transaction Processing (OLTP) based databases to implement 


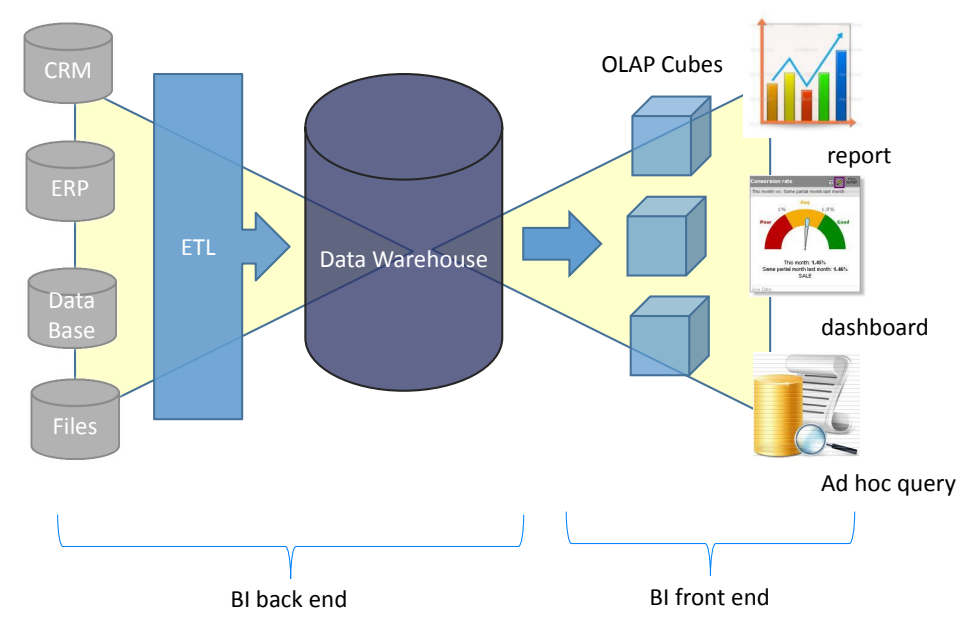

Figure 2.1: business intelligence architecture

their operational systems. These operational systems contain large amounts of transactions that record the day-to-day life of the businesses. Examples of such operational systems might be order-entry systems or stock-control systems. Operational data in these operational systems describes the businesses' specific activities, processes or events. By using Extracting, Transformation and Loading (ETL) tools, BI retrieves operational data and restructures it while loading to facilitate analysis for decision-making. This restructuring can involve data operations such as summarization, aggregation (e.g. sum, average, max, min), or data joins. For example, operational system data may contain sales_quantity and unit_price for each product but in the BI system extra data like sales_amount may be required. The sales_amount based on sales_quantiy and unit_price must be calculated during the load; Another example: operational sales transaction may only contain store_id, while data is transformed to BI, city_id, province_id, region_id may be added. A data warehouse has a separate copy of the data for data analysis, data mining, and reporting activities, so that these operations do not affect the performance of core operational functions of the businesses [6]. Operational activities happen very often, so the data is constantly changing and growing. In contrast, a data warehouse is more static for analysis and updated regularly at certain time intervals. After data has been loaded to data warehouse the OLAP sever 
fetches data from the data warehouses, organizes them in highly complex multidimensional data cubes, and presents it to the users through a user defined format or dashboards [16].

In recent years corporations invested heavily in BI implementations. According to Gartner Report BI and Analytics was a $\$ 12.2$ billion market in 2011 [45]. The big 5 vendors who are SAP, Oracle, SAS, IBM and Microsoft dominated more than $70 \%$ market share in 2011. Those numbers are expected to continue increasing in 2013, 2014, and 2015 and according to IDC the amount will reach $\$ 50.7$ billion in revenue by 2016 [62]. The IBM study of CIO priorities in 2013 also shows Business Intelligence and Analytics is becoming a top technology priority [46].

\subsubsection{OLAP and data cube}

OLAP is one of the key technologies of a business intelligence framework. It is a powerful tool that enables users to quickly and interactively access multiple views of information. For example, users can request reports about all products sales revenues in Europe over a particular year but users can also drill down to the sales of TVs in September, calculate year-to-date sales or compare the revenue with those of the same products sold in January.

OLAP stores data in data cubes models with multiple dimensions. For example, sales by region, sales by sales representative, sales by category, sales by quarter, etc. The OLAP cube consists of dimensions and numeric facts called measures. These dimensions and measures are stored as star or snowflake schema (see figure 2.2) [14]. Measures (e.g. Sales revenue, unit sold, customer count) are derived from the records in the fact table and dimensions (e.g. customer, product, time) are derived from the dimension tables. Normally OLAP cubes have more than 3 dimensions, and each dimension contains several attributes. For examples, a store dimension can have attributes like country, region, or city; a product dimension can have attributes like brand or category or department. Each cell in a cube contains measures which are appropriately aggregated along its dimensions. The aggregation operations can be SUM, AVG, MAX, MIN, etc. Figure 2.3 shows a 3 dimensional data cube with Store, Time, and Product dimensions. The value 56 in the cell represents the total number of units of breads sold in L.A on Monday, so the "unit" is a measure and its value in this case is 56.

The three basic operations provided by OLAP are slice and dice, roll-up, and drill-down 


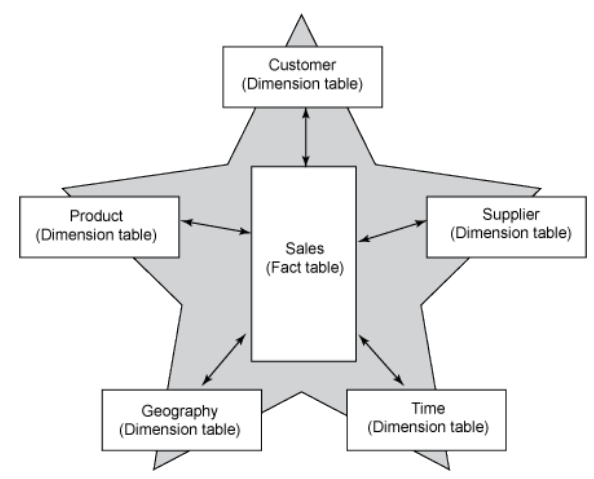

Figure 2.2: Snowflake schema for multidimensional database [14]

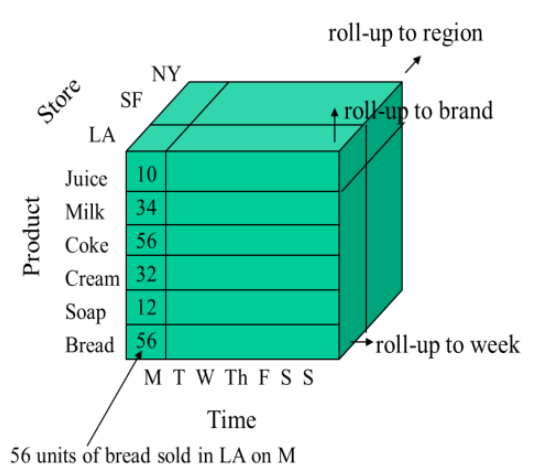

Figure 2.3: a 3-dimensional data cube [42]

[8]. They are defined as follows:

- Slice and Dice:

User can slice the cube and take one specific subset of the OLAP cube on one dimension member, or dice the cube and get the sub-cuboid on two or more dimensions.

For example, in figure 2.4 users are interested in a slice of data corresponding to Asia. User can also dice the cube to analyze the subset of data corresponding to cell phones sold in North America in year 2000 (Figure 2.5).

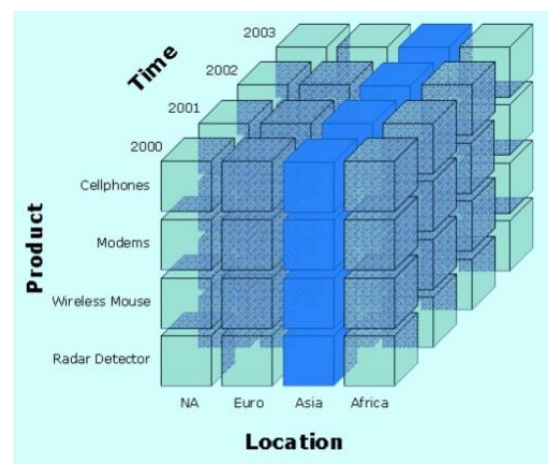

Figure 2.4: a slice of data cube corresponding to location of Aisa [8]

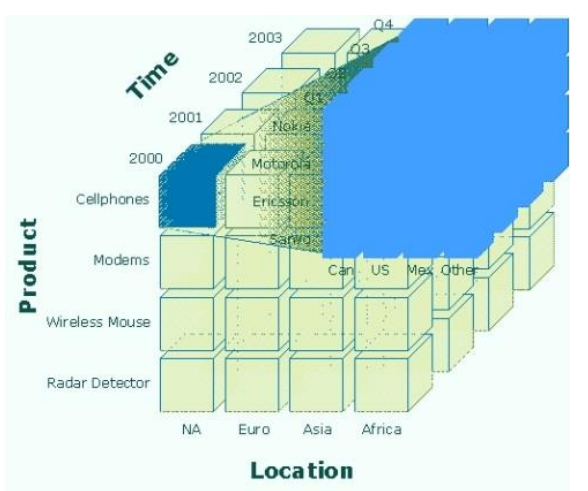

Figure 2.5: a dice of data cube corresponding to cellphones sold at North America in year 2000 [8]

- Drill-down/up:

Users can navigate data from the most summarized to the most detailed levels or the 
other way around from detail levels to more summarized levels. For example: in figure 2.6 users can drill-down and view all product sales at the store EverMore or drill even further down and see the sales for the specific date 2008/04/05. Alternately users can drill-up to the store dimension's "state" and date dimension's "month" to view food products sold at all stores in Florida in April 2008.

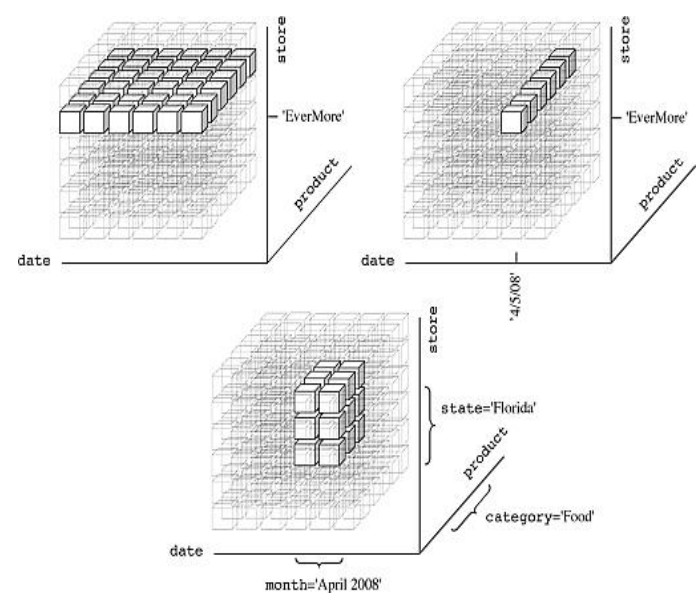

Figure 2.6: drill-down data cube to a specific Store 'EverMore'; (b) drill-down data cube to Store 'EverMore' and date '20080504'; (c) drill-down data cube to Store 'EverMore', month of date 'April 2008' and product category 'Food' [8]

- Roll-up:

A roll-up involves a data aggregation in one or more dimensions. For example, all sales offices are rolled up to the sales departments, or the sales months are rolled up to the years.

In a data cube each cell is a summary value of users' interest and is pre-calculated while the cube is being built. If a user asks for the total sales by year and city, those numbers are already available. This design enables OLAP to response users' queries faster than it otherwise would be able to. For instance, the largest cube in the world is currently 1.4 terabytes and its average response time to any query is 1.2 seconds [35]. This is the big advantage of building a data cube. It has been claimed that OLAP cubes can respond to complex queries in $0.1 \%$ of the time of an OLTP relational database [21]. The multidimensional structure allows users to study data thoroughly to gain comprehensive and valuable business insights. 


\subsubsection{Real-time OLAP}

OLAP systems do not usually run against OLTP systems or other high-transaction, lowlatency databases because analyzing and aggregating on large volumes of historical data consumes extensive time and resources and it can affect the performance of the relational databases. Some businesses, for example financial risk assessment businesses, may require analysis based on current data. Businesses such as these require a Real-Time OLAP (RTOLAP) that performs analysis on integrated data sources in real-time. However, designing a RTOLAP is difficult since the OLTP data model is very different from the OLAP data model. In such a real-time system query performance becomes a concern since values need to be calculated in real-time instead of being retrieved from pre-computed data sets in a traditional OLAP system.

The definition of RTOLAP may vary from business to business. Some researchers define RTOLAP as a system that any data incorporated into a data warehouse should be made available to the OLAP system as soon as possible. In other words, data added to the warehouse should be available to the system immediately so it can be included in analysis and reports. This is the definition for RTOLAP we use in our research.

\subsection{Parallel computing and big data processing}

\subsubsection{Introduction to parallel computing and its advantages}

Parallel computing means executing discrete parts of a computational problem simultaneously on multiple computing resources. Computer resource can be a single computer with multiple processors, many computers in a network or a combination of both. This compares to using sequential computing, executing discrete parts of a problem in sequence on a sin-

gle CPU. The advantage of using parallel computing is to save time and cost. In addition, parallel computing can be used to solve larger and more complex problems such as large scale data processing which is not practical on a single CPU. Parallel computing is not only limited to the local network but can be run on a wide array of resources such as a cluster in a cloud. It provides a more efficient way to solve many difficult science, engineering and business problems. Parallel computing as proven useful for database systems, data mining, and multimedia processing, medical imaging and diagnosis, advanced graphics and virtual 


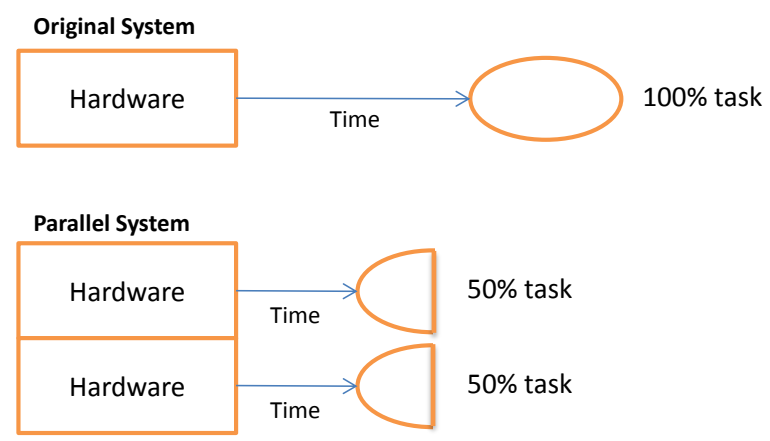

Figure 2.7: Speedup by parallel computing [10]

reality systems etc.

- Parallel computing performance measure: speedup

The goal of parallel computing is to have performance speedup . With the addition of more hardware, the system can complete the same task in less time than the original system [10]. The figure 2.7 shows the speedup. When a single hardware needs an amount of time to complete a task, with parallelism, two sets of the same hardware execute concurrently and only need half time to complete the same task so the speedup is two. In theory, speedup is defined by the formula $\mathrm{S}_{p}=\mathrm{T}_{1} / \mathrm{T}_{p}$ where $\mathrm{p}$ is the number of processors, $\mathrm{T}_{1}$ is the execution time of a sequential algorithm and $\mathrm{T}_{p}$ is the execution time of a parallel algorithm with $\mathrm{p}$ processors.

Ideally, the optimal speedup is linear. That is, when running a parallel algorithm with linear speedup, the speed can be doubled by doubling the number of processors. However, the ideal speedup is hard to reach since it can be affected by the overhead from system synchronization and message passing [50]. Most parallel systems have a near-linear speedup for small numbers of processors and then as more processors added, the speedup performance gains flatten out to a constant value. The diagram (figure 2.8) shows such relationship.

- Parallel processing hardware architectures

Parallel computing architectures can be categorized to the following groups:

\section{Multicore computing}




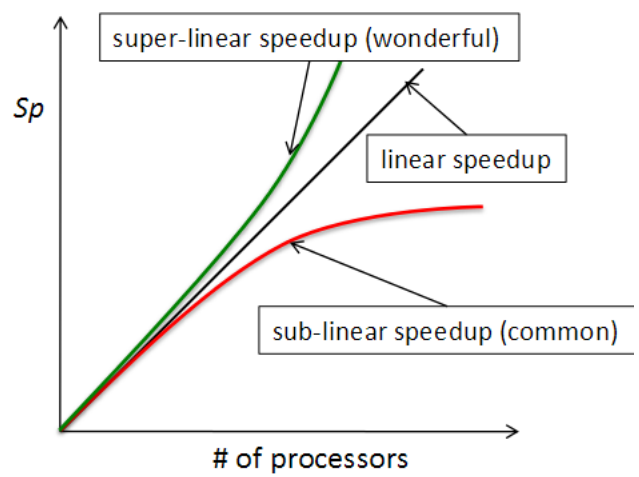

Figure 2.8: linear, super linear and sub linear speedup [63]

A multicore processor is a processor having several central processing units ("cores") on the same chip. Multiple cores can execute multiple instructions at the same time.

Symmetric multiprocessing

A symmetric multiprocessor (SMP) contains multiple homogeneous processors and they share one memory and I/O resource and connect through a bus. Each processor can execute different programs and work on different data.

\section{Distributed computing}

A distributed system consists of multiple processing elements (also called nodes) that connected by network. Each processing element uses its own operating system and memory and they can execute multiple tasks simultaneously $[13,5]$. Each node also acts as an SMP system. The processing elements can be multiple standalone computers. When they are connected by a local network such distributed system can be called a cluster. If the processing elements are multiple processors and they are linked through specialized interconnected hardware, such distributed system is called Massive Parallel Processing (MPP). Typically the number of MPP processors is larger than the members of a cluster. The number of MPP processors can be 100 or even more. Distributed systems can be used in data mining to perform multiple searches of a static database, and they are also useful in artificial intelligence scenarios to analyze multiple alternatives (e.g in a chess playing program). Unlike SMP 
and multi-core systems, distributed systems usually have better scalability, since they can be expanded by simply adding new nodes. Multi-core systems are limited by the number of cores availabe and SMP can be limited to the number of processors.

\subsubsection{Use of parallel computing on large scale of data processing}

In this age of data explosion parallel processing is essential to processing a massive volume of data in a timely manner because it allows data to be divided among the available computing resources and processed independently to achieve performance and scalability [52]. In many areas, such as meteorology, complex physics simulations [50], genomics [52], biological and envioronmental research [17], internet search, financial risk assessment and real-time business analysis data volumes can reach terabytes or petabytes in size. It is difficult to work with data sets of this size since regular relational databases, statistics tools, visualization software products are often not scalable to this level. Parallel processing approaches make working with large data sets possible. For example, decoding the human genome can be achieved in one week with parallel computing where without it it could take 10 years [11]. Parallel computing has become popularized by successful commercial products for instances MapReduce by Google, Hadoop used by Yahoo, Facebook, DryadLINQ by Microsoft and HPCC by LexisNexis etc.

\subsubsection{Parallel computing on cloud platforms}

Parallel data processing is also used for cloud computing. Cloud computing platforms become popular in the past several years, especially among the small businesses. In cloud computing, resources including hardware and software are delivered as a service over a network. Users can easily reserve nodes and set up a virtual machine or a virtual cluster for parallel computing. The emergence of cloud services provides users alternatives to investing in hardware, software and expertise to set up their own cluster. Not only are cloud services cost-effective but also cloud performance is competitive with the performance of in-house clusters [64]. In addition to the flexibility and scalability of using cloud computing, cloud based platforms are a cost-effective solution to run parallel applications, especially data-intensive parallel programs that requires scalability. 


\subsection{Summary}

OLAP usually deals with large scale data, and parallel computing has the advantage of improving system performance. To solve the problems currently existing in OLAP we are applying parallel computing to OLAP systems so that the systems are able to provide better responses to user requests while dealing with large quantities of data. 


\section{Chapter 3}

\section{Review of the State of the Art}

\subsection{Introduction}

We are aiming to design a parallel OLAP system on a distributed cloud platform for realtime analysis. The following is the literature review on related work such as sequential index for OLAP cubes, parallel cube building on parallelism supportive systems and some proposed real-time OLAP systems.

\subsection{Sequential indexes for multi-dimensional databases}

An OLAP cube is a multi-dimensional data array. In relational databases, balanced trees, $\mathrm{B}+$ trees and hash tables are popular data structures to implement indices to optimize data look-up performance. But those indices show poor performance for multi-dimensional range queries. There are some researches on dynamic indexes for multi-dimensional database. In 1984, A. Guttman first proposed R-tree for indexing spatial data [40]. Spatial objects are described by multiple dimensions. The idea of R-trees is to group nearby objects and represent them with minimum bounding rectangles (MBRs) at each level of the tree. Figure 3.1 shows a two-dimensional space with a R-tree index. R8, R9, R10 are the MBRs for the objects D1, D2, and D3 respectively; R3 includes R8, R9 and R10, so it is the MBR for the three objects D1, D2, and D3. Figure 3.2 shows a three-dimensional space with an R-tree index.

Because all objects are contained in a bounding rectangle, a query can be quickly resolved through finding the bounding rectangle overlaps between the query and the objects. When inserting an object into the tree, we should consider finding the node which will have the minimal enlargement of its bounding rectangle. If the capacity of a node is full, the node should be split into two nodes and the rectangles also should be distributed between them. A good split should result in minimal rectangle overlap of these two new sets. 


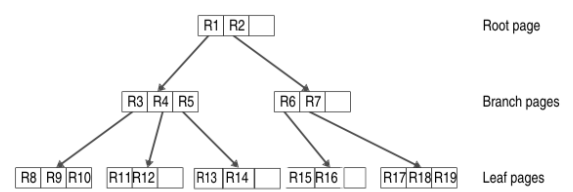

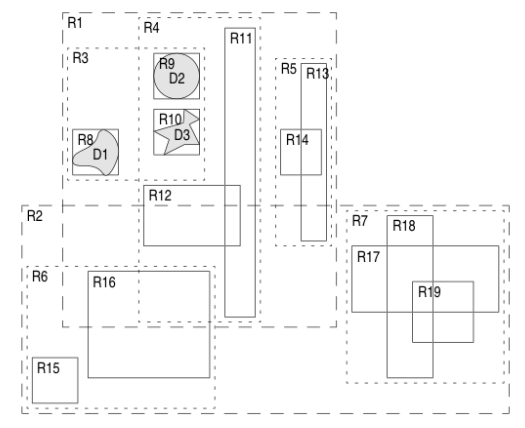

Figure 3.1: an R-tree for 2D rectangles [12]

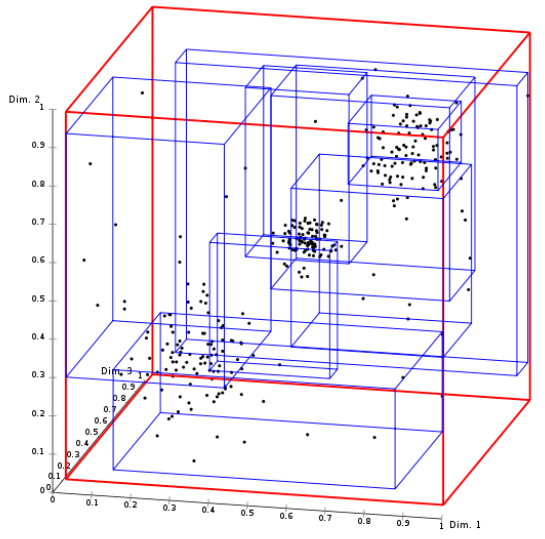

Figure 3.2: Visualization of an R-tree for $3 \mathrm{D}$ cubes [12]

The R-tree is used to index special data with multi-dimension; however, it is not suitable for high dimensional data sets since the overlap of the bounding boxes increases a lot when the dimensions grow. This will cause the search process to be inefficient since it requires accessing large numbers of children nodes. To avoid this problem S. Berchtold et al proposed the $\mathrm{X}$-tree in [18]. The X-tree is based on the R-tree but contains 3 types of nodes: data nodes, normal directory nodes, and supernodes (see figure 3.3). The data node contains minimum bounding rectangles (MBRs) with pointers to the actual data objects. The directory node contains MBRs and pointers to sub-MBRs. Supernodes are directory nodes that contain oversized directory node blocks.

The X-tree can be considered as a hybrid of a linear array-like directory and a hierarchical R-tree-like directory. In other words, the data sets having high overlap are organized in a linear structure and those having not too much overlap can be organized in a hierarchical structure. The most important part is the insertion and X-tree proposed an algorithm to create overlap-free split during an insertion. When the node overfilled and there is no good split, a supernode will be created. The performance comparison between the X-tree and the R-tree proved that the X-tree has consistently better speedup.

A DC-tree, proposed by H. Kriegel in [36], is similar to the X-tree, but the MBR is replaced with an MDS (minimum describing set), so it can be used with high-dimensional 


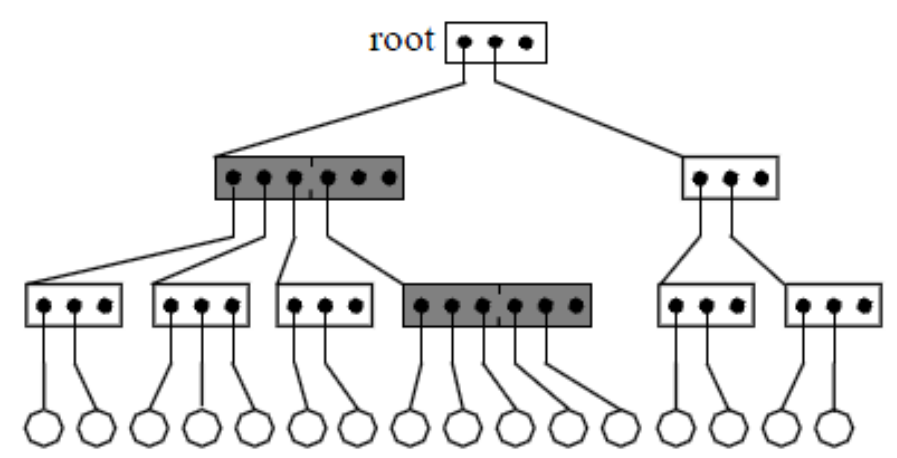

$\square$ Normal Directory Nodes $\square$ Supernodes O Data Nodes

Figure 3.3: Structure of the X-tree

database for OLAP systems. Kriegel states that each dimension of a data cube can be organized in concept hierarchies. Each dimension in a data cube can contain several attributes. These attributes usually can be organized in hierarchical schemas. For example, in the dimension "store", as well as store ids, there can be columns like "country", "province", and "city" which are the attributes describing each store id. The "store" dimension can be organized in a hierarchy as figure 3.4 shows.

We can use an MDS to describe data using its dimensions' conceptual hierarchy values.

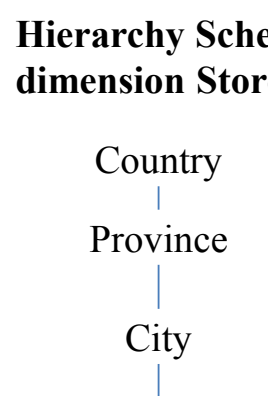

Store ID

\section{Conceptual Hierarchy for dimension Store:}

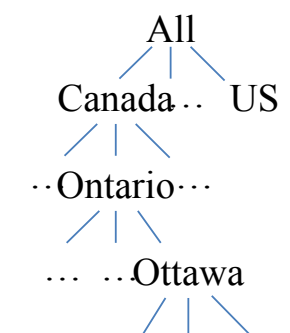

...3001 ...

Figure 3.4: Hierarchy Schema and Concept Hierarchy for dimension Customer 
An MDS contains multiple sets of attribute values at different hierarchical levels which describe cube dimensions. Consider the following two data records in a data cube having 3 dimensions: "Store", "Product", "Time" and one measure: "Sales".

([Canada, Ontario, Ottawa, 3001], [Food, Bakery, 225], [2013, March, 30, 123], $\$ 2703.89)$

([Canada, Quebec, Montreal, 4001], [Food, Candy, 625], [2013, Feb, 20, 456], \$6703.89)

The MDS at the lowest level in the concept hierarchy of each dimension is $(\{3001$, $4001\},\{225,625\},\{123,456\})$. Since the attributes id at the lowest level of a hierarchy usually is a surrogate key in each dimension, an MDS at the lowest level can distinguish each data record from each other. However, an MDS can also use values of higher levels in a concept hierarchy. For the above same data records, the MDS can be ( $\{$ Ontario, Quebec $\},\{$ Food $\},\{2013\})$. The values in each MDS set for a dimension should all belong to the same level in the concept hierarch of this dimension. For example, for the "store" dimension, it is correct to use \{Ontario, Quebec $\}$ as the MDS, but not Ontario, Canada. Similar to the bounding box in the R-tree, the MDS describes the boundary of data records. The attribute values in a hierarchy also have the property of partial ordering which allows easy insertion and range queries since the leaf of the concept hierarchy is organized as a group.

H. Kriegel's paper also proposes the algorithms for insertion and range query operations. The insertion traces down from the root of the tree by comparing the MDSs of directory nodes with the MDSs of new data. When the node capacity is reached, the node will be split. Before performing the hierarchy split, the MDSs of the entries and the MDSs of directory nodes have to be adjusted to the same hierarchy level in order to calculate the MDSs' enlargement and overlaps. It always inserts the node to the group having the minimum MDSs overlaps with others. The range query algorithm finds the MDSs of directory nodes which contain the range query's MDSs and adds the measure to the result. $\mathrm{H}$. Kriegel's paper compared the performance of the DC-tree, the X-tree, and the sequential search algorithm. The insertion time is significantly lower for the X-tree since the DC-tree has a higher computational load than the X-tree has. However, the DC-tree provided better performance with range queries especially when the range query's MDSs were large. The DC-trees are designed specially for OLAP multi-dimensional databases enabling users to 
execute data insertions and search queries sequentially.

\subsection{Buiding data cubes in parallel systems}

Many parallel algorithms are developed to support multi-dimensional data cubes in order to reduce the query response time. F. Dehne et al. proposed several methods to build static data cubes on cluster platforms in papers [23, 27, 28, 29, 30, 31, 32]. The main idea of $F$. Dehne et al's methods is to provide data partition strategies to assign sub-cubes to different cluster nodes and then to build sub-cubes on each node. S. Goil et al presented parallel algorithms to construct data cubes using sort-based and hash-based methods [38]. The experiments of S. Goil et al's methods showed good performance on small data sets. H.Luan et al. [47] proposed an approach called Cache-conscious Cubing (CC-Cubing) which can partition multi-dimension data simultaneously with multi-threads to enhance CPU and cache performances. H.Luan et al. claimed that the parallel cash-aware CC-Cubing algorithm improved the performance by $27 \%$ comparing it to the single thread CC-Cubing method.

D.Jin et al. [43] implemented a parallel algorithm to build data cubes based on dynamically extendible multi-dimensional arrays using the multi-core architectures. The extendible array can be dynamically extended along any dimension without relocating any existing data. The experiments were performed on a SUN Enterprise 4900 SMP machine with 24 cores processors and obtained near linear speed up and showed good scalability for high dimensional data sets. These parallel algorithms are focused on building static data cubes for a better query performance.

\subsection{Real-time OLAP systems on cloud architectures}

With the emergence of cloud services, many applications are migrated to cloud infrastructures. The strategies and methods for hosting BI applications on cloud architectures is still being researched. BI is a highly resource intensive system requiring large scale parallel processing and significant storage capacities to host the data warehouses. With all the advantages of cloud services such as scalability or no maintenance on hardware infrastructure, cloud is an alternative platform for BI systems. 
H. Al-Aqrabi et al. in [16] designed a small BI and OLAP framework model with massive parallel relational database servers to demonstrate that a OLAP system can be taken to cloud hosting. However, the model was built on a simulation environment limited only to certain ideal scenarios, for example, query loads were evenly distributed. In the real practice, it is challenge to evenly distribute loads when the systems involve hundreds of databases. [65] proposed a R-tree and KD-tree combined index structure to improve cube building and query performance. [20] presented $E S^{2}$, an elastic data storage system in a cloud environment to support both OLAP and OLTP within the same storage. They developed a unified indexing framework including hash indices for exact queries and distributed multi-dimensional indices for multi-dimensional queries. The experiments of $E S^{2}$ illustrated the system efficiency and confirmed the benefit of providing distributed indexes and accesses to the data for both OLTP and OLAP queries. However, no benchmark has been provided yet.

Both MapReduce and Hadoop support to process large data sets especially unstructured data in a distributed system which can be scaled up to thousands of machines. Several high level OLAP models have been built on distributed systems using a MapReduce programming model or a Hadoop framework to provide data processing and analysis. Paper [34] proposed to convert multidimensional data structures into Pig [53] data and allow using Pig Latin query language to perform OLAP search. Hive [60] is also a data warehouse solution built on a Hadoop framework. It stores data in tables similar to relational databases and supports queries using SQL-like language called HiveQL. It has been used by Facebook for data analysis. epiC is an elastic power-aware data-intensive cloud platform for data analysis operations and online transactions [22]. These studies focused on operations with relational data models using the data-intensive computing technologies. Paper [19] proposed to build a distributed cube with a broker architecture on Amazon EC2. This system is able to store, manage, analyze data and answer OLAP queries in a fully elastic manner. When the cube storage is increasing, it can be extended to a virtual cube where it can host more regular cubes. The implementation demonstrated near linear speedup for OLAP query response time while increasing the number of cubes. U. Ahmed et al in [15] proposed a real-time temporal OLAP with the TiC-Tree. They redefined the MDS and take advantage of the temporal nature of the data in the dimension of "Time" to keep values 
together in time order in the tree nodes. This model provided better group-by and range queries performance. 


\section{Chapter 4}

\section{Problem Statement}

\subsection{Introduction}

With the increasing demands on performing data analysis in real-time on large scale data, more and more researchers pay attentions to improve the performance BI or OLAP. Due to the change of businesses requirements, OLAP systems encounter some challenges. 1) Business data is growing rapidly so the existing OLAP may be lack of storage space soon. It requires adding more infrastructures with large memory for computing data and large disks for storing data. 2) OLAP is not optimized well for handling very large data sets, so it cannot response user requests quickly enough. But, many businesses such as stock exchange businesses require high efficient performance. 3) Many online services are emerged and they also require business analysis in real-time. However, the OLAP systems in a periodical update fashion cannot provide the most up-to-date data for business analysis. Therefore, users are looking for solutions that enable OLAP systems to include the new data once they are available to business intelligence systems instead of doing periodical loading.

In the reviews in Chapter 3, various possible solutions have been discussed and some of them even have been implemented as real products. They are built on different data models, system architectures, and hardware platforms to achieve optimized data analysis performance in different ways. To meet the above requirements, we are aiming to design a real-time OLAP system which can execute new data insertions and OLAP operations (e.g. drill-down, slice, roll-up) concurrently. The system can run on distributed systems such as a cluster on a cloud environment so that it can be scale up to handle the growth of data volume.

Next, we will describe the details of the problems and requirements being concerned in our study. 


\subsection{Problem statement and justification}

\subsubsection{OLAP data size is increasing dramatically}

An OLAP system is designed to provide quick responses to user query requests for the purpose of business analysis. Usually, an OLAP system contains historical data loaded from transaction systems as well as aggregation data pre-computed during data loading. Over time, the data gets exponentially increased. As we discussed in Background Chapter, the data size can be in terabytes or even petabytes. This also requires much larger data storages. Therefore, while the data is growing, the OLAP will require additional storages to maintain all data. Adding extra infrastructures on existing systems can be costly and merging the existing system into the new infrastructures can be also difficult sometimes. If a system allows to be easily expanded, it could not only save large amount investments but also avoid troubles caused by technical problems.

Furthermore, the technologies to handle large data sets will be different from the ones to handle small data sets. The MapReduce programming model and the Hadoop framework are developed to process big data across distributed systems. But they are mostly used for unstructured data for data mining purpose. Several frameworks are suggested to deal with large volumes of data, for examples, Hive, Pig, and epiC [60, 22, 53]. These systems use the MapReduce programming model and execute OLAP queries in relational databases instead of multi-dimensional databases. An elastic OLAP on cloud was proposed in [19] to efficiently utilize cloud computing infrastructures to provide scalability. But it was using its own representation language called OLAP Model Markup Language (OMML) for data cubes, OLAP queries and analytical results.

\subsubsection{Query performance is critical}

The large data involved in OLAP systems can also hugely impact the performance of OLAP systems. Organizations use OLAP systems to quickly response the market changes, therefore, response time of OLAP operations and ad-hoc queries is critical for most users. Most OLAP engines have already been optimized for the purpose of business analysis so that they can report on millions of records at a time. However, query efficiency is still a concern 
in OLAP systems while they involve large scale data sets. User queries usually are various so aggregations for certain types of queries may not be pre-calculated in data cubes. Queries against the large scale data sets can be extensively time consuming since they can access billions of data rows and require a lot of data scans, joins and aggregates.

- Using data indexes to optimize multi-dimensional databases operations

The OLAP data is represented in multi-dimensional database. Many cube may contain 20,30 or even more dimensions. In most commercial relational database management systems (RDBMS) such as IBM DB2, Informix, Microsoft SQL Server, Oracle 8, Sybase ASE and SQLite, $\mathrm{A} \mathrm{B}^{+}$-tree is implemented for a table index in order to achieve optimized query performance. This type of tree is designed for searching data according to one attribute so it is not efficient to answer queries concerning multiple attributes. For a multi-dimensional index structure, an R-tree was considered as a good solution [40]. Oracle and DB2 have the extensions that are supporting spatial indexing with the R-trees, but they are mostly used for GIS/CAD applications.

However, when the number of dimensions is higher than 5 to 10 , the R-tree and its variants like $\mathrm{R}^{+}$-tree, $\mathrm{R}^{*}$-tree are not efficient any more [41]. MBRs in high dimensions could have huge overlaps so it needs to traverse many nodes in the tree to answer a query. The $\mathrm{X}$-tree is also an extension of the R-tree and it is designed for high-dimensional databases but it is also used for spatial objects [18]. The DC-tree is a dynamic index specially designed for data warehouses and its structure allows to handle insertions and range queries sequentially [36].

- Constructing data cubes for parallel queries

With the emergence of multi-core processors, parallelization becomes a popular ongoing research and an optional technology to be adopted by developing business intelligence applications. The R-tree was parallelized to improve the performance on multi-dimensional data $[59,56,25]$, however the focus of these discussions is still on spatial data but not for the design of OLAP multi-dimensional data structures. Some researchers have worked on parallelizing OLAP cube computing. [38, 39] discussed parallel cube computing for multi-dimensional OLAP using in-memory data cube construction on distributed-memory machines. H. Gao et al in [37] proposed parallel 
hierarchical cubes that support parallelizing range sum queries but their method was hard to be applied to other type of aggregations. The ParaCube introduced in [66] used a mediator to distribute queries to all sibling cubes and merge all sub queries' results to a global result. But the authors stated that the system performance was influenced by the conflicts on accessing shared memory when applying such a structure on multi-core platforms.

\subsubsection{Data freshness is demanded}

Some businesses(e.g online shopping or real-time traffic surveillance) have very high demands on data analysis in real-time. They would like to have any data added in OLTP be available to business intelligence systems right away and to be included in reports. The OLAP data are usually imported from external OLTP systems. In the process of loading, it involves large computation on the raw data in order to transform them to a multidimensional model. OLAP systems usually get updated periodically because data precomputation could take very long time to complete while building data cubes. Therefore, most OLAP systems are just an information snapshot at one point in time. The data cubes are not dynamically built. Consequently, the systems are not in real-time and the data used for decision support are possibly out-of-date which can lead to ineffective decision making. These OLAP systems are less valuable for those businesses who need most up to date information. [15] designed TiC-Tree for a real-time OLAP, but it is primarily used on temporal data. $[57,58]$ proposed a real-time data warehouse which enabled continuous data integration through an adaptive loading methodology to minimize the impact of OLAP queries. However, this approach focused on incremental data loading to data warehouses and building static OLAP cubes. Jones in [44] stated one problem of using OLAP systems for data analysis in real-time is reformulation of multi-dimensional data cubes. Some researchers also suggested using XML to map OLTP and OLAP to achieve analysis in real-time [24].

In addition, a discussion has been made on the topic of integration of OLTP and OLAP to provide real-time analysis [24]. The author reviewed several related technologies and studied the possibility to use the same database engines for both systems with the object extensions of the SQL3 standard. He also suggested four major approaches to constructing Real-Time Data Warehousing (RTDW) but no performance evaluations on an implemented 
system were provided.

\subsection{Evaluation of Results}

In order to resolve the above issues, we intended to design a parallel OLAP system for realtime analysis on a distributed system with cloud infrastructures. We developed a method to partition data and distribute them to the available computing processors in the system. It enables dispatching requests such as data insertions and range queries to the related nodes so the requests are executed in parallel. The system provides analysis in real-time since it will include most recent data in the results for query requests. To evaluate the performance of our system, "speedup" is the key measurement we used. We expected that the system should achieve near-linear speedup by increasing the number of nodes in a distributed system.

\subsection{Summary}

In this Chapter, we presented the three main concerns in OLAP systems, such as the system scalability for increasing data size, the response performance for query requests and the data freshness for real-time requirements. Though there are some studies or commercial products available, most of them do not provide complete solutions to all the problems we listed in this chapter. Therefore, we aimed to design an enhanced parallel system to resolve these issues. 


\section{Chapter 5}

\section{Parallel real-time OLAP on multi-core processors}

\subsection{Introduction}

In this Chapter, we will introduce a parallel real-time OLAP system for multi-core processors. It is designed by F. Dehne and H. Zaboli based on the sequential DC-Tree. The tree data structure provided in the parallel real-time OLAP is called Parallel DC-Tree (PDCTree). It enables concurrently executing multiple insertions and multiple range queries on an architecture with multi-core processors [33]. It is claimed to be the first fully real-time OLAP system that has been parallelized and optimized for contemporary multi-core processors. The evaluation was performed on the platforms with a maximum 20 cores and it shows the system can achieve near linear speedup by increasing the number of cores.

The PDC-tree also provides the foundation for designing a parallel real-time OLAP for distributed systems in a cloud environment. To evaluate the performance of the PDC-tree in a cloud environment, I conducted various experiments on the Amazon cloud EC2 platform as well as the Ontario Research and Education VCL cloud.

In next section, we will review the data structure of the PDC-Tree and present the results from the experiments I performed in the cloud environments.

\subsection{Parallel DC-Tree for multi-core processors}

The parallel real-time OLAP system introduced in [33] takes the idea of hierarchy structures for multidimensional databases and the directory node structures introduced in the DC-tree. In addition to the DC-tree data structure, the PDC-tree index consists of two additional parts which are 1) time stamp 2) link to sibling (See figure 5.1 and figure 5.2). The time stamp stores the most recent time when the directory nodes in the tree are modified or created during the process of a node splitting or a node insertion. The "link to sibling" is used to maintain a linear chain between the children of each directory node. These two 
fields are important for the system to perform parallel executions. Also the design acquires the lock to only lock the nodes which are currently being updated. The authors provided

\begin{tabular}{|c|c|c|c|c|}
\hline MDS & TS & $\mathrm{M}$ & $\begin{array}{c}\text { Child } C \\
\text { Link }_{1} \\
\text { MDS }_{1} \\
\mathrm{TS}_{1}\end{array}$ & $\ldots$ \\
\hline
\end{tabular}

Figure 5.1: data structure of a directory node in PDC-tree

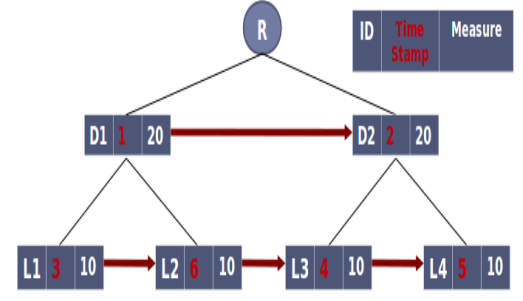

Figure 5.2: the structure of PDC-tree

the following algorithms of data insertion and range query operations.

The PARALLEL_OLAP_INSERT algorithm tracks down the PDC-tree by checking the MDS in each directory node to find the leaf directory node where the new data item should be inserted. Three cases will be considered.

- When the new data's MDS is contained in only one directory node's MDS, the algorithm traces down its child directory node.

- When the new data's MDS is included in more than one directory node's MDS, the algorithm chooses the smallest subtree to go down.

- When the new data's MDS has overlap with many directory nodes' MDSs, choose the directory node that has minimal MDS overlap with its neighbors caused by the MDS enlargement if possible insertion happens.

This step is repeated until the leaf directory node is reached. Then the node will be locked in order to perform data node insertion. If the node capacity has been exceeded with the insertion, it will call the SPLIT algorithm of node splitting. The figure 5.3 (a) demonstrates a node split during a insertion. The directory node D1 is required to be split. A new directory node D4 is created and added as D1's right sibling node. Meantime D4 copies D1's old timestamp. The children nodes D2 and D3 are distributed between D1 and D4 so that they have minimal overlap between two groups. After split, D1 updates its timestamp with new time and also updates its MDS and measures. In this process, the 


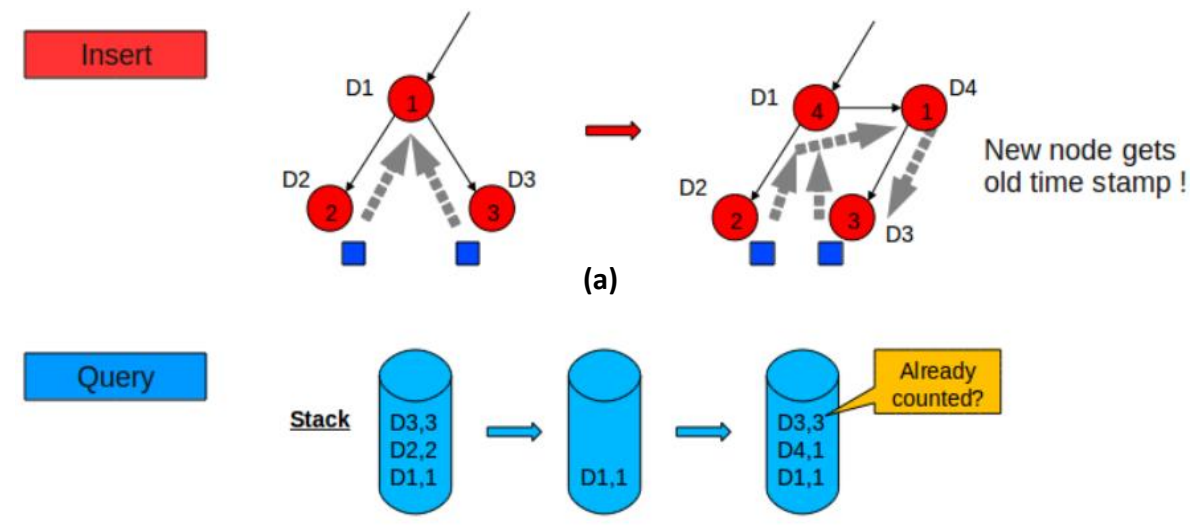

(b)

Figure 5.3: (a) Node split during insertion (b) stacks used for traversing directory node for query [26]

newly added directory node gets the old time and the original directory node gets the new time. The split process starts from the nodes at the bottom of the tree until no further split or the root node is reached.

The PARALLEL_OLAP_QUERY algorithm takes the MDS of the given range query as an input. A stack is used to control traversing the tree and to detect the modified directory nodes and new directory nodes raised by parallel insertions so that all the new data inserted in parallel along with query requests can be evaluated as well. Starting from the root the process pushes one directory node to the stack and then evaluates its children nodes. While comparing the MDS of every child directory node and the MDS of the given range query, the MDSs need to be converted to be at the same hierarchy level for each dimension. The MDS in lower hierarchy levels will be converted to an MDS in higher hierarchy levels. Two cases can happen in this step.

- If the child directory node's MDS is contained in the range query's MDS, then the child's measure is added to the results.

- If the child node's MDS has overlap with the query MDS, then the child will be pushed to the stack.

During the time of searching in a subtree, a directory node A of this subtree could be modified with a new timestamp if a parallel insertion happens. So when A's corresponded 
original directory node $\mathrm{A}_{\text {stack }}$ with earlier timestamp is popped up from the stack, we know a new data node has been inserted into the substree rooted at A or its siblings' subtrees if node splits are performed. The directory node $\mathrm{A}$ in the tree and its sibling nodes need to be evaluated and their subtrees should be re-visited. Therefore, the algorithm traces node A's right sibling directory nodes along the right sibling links and pushes any right sibling directory nodes whose timestamp is later than or equal to the timestamp of node $\mathrm{A}_{\text {stack }}$ in the stack as well as the modified directory node A. In figure 5.3 (b), when a query traverses the tree, D1 and its children are pushed into the stack. When evaluating D2 and D3, D1 gets split due to a new insertion. Now D1's timestamp has been updated. When D1 in the stack is popped up and compared with D1 in the current tree, timestamp of D1 in the stack has earlier time than the timestamp of D1 in the current tree has, therefore, we know a new data has been inserted. D1, D1's right sibling D4 and D4's child D3 will be pushed into stack for revisiting so that the newly inserted data can be visited and evaluated. D2, the child of D1, will be also revisited when D1 in the stack is popped from the stack for evaluation again. The steps are repeated until the stack is empty.

The PDC-tree is the extended version of the sequential DC-tree. Timestamp and Link to sibling directory nodes are added in the data structure of the PDC-tree in order to detect any newly inserted data. The system acquires a minimal locking schema to execute data insertions and queries in parallel. At most two nodes (current node in process and its parent node) are locked at any point in time to perform insertions or updates. The experiments of this method were performed on machines having up to 20-core processors. The paper provided the results and demonstrated significant performance improvement on executing insertions and range queries in parallel. By increasing the number of cores, the system can achieve close to linear speedup. It has the potential to enable the large scale OLAP to become a real-time system.

\subsection{The performance experiments of the PDC-tree on cloud platforms}

The Parallel DC-tree was implemented in C++ with OpenMP by Hamed Zoboli. In order to further evaluate the performance of this Parallel DC-tree, I conducted many tests by changing the values of system parameters, for examples, the number of insertions, queries, threads, the size of initial data rows as well as the children capacity of a directory node. 
The purpose of those tests is to observe the impact of these parameters on the tree behavior and to evaluate the performance of the PDC-tree on cloud frameworks. All the tests are performed on different instances provided by the Amazon web services (AWS) and the Ontario Research and Education VCL cloud (VCL).

\subsubsection{Experiments environment and scope}

- Amazon Elastic Compute cloud

An Amazon Elastic Compute cloud (EC2) is one of the Amazon Web Service (AWS) [3]. EC2 allows users to rent virtual computers (also called instances) to run their own computer applications on. Users can create an EC2 instance with an Amazon Machine Image (AMI). Amazon provides users fully control of their instances. Through web service APIs or accessing the AWS management console, users can boot, stop, resume and terminate any instance as they like. Users also have the root access and can install any software as they want. Amazon provides variety types of instances, operating systems as well as software packages. Several types of EC2 instances were selected to run our experiments on. Table 5.1 is the hardware description of the multi-core instances used in the tests.

M1 and M3 are the standard instances. They are suitable for most general purpose applications; M3 instances have higher CPU performance which are suited better for CPU intensive applications; High-Memory M2 instances contain larger memory sizes for memory-intensive applications such as databases and memory caching applications; High-CPU C1 instances have proportionally more CPU resources than RAM and are well suited for scale out compute-intensive applications. Amazon uses an EC2 Compute Unit (ECU) to measure the CPU capacity. One ECU provides the equivalent CPU capacity of a 1.0-1.2 GHz 2007 Opteron or 2007 Xeon processor [9].

I selected the Ubuntu 12.04 LTS version for the operating system. The source code and the data set were loaded to the cloud machines. I created an AMI of this environment and it was used on every instance for the tests. This provided the same environment for each experiment and limited any unexpected noise from the system and data themselves. 
Table 5.1: Specifications of Amazon cloud instances used in our experiments [2]

\begin{tabular}{lllll}
\hline \hline Instance Name & Memory(GB) & vCPU & ECU & ECU/Core \\
\hline $\begin{array}{l}\text { M1 Large Instance } \\
\text { (m1.large) }\end{array}$ & 7.5 & 2 & 4 & 2 \\
$\begin{array}{l}\text { M1 Extra Large Instance } \\
\text { (m1.xlarge) }\end{array}$ & 15 & 4 & 8 & 2 \\
$\begin{array}{l}\text { M3 Extra Large Instance } \\
\text { (m3.xlarge) }\end{array}$ & 15 & 4 & 13 & 3.25 \\
$\begin{array}{l}\text { M3 Double Extra Large Instance } \\
\text { (m3.2xlarge) }\end{array}$ & 30 & 8 & 26 & 3.25 \\
$\begin{array}{l}\text { High-Memory Extra Large } \\
\begin{array}{l}\text { Instance (m2.xlarge) } \\
\text { High-Memory Double Extra }\end{array}\end{array}$ & 17 & 2 & 6.5 & 3.25 \\
$\begin{array}{l}\text { Large Instance (m2.2xlarge) } \\
\text { High-Memory Quadruple Extra }\end{array}$ & 68 & 4 & 13 & 3.25 \\
$\begin{array}{l}\text { Large Instance (m2.4xlarge) } \\
\text { High-CPU Extra Large Instance } \\
\text { (c1.xlarge) }\end{array}$ & 7 & 8 & 26 & 3.25 \\
\hline
\end{tabular}

Table 5.2: VCL cloud Instances

\begin{tabular}{lcc}
\hline \hline Instance Name & Memory(GB) & Cores \\
\hline HPC CentOS 6.3 & 32 & 16 \\
Generic Mapping Tools & 8 & 2 \\
\hline
\end{tabular}

- Ontario Research and Education VCL cloud

The other environment is the Ontario Research and Education VCL cloud. This is a cloud environment sponsored by IBM and is used for the research and education purpose for the four universities in Ottawa. There are total seven pre-configured systems and we selected the High Performance Compute (HPC) Centos 6.3 with 16 virtual cores for the experiments and the Generic Mapping Tools API with 2 virtual cores. Each virtual CPU speed is at $2.19 \mathrm{GHz}$. See Table 5.2.

- Data Set

We used the TPC-DS benchmark, a decision support benchmark standard from Transaction Processing Performance Council. It includes data schema, data population and 

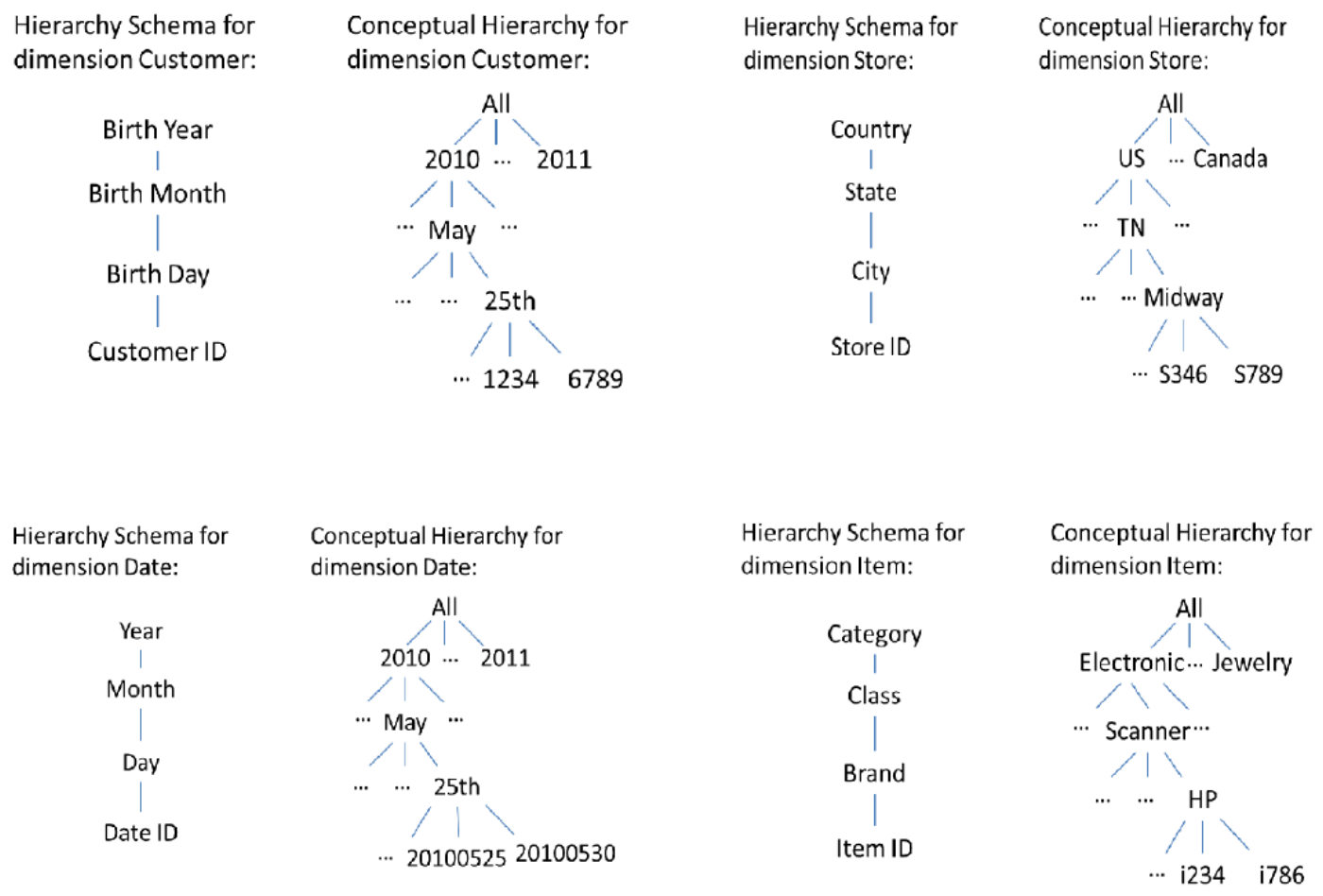

Figure 5.4: Hierarchy shema and Conceptural Hierarchy for each dimension

queries etc. Though the underlying business model of this benchmark is a retail product supplier, it is designed to represent a modern decision support system [95]. The benchmark can be used to exam large volumes of data. The data generator can create $1 \mathrm{~GB}, 100 \mathrm{~GB}, 300 \mathrm{~GB}, 1 \mathrm{~TB}, 2 \mathrm{~TB}$ data sets up to $100 \mathrm{~TB}$. We selected $1 \mathrm{~GB}$ data set as testing data. Not all data tables in the data set are used for our experiments. We use the data from a fact table Store_Sales as insertion data. Four dimensions: Store, Item, Customer, Date_Dim are selected to build the PDC-tree based on the hierarchy schema defined in each dimension. The figure 5.4 shows the dimensions and their hierarchy schemas. The measure is ss_net_profit. The code randomly generates range queries which contain a set of MDSs and have output results covering 5\% or 25\% of total data set. The system initially reads partial data from the fact table to build the initial PDC-Tree and then a mix of insertions and queries operations are sent to the system in sequence.

- Experiments scenarios 
1) Increasing the number of threads

2) Increasing the number of insertions

3) Increasing the size of the initial PDC-tree

4) Increasing the number of the capacity of a directory node for a fixed size of an initial PDC-tree

5) Increasing the number of the capacity of a directory node for a increasing size of an initial PDC-tree

\subsubsection{Performance evaluation}

- Test1: Initial tree contains 10,000 rows of data, 1000 queries, 200 insertions, 25\% output coverage, increasing threads from 1 to 32 .

In this experiment, we tested the query performance by increasing the number of threads from 1 to 32 . In figure 5.5, 5.6, 5.7, 5.8, 5.9, the results are showing that when we increase the number of cores, the total query time will be reduced by average $43 \%$. In order words, the PDC-tree could achieve about $43 \%$ linear speedup. When the number of threads exceeds the number of cores, the speedup is not so obvious. We also observe that the performance of some types of machines is better than the performance of other types of machines is. For example, the total query time achieved by the system on VCL HPC machine is higher than the query time achieved on the Amazon cloud machines. But the system on HPC of the VCL achieves a bit better linear speedup than it does on machines of the Amazon cloud .

In figure 5.7, we notice the system only has $21 \%$ speedup on the m3.xlarge instance while changing the execution thread from 1 to 2 . We suspect that this is associated with ECUs since the processor contains virtual cores not the actual physical cores. The underlying physical cores can be shared with other users, so it slows down the computing. The same test was ran for a few times on the machine m3.2xlarge and we found the results were fluctuating. The total response time for 1000 queries and 200 insertions with a single thread is about 32 seconds. However the response time using 2 threads could be fluctuating from 17 seconds to 25 seconds. 

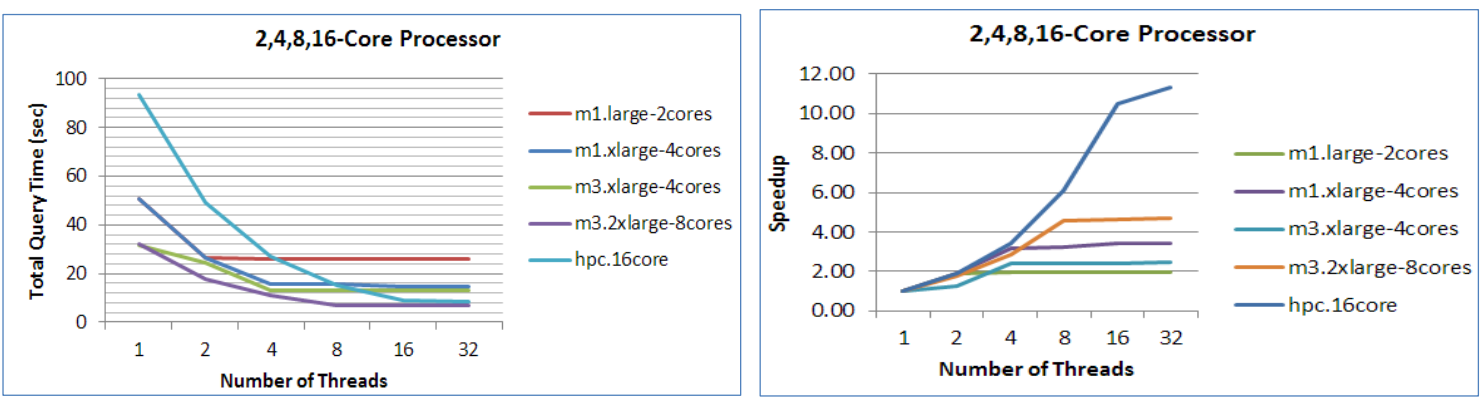

Figure 5.5: Increasing the number of threads on different types of processors
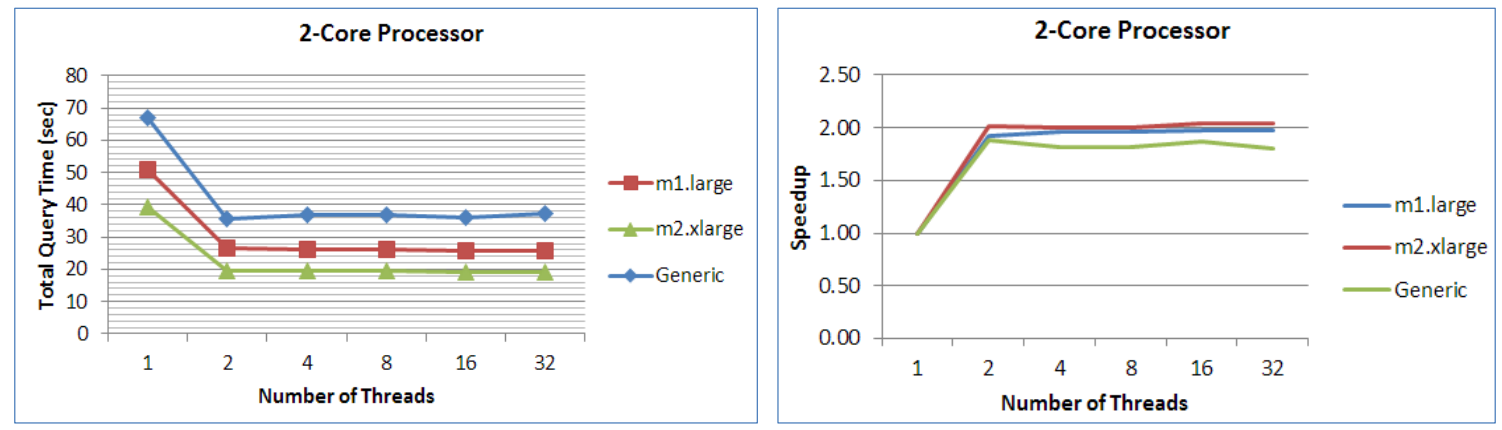

Figure 5.6: Increasing the number of threads on a 2-core processor
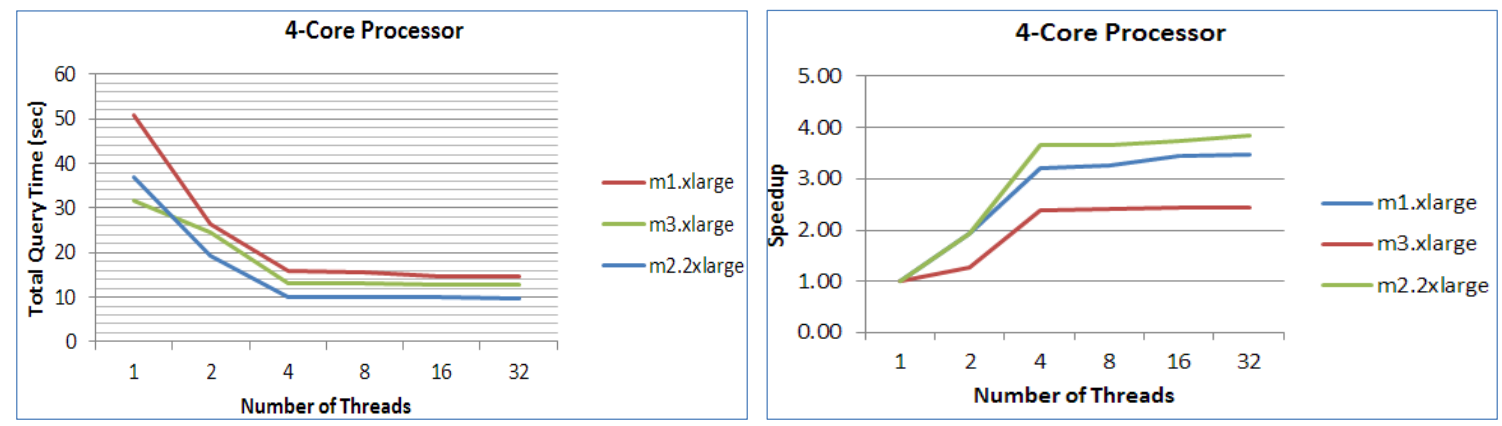

Figure 5.7: Increasing the number of threads on a 4-cores processor 

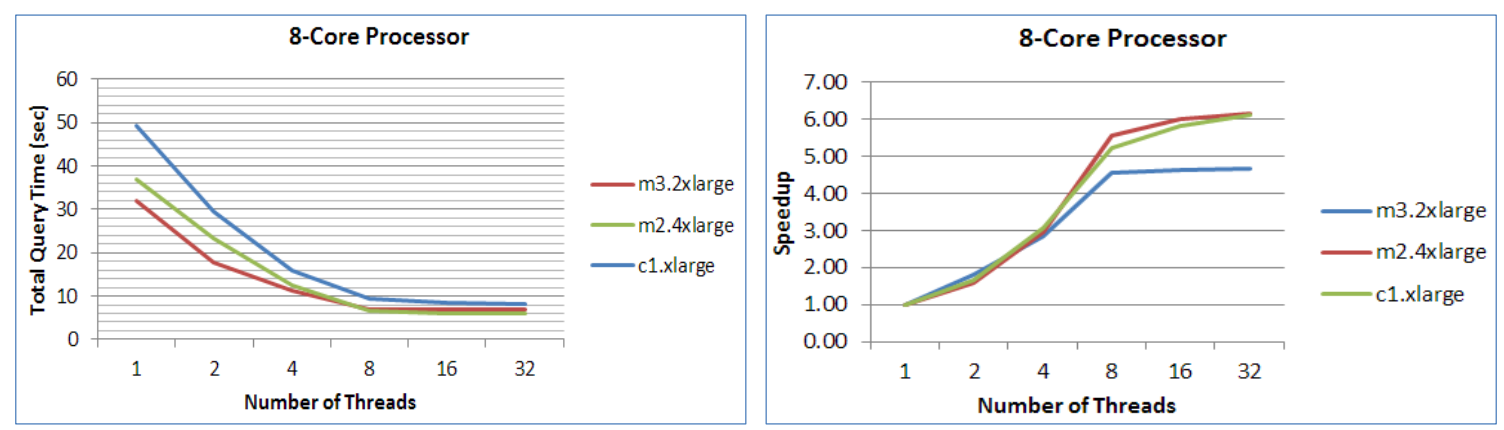

Figure 5.8: Increasing the number of threads on a 8-core processor
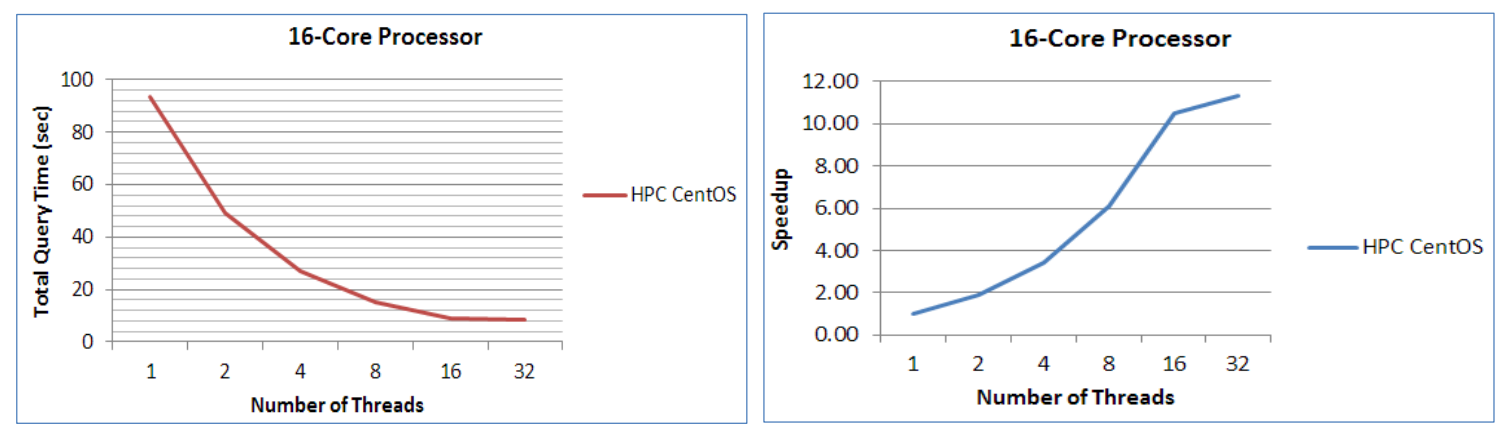

Figure 5.9: Increasing the number of threads on a 16-core processor

- Test2: Initial tree contains 10,000 rows data, 1000 queries with $25 \%$ output coverage for $0,200,1000$ insertions on an m2.4xlarge instance.

In this experiment, we tested the query performance by increasing the number of insertions from 0 to 1000 . We build the initial PDC-tree with 10000 rows then we perform 0, 200, and 1000 insertions. Figure 5.10 shows there is only a little time increasing when the number of insertions is increased. This proves that the minimal locking during process does not cost too much time for all the parallel executions.

- Test3: 1000 queries and 200 insertions with $25 \%$ on an m3.2xlarge machine for different size of the initial tree.

In this experiment, we tested that query performance while increasing the initial tree size. We built several initial trees using 5000 (5k), 10000 (10k), 20000 (20k), and 

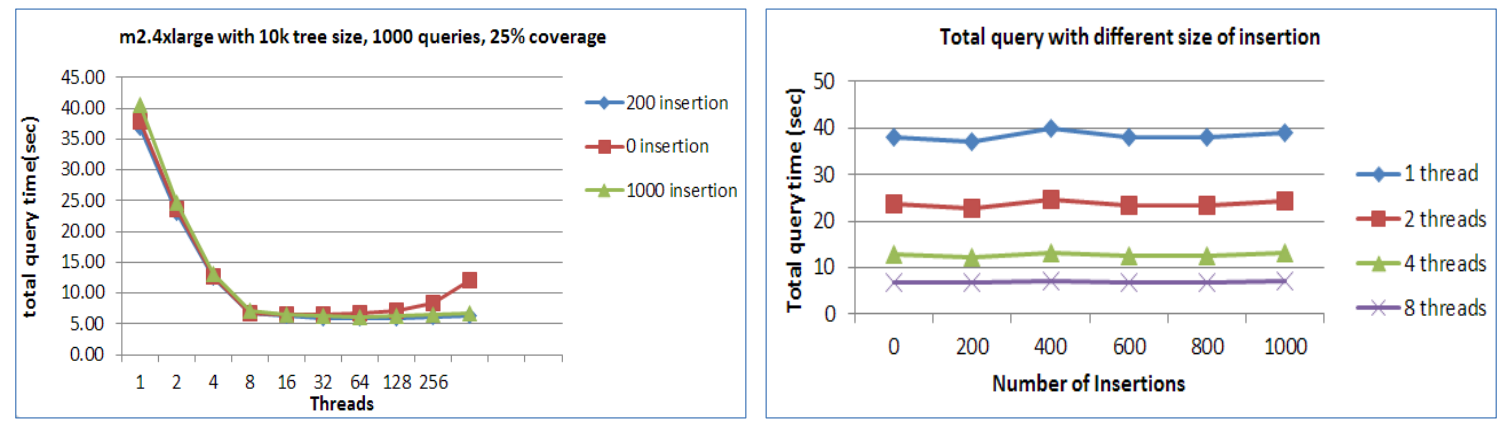

Figure 5.10: Increasing the number of insertions from 0 to 1000
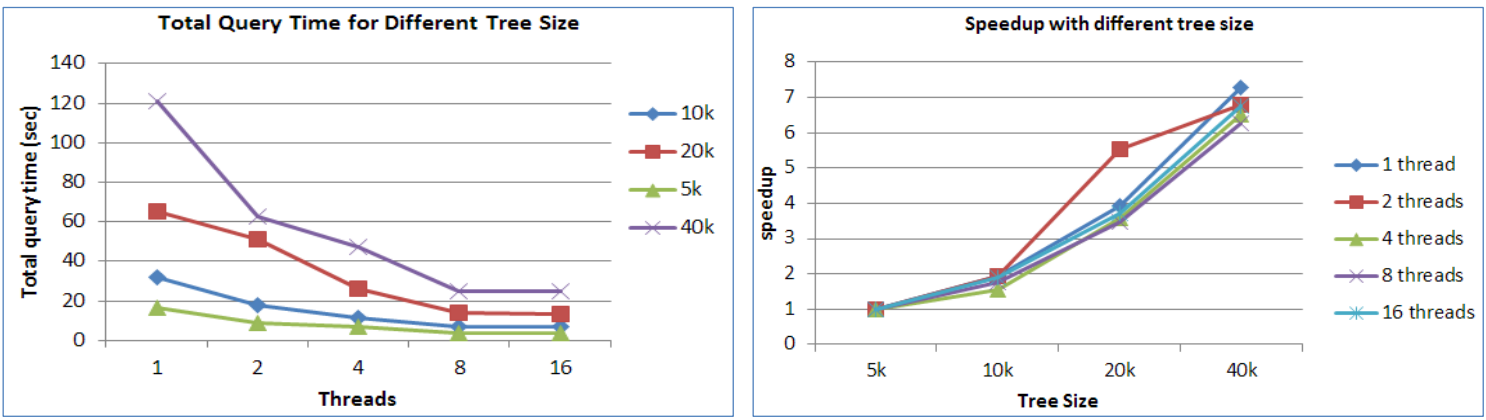

Figure 5.11: increasing the size of initial tree

40000 (40k) data rows. For each tree, we executed 1000 queries mixed with 200 insertions. The experiment results in figure 5.11 illustrate that the total query time is increased by about $50 \%$ when the size of the initial tree is increased by $50 \%$ from $5 \mathrm{k}$ to $10 \mathrm{k}$ and from $10 \mathrm{k}$ to $20 \mathrm{k}$. However, when the tree size was increased by $50 \%$ from $20 \mathrm{k}$ to $40 \mathrm{k}$, the total query time is increased by about $45 \%$. Therefore when the initial tree size is increased, the response time for each query can be reduced. In the PDC program, the initial tree building was implemented with a single thread, therefore, with the selected instances, it took more than 6 hours to build an initial tree containing over 40k data. We didn't continue the tests with bigger sample data.

- Test4: Initial tree size 10,000 rows, 1000 queries, 0 insertion/200 insertions, and $25 \%$ output coverage for different number of children nodes capacities on different machines 


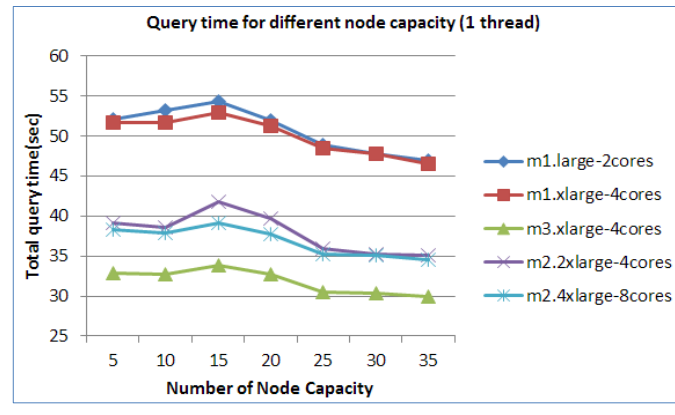

Figure 5.12: 1 thread/0 insert/1000 queries

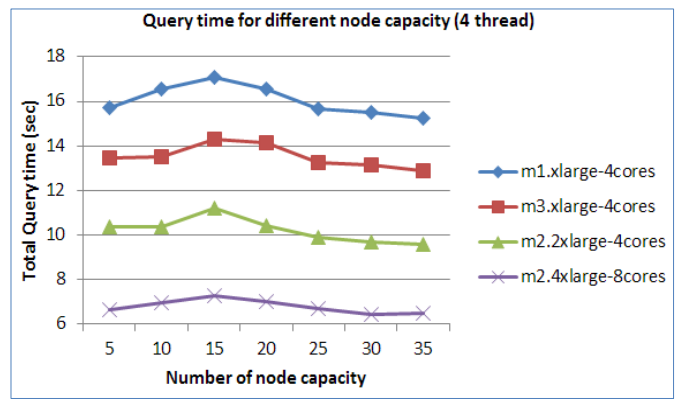

Figure 5.14: 4 threads $/ 0$ insert/1000 queries

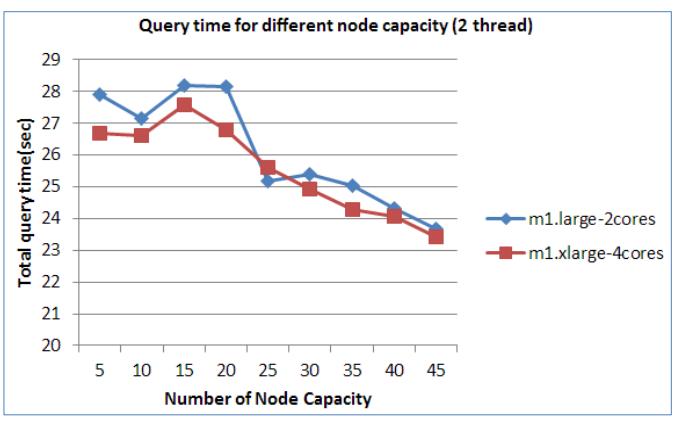

Figure 5.13: 2 threads/0 insert/1000 queries

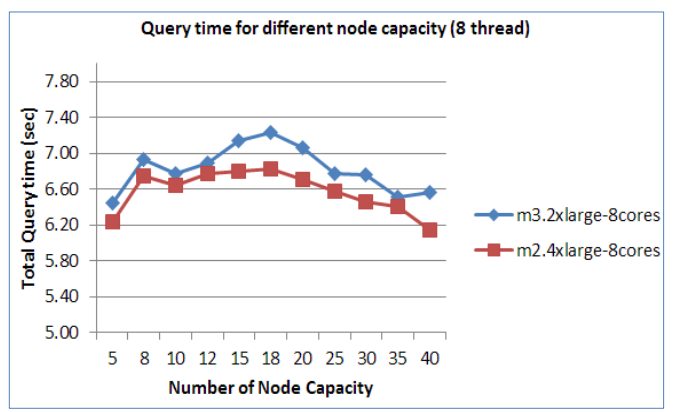

Figure 5.15: 8 threads /200 insert/1000 queries

In this test, we evaluated the system performance when the directory node capacity increases from 5 to 35 . This experiment demonstrates that the directory node capacity has an impact on the query time. We notice that the query time reaches the maximal value when the capacity is 15.(See figure 5.12, 5.13, 5.14 and 5.15) When node capacity is less than 15 , the more children nodes a directory node has, the more time query execution needs. When node capacity is larger than 15 , the more children nodes a directory node has, the less time query execution needs.

This result was brought to our attentions. In order to further evaluate how the node capacity influences the performance, I performed the following tests on the VCL cloud.

- Test5: Initial tree size 100,000 and 50,000 rows data, 1000 queries, 0 insertions with $25 \%$ output coverage on the HPC CentOS machine by increasing the node capacity 
from 5 up to 1920.

In this test, we evaluated the number of node capacity which can result in the best system performance when the data size was reduced by half. In figure 5.16, the maximal total query time is 665 seconds when the node capacity reaches 90 for a tree in size of 100,000 rows. In figure 5.17, the maximal total query time is $356 \mathrm{sec}-$ onds when node capacity reaches 60 for a tree in size of 50,000 rows. The minimal building time for the tree in size of 100k rows and 50k rows are 197 seconds and 78 seconds respectively. Therefore, the insertion time reaches optimal when the directory node has 60 children nodes. However, the query time may be the worst at the capacity of 60 . We also noticed that when the sample data was reduced by half, the node capacity was decreased but not by the same ratio (50\%) to achieve the best performance.

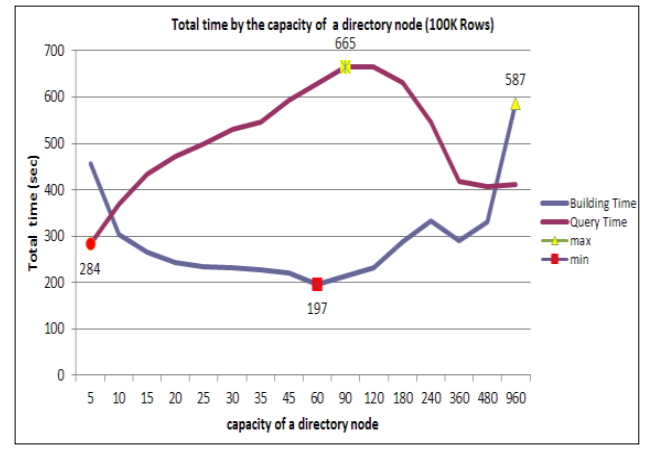

Figure 5.16: 100k data Insertion and 1000 query time with $25 \%$ coverage $(5<=$ capacity $<=960)$

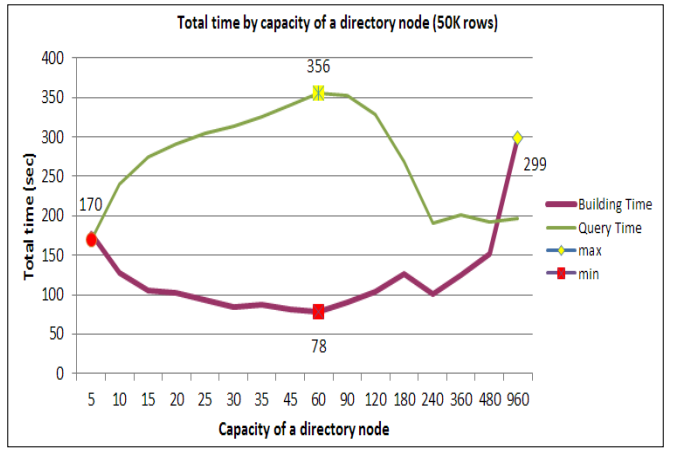

Figure 5.17: 50k data insertion and 1000 query time with $25 \%$ coverage $(5<=$ capacity $<=960$ )

The reason for having such a behavior could be: the overlap between the nodes become small when the node capacity is larger than a certain point. When the capacity is very large, the tree is very flat. The number of MDS ids in the MDS set of each directory node becomes small so a query MDS has a higher possibility to contain a directory node's MDSs in the higher part of the tree. The measure of this directory node can be added to the result and search does not go down very deep in the tree. Thus, less time is used to return the query results. But when handling insertion, after certain point the new data's MDS has a smaller possibility to overlap with a directory 


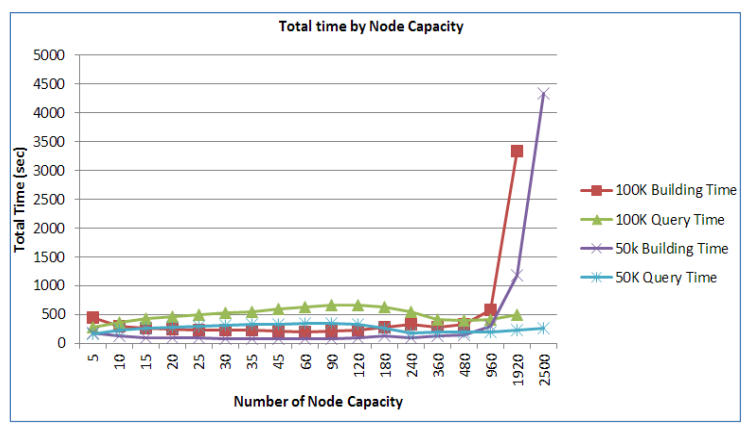

Figure 5.18: $100 \mathrm{k}$ data Insertion and 1000 Query with $25 \%$ coverage $(5<=$ Capacity $<=$ 2500)

node's MDSs. The new data's MDS needs to be compared with more directory nodes and more MDSs in a directory node. This is time consuming and it slows down the operation. Hence, it takes more time to find a subtree to trace down and insert a new data node. Obviously, there is a trade-off on selecting the node capacity in order to optimize the total execution time cost.

In test 4 , we observe the query time is the worst when the node capacity is 15 for a tree size at $10 \mathrm{k}$ rows. In test5, we get the worst query time when the node capacity is 60 for a tree size in 50k rows and 90 for a tree size in 100k rows. This shows that the directory node capacity can vary for different scale data size in order to achieve optimal speedup.

In addition, in figure 5.18 we also observe there is a big jump in building time when the node capacity exceeds 960. It is taking more than an hour to build a tree with single thread while a directory node contains 2500 children nodes. As it said, the MDS in directory nodes on each tree level has very small overlap or no overlap with each other when the capacity is very large. The insertion process has to scan most directory nodes on each level one by one in order to find a suitable subtree to trace down for further comparison.

Figure 5.19 shows the number of node splits are happened and the number of directory nodes are created as well as the number of MDS expansions are occurred during the initial tree building. When the node capacity is increased, the number of 

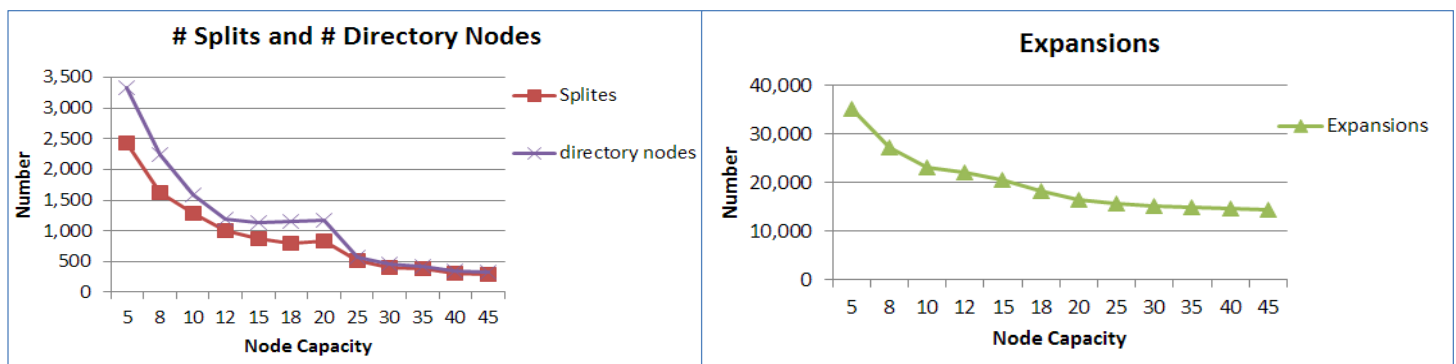

Figure 5.19: Number of node splits,directory nodes, MDS expansion,

directory node and the number of splits are decreased, so does the number of MDS expansions.

Similar experiments are also performed on larger data set containing 1 million data tuples with 1000 queries for 5\% coverage and 25\% coverage. In order to observe the impact resulted by directory node capacity only, we used a single thread for the experiments. Figure 5.20 and 5.21 demonstrate that the query total time gets increased when the node capacity is increased, and the insertion time fluctuated while changing the number of capacity. However, when the capacity is around 60 the system reaches optimal performance for insertions, and when the capacity is around 10 the system achieves the best performance for query operations with both $5 \%$ and $25 \%$ coverage.

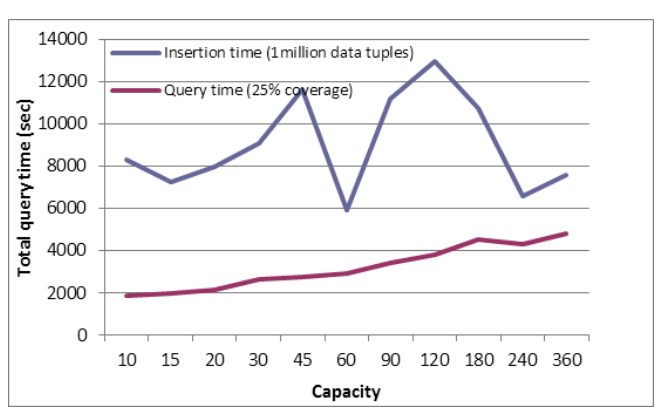

Figure 5.20: 1 million data insertion and 1000 queries with $25 \%$ coverage $(10<=$ capacity $<=360) \%$ coverage $(5<=$ capacity $<=960$ )

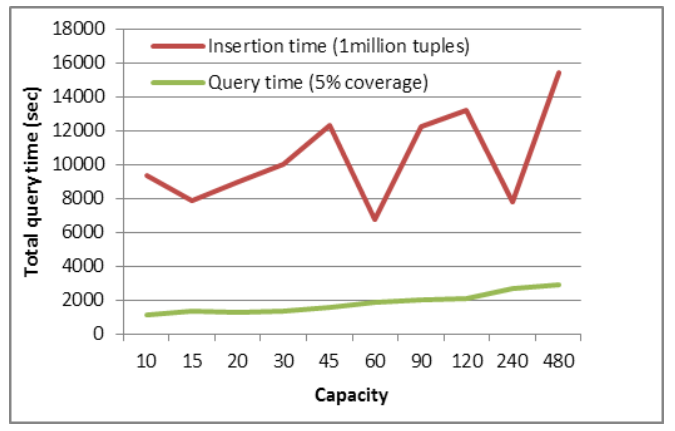

Figure 5.21: 1 million data insertion and 1000 queries with $5 \%$ coverage $(10<=$ capacity $<=480$ ) 


\subsection{Summary}

The DC-tree provides sequential solution to high dimension OLAP data. The PDC-tree parallelizes the queries and insertions on multi-core processor by modifying the DC-tree so it is able to processing large data sets. The experiments show that PDC-tree can handle 130 transactions every second with 16 cores but only 12 transactions with single core. The amount of data processed with 16 cores is 10 times more than the amount processed with 1 core. The process of each transaction with 16 threads is 0.0074 seconds on a 10000 -row data set, which is 10 times faster than it is with single core. All the tests demonstrate that the system has increasing speedup when the number of cores is increased. In conclusion, the PDC-tree index structure provides the potential solution to build a real-time OLAP.

In addition, in the implementation of PDC-tree the MDS is represented in strings and stored in a set container. Range queries, insertions, and nodes splits all require MDS comparison. The system spends lots of time on MDS string comparison. If MDS can be represented in a more efficient data type, we expect to obtain better results. To improve the performance, in the design of Parallel real-time OLAP on cloud based distributed system, we use bit representation and unorder set container to store MDS. The details will be introduced in the next chapter. 


\section{Chapter 6}

\section{Real-time OLAP on cloud based distributed platform}

\subsection{Introduction}

Cloud is typically a platform that allows services to scale on-demand and perform failure recovery. Elasticity is one of many advantages of the cloud. It enables users to acquire or release computing resources dynamically depending on demand. Moreover, a cloud can efficiently support large volumes of data. Parallel computing is used to develop applications for cloud environments.

But BI and the OLAP systems usually are highly resource intensive [16]. In self-hosted environments, the never ending expanding requirements of an OLAP system often are a critical issue for many companies. Cloud infrastructures provide the alternative platforms for BI applications.

A real-time OLAP on a distributed cloud platform requires a good strategy to partition big data into small sets. Multiple processors can process those small data sets concurrently. The system should catch up any data update and add it to OLAP databases in real-time for users to perform query requests. The nearly new data should be included in search results even if it is newly added just right before a query request being sent. Such a real-time OLAP system can be accessed by a large number of users at the same time. It requires a capability to process transactions such as data insertions and user queries in parallel. The system should be efficient enough to response any user's requests in a short time.

In Chapter 5, we introduced PDC-trees that handle OLAP transactions on multi-core processors concurrently. To design a parallel real-time OLAP on a distributed cloud platform we extended the PDC-tree data structure so that the whole tree can be divided to several subtrees and the subtrees can be distributed to different cloud nodes. Any user queries and new data insertions are processed in parallel not only within each node but also in multiple nodes. By replacing MDS set used in PDC-tree with MDS Range, the tree performance is improved. This is why the distributed tree structure is called PDCR-tree. 
The parallel real-time OLAP is built with $m+1$ machines in a cloud environment. Each machine may have multiple cores to run up to $\mathrm{k}$ threads. We call this system CR-OLAP (Cloud based Real-time OLAP system). In the following, we present the detailed design of the CR-OLAP and its algorithms to handle parallel processing.

\subsection{Overview of PDCR-tree design}

The CR-OLAP runs on multiple nodes provided by cloud service providers. It contains several components including a distributed PDCR tree on $\mathrm{m}+1$ nodes, a network communicator, a load balancer, a migration API and a message serialization API.

The building of PDCR-tree starts on one node called master. When the tree grows big enough, we start building the subtrees on other nodes called workers. After the initial load, the whole PDCR-tree is distributed on multiple nodes. The master contains the top part of the tree and we call it hat. Each worker contains a number of subtrees and each subtree is the same as a small PDCR-tree. After the initial built, the system is ready to take client requests such as new data insertions and range queries. The master node also maintains the information of distributed subtrees. It uses a lookup map to record which workers the subtrees are stored. When the master receives requests, the requests will be executed in parallel in the hat. If the master need to dispatch the tasks to workers, it will look up its subtree-worker map to find the workers storing the relevant subtrees. Each worker who receives its tasks will complete the tasks in parallel locally and return the results back to the master. When the master node gathers all results returned by the workers, it generates a final result for the request. The final result are sent over to the client at the end.

A load balancer runs periodically and moves subtrees from workers with heavy loads to workers with lighter loads. All instructions to trigger tasks are represented as messages (for example,migration request, sending subtree, query request and insertion request). Each message carries its own data members such as a destination worker id, a source worker id and task instructions. Sending or receiving messages between nodes is handled by a network communicator. We implemented a communicator with ZeroMQ, a high-performance asynchronous messaging library. Every message is serialized to a string by a message serializer and pushed to a message queue. Then, the network communicator is responsible for retrieving messages from the queue and sending them to their destination nodes. Once a 


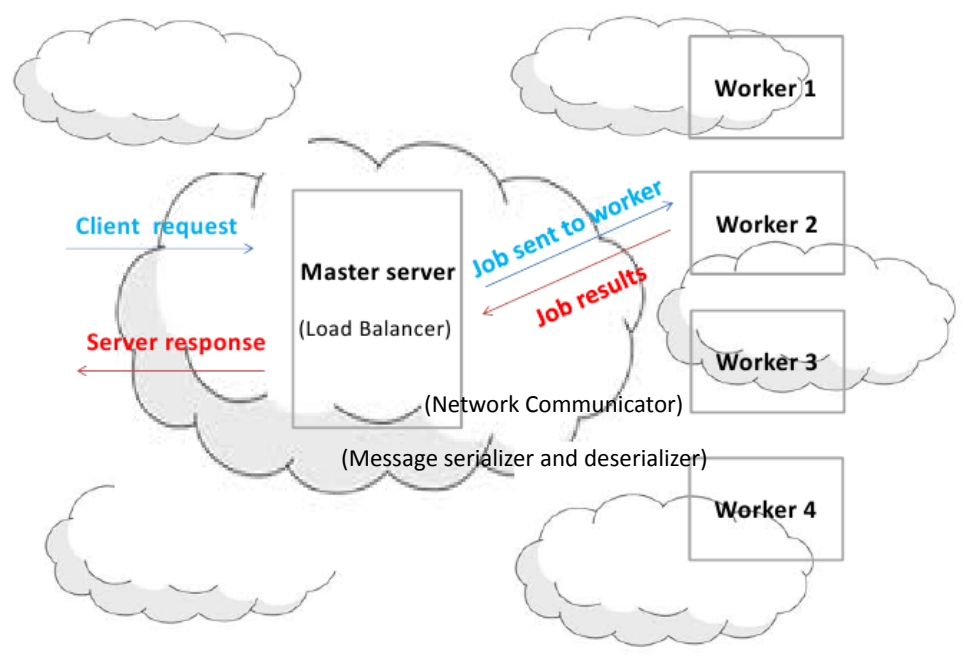

Figure 6.1: System architecture

destination node receives a message the message de-serializer de-serializes the string and recovers the original message. Then the worker performs the message task. Figure 6.1 demonstrates the architecture of the system.

\subsubsection{Distributed PDCR tree and its data structure}

The PDCR-tree is stored on cloud nodes. Each cloud node stores several subtrees of data. A distributed PDCR tree is a data structure containing a hat and a set of subtrees $T, T=\left\{t_{1}\right.$, $\left.t_{2}, t_{3} \ldots t_{i}\right\}$, where $t_{i}$ is a subtree. The hat is stored in the master node and the subtrees are distributed between several worker nodes. Figure 6.2 demonstrates a distributed PDCR-tree with a hat in the master and subtrees in several workers.

\section{HAT:}

The hat is the top part of the distributed PDCR-tree containing the root of the whole tree and first a few levels of the tree. The depth of a hat is defined by a parameter CUT. We can define different value for CUT. The hat contains directory nodes and data nodes. The non-leaf nodes in the hat are directory nodes and the leaf nodes in the hat can be either directory nodes or data nodes. When the depth of a directory node is smaller than a CUT level, the leaf-node is data node; When the depth exceeds a CUT level, the data node is moved to workers. In this case, the leaf node becomes a directory node. When the 


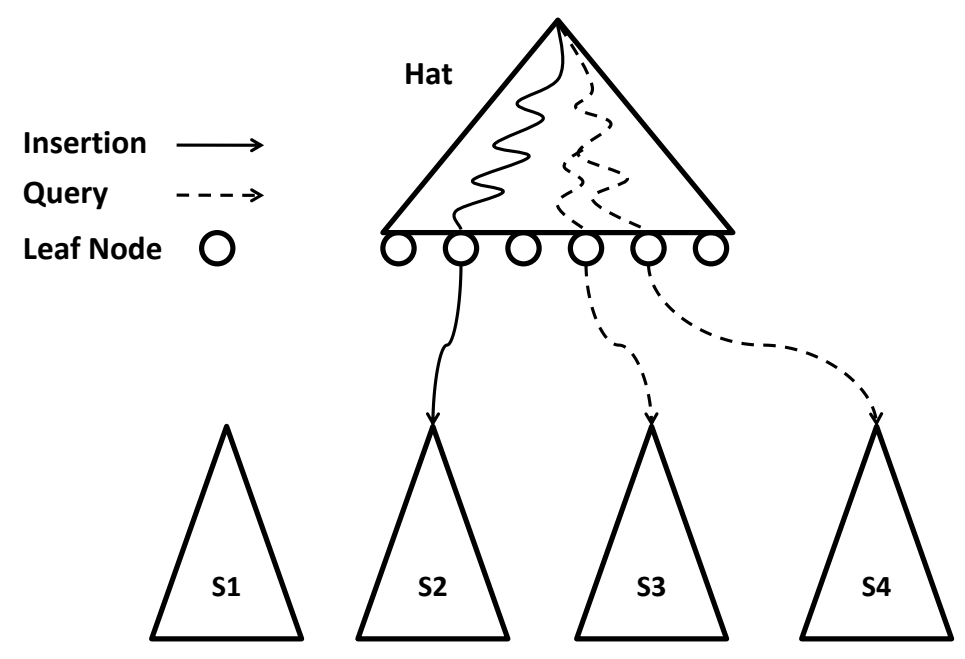

Figure 6.2: Distributed PDCR tree in master and workers

distributed PDCR-tree is small enough to be stored only in the hat, it is a regular PDCRtree. When the tree grows big enough to have subtrees distributed in worker nodes, the hat only contains directory nodes and the leaf nodes are the parent of subtrees stored in workers.

\section{Subtrees:}

The subtrees are part of a distributed PDCR-tree under a CUT level. They are stored in worker nodes and are the children of the leaf nodes in a hat. Each subtree contains a root node, directory nodes and data nodes. The root of a subtree is also a directory node in the whole distributed PDCR-tree. A subtree is also a PDCR-tree. However, the root node of a subtree may have the link to the root of its sibling subtree. A directory node of a subtree has the same structure as a directory node of a hat. A leaf node of a subtree is a data node. Figure 6.3 shows the data structure of a directory node. Figure 6.4 shows the data structure of a data node. The data structure of a data node only contains the MDS and Measures. The data structure of a directory node contains the MDS Range, the Timestamp, Measures, Links to backup, Links to parents, Links to siblings and Links to children.

The data nodes and directory nodes are stored in two separate arrays. Any new element is added to the end of arrays. Each directory node or each data node in their arrays has an index. Members such as "Links to backup", "Links to parents", "Links to siblings" and "Links to children" in the directory node data structure stores the indices of the node 


\begin{tabular}{|l|l|c|c|c|c|c|c|}
\hline MDSR & Creation & Update & Measure 1 & Link to & Link to & Link to & Link to Child 1 \\
/MDS & TimeStamp & TimeStamp & $\ldots$ & backup & parent & sibling & $\ldots$ \\
Link to Child n
\end{tabular}

Figure 6.3: Data structure of directory node

\begin{tabular}{|l|l|l|l|}
\hline & & & \\
MDS & Creation & Update & Measure 1 \\
& TimeStamp & TimeStamp & $\ldots$ \\
& & & Measure n \\
\hline
\end{tabular}

Figure 6.4: Data structure of data node
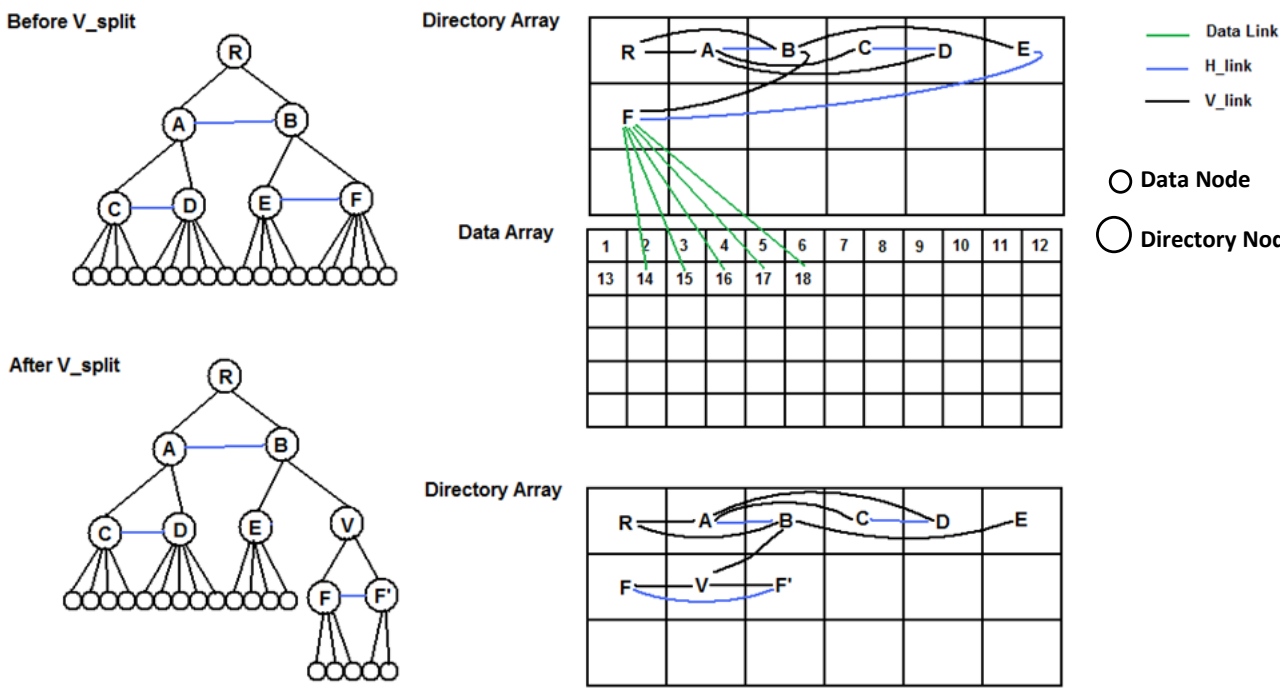

O Data Node

Directory Node

Directory Array

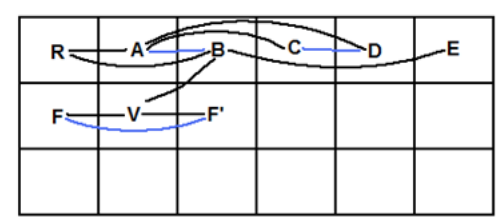

Figure 6.5: Array structure of data nodes and directory nodes 
elements. The array based data structure can easily be compressed and transferred between cloud nodes via message passing.

The figure 6.5 illustrates the directory node array and the data node array before and after a node split. A directory node ' $R$ ' stores indices of the directory nodes ' $A$ ' and ' $B$ ' as "Links to children". the directory node 'A' stores the index of the node 'B' as "Links to siblings". The directory node 'F' stores indices of the data nodes ' 14 '- '18' as "Links to children". Before the node ' $F$ ' is split the directory node ' $B$ ' stores indices of ' $E$ ' and ' $F$ ' as "Links to children"; after the node ' $F$ ' is split, the "Links to children" in the directory node ' $\mathrm{B}$ ' is updated. As a result, the index of ' $\mathrm{F}$ ' is deleted and the index of ' $\mathrm{V}$ ' is added.

\section{MDS and MDSR:}

In a directory node, an MDSR is a range of a MDS set. As we introduced in Chapter 5 , in the PDC-tree, a directory node stores a set of MDS. Each MDS has an ID that represents the data attributes in a hierarchy schema of a dimension. For example, assume we have a data node $X\{0001,20130501, \mathrm{c} 001, \$ 5000\}$. It has 3 dimensions (store, sales_date, product) and 1 measure (sale_amount). The fact table stores a surrogate key from each dimension. The hierarchy schema for the dimension "store" is County-ProvinceCity-StoreId and the MDS for '0001' can be represented as Canada-Ontario-Ottawa-0001; The hierarchy schema for "date" dimension is Year-Month-day-dayId and the MDS for '20130501' can be represented as 2013-05-01-20130501; The hierarchy schema for "product" dimension is Category-Brand-Class-ProductId and the MDS for 'c001' can be represented as Electronics-Samsung-SmartPhone-c001. If a directory node D only contains this data node X, D's MDS set is \{Canada-Ontario-Ottawa-0001, 2013-05-01-20130501, Electronics-Samsung-SmartPhone-c001\}. When we have another data node Y $\{0002$, 20130523, a001, \$6000\} as one child of this directory node D, the MDS set of the directory node $\mathrm{D}$ is $\{\{$ Canada-Ontario-Ottawa-0001,Canada-Ontario-London-0002 $\},\{2013-05-01$ 20130501,2013-05-23-20130523\}, \{Electronics-Samsung-SmartPhone-c001, Electronics-AppleSmartPhone-a001\}\}

However, when the data size is growing, the MDS set size becomes large in each directory node. Every time, when an insertion or a range search request comes, the MDS Ids of a new data or a range query needs to be compared to all Ids in the MDS set in each directory node in order to perform the insertion or answer the query. In addition, when an insertion 
necessitates a node split a comparison of MDS Ids between all children directory nodes is required in order to redistribute the children nodes and achieve minimal MDS overlap between the split nodes. Such string comparison is very time consuming. To reduce the time on comparing MDS Ids, we can adapt the MDS to the higher level to reduce the number MDS ids in directory nodes. For example, if we have 20 data tuples which are the sales of all stores in Ontario and are all about Samsung products in 2013, we can adapt all lowest level MDS Ids to higher level MDS Ids \{Canada-Ontario, 2013, Electronics-Samsung\}. The MDS set size is reduced to 3 . Nevertheless when the cardinality of the distinct values at each hierarchy level is large, there can still be many MDS Ids in the directory nodes. Such MDS representation can make the system slow.

\begin{tabular}{|c|c|c|c|c|c|}
\hline & \multicolumn{2}{|c|}{$\longleftarrow \mathrm{n}_{0}$ bits $\rightarrow \longleftarrow \mathrm{n}_{1}$ bits } & & $\longleftarrow n_{i}$ bits & $\rightarrow \leftarrow n_{i+1}$ bits $\longrightarrow$ \\
\hline & Level 0 & Level 1 & ... & Level $\mathrm{i}$ & Level $\mathrm{i}+1$ \\
\hline MDS & Store Dim & Canada & Ontario & Ottawa & 0001 \\
\hline MDS ID Store\#1 & 0001 & 0000010 & 00000101 & 00000100 & 1001001 \\
\hline MDS ID Ottawa & 0001 & 0000010 & 00000101 & 00000100 & 0000000 \\
\hline MDS ID Ontario & 0001 & 0000010 & 00000101 & 00000000 & 0000000 \\
\hline MDS ID Canada & 0001 & 0000010 & 00000000 & 00000000 & 0000000 \\
\hline
\end{tabular}

Figure 6.6: MDS IDs in bit representation

Therefore, we modified the MDS in the PDCR-tree to improve performance. One improvement we made is the MDS Id representation. Instead of using a string to represent the MDS Id in the PDC-tree we use bits in the PDCR-tree. We choose $\mathrm{n}_{i}$ bits for each hierarchy level $\mathrm{i}$ and $\mathrm{n}_{i}$ can be any number. The number of bits depends on the the data being represented in the fact table. We assign a value to a $\mathrm{n}_{i}$-bit string for a value at hierarchy level $i$. The MDS Id at level $i$ is set to the concatenation of the MDS sub-id of level $i$, the MDS sub-ids at level $i-1$ or higher and the MDS sub-ids at level $i+1$ or lower which contain only 0s. Figure 6.6 demonstrated the id construction for an MDS. To set an MDS Id for 
"Ontario" at the Province level, we concatenate the sub-ids at the Country and Province levels along with the City and StoreId MDS sub-ids that only contain 0s.

The StoreId 0001's MDS Id can be adapted to its higher level MDS Id Ontario by replacing $0 \mathrm{~s}$ in the levels lower than Province. Because the $\mathrm{n}_{i}$ bits value can also be read as integers, instead of comparing strings of MDS Ids in the PDC-tree, we now compare two integers of MDS Ids in the PDCR-tree. Comparing integers is faster than comparing strings so it will improve the PDCR-tree performance.

The other improvement we made is replacing a set of MDS ids in a directory node with a range of MDS (MDSR) ids for dimensions. In the DC-tree and the PDC-tree, partial ordering is used for representing data regardless it is ordered or unordered because the hierarchies define the relationship between levels (the value in a higher level contains the value in a lower level), therefore, the unordered data can be grouped by their relevant hierarchies. In the R-tree, the MBR is used to describe spaces. Usually, ordered number values (e.g. $1,2,3 \ldots$ ) are used to represent a space size since space dimensions are all ordered. We took the idea of MBR and applied lower bound and upper bound to define the range of MDS set. The lower bound and upper bound are represented by MDS Ids.

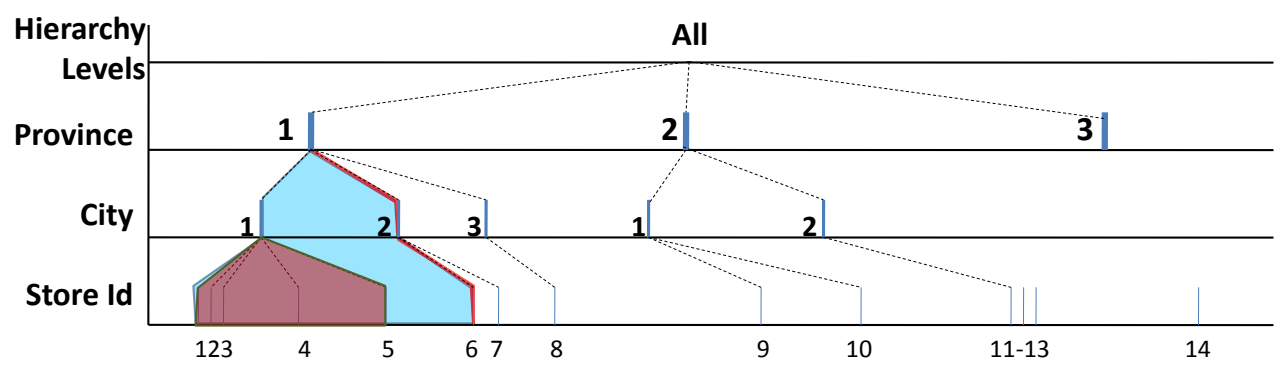

Figure 6.7: An example of relationship between hierarchy levels of a given dimension

Instead of listing every MDS of each dimension in the set, we assign the range of MDS to describe the directory node. This allows representing unordered dimension in an ordered manner. Figure 6.7 demonstrates the hierarchical relationship of the unordered dimension "Store". As illustrated, province ' 1 ' has cities ' 1 ' to ' 3 ', and city ' 1 ' includes ' 1 ' to ' 5 ' stores. The Ids ' 110 ' to ' 130 ' are the boundary of MDS set at the City level. The Ids ' 111 ' to ' 115 ' are the boundary of Store belonging to City ' 110 '. If a range query is searching all stores in city ' 110 ', we can compare MDS '110' with the MDSR of city (for example 
['110', '130']) to find the right directory node to be processed.

We use such an MDS Range (MDSR) in the directory node of the PDCR-tree. When traversing the directory nodes, we compare MDS Ids of a query or of a new data with the upper bound and the lower bound of MDSR in the directory nodes. Therefore, at each directory node, only two comparisons are performed for each dimension. The performance is better in the PDCR-tree than it is in the PDC-tree. This is similar to the R-tree that uses an MBR to define a sub space. However, an MBR is not able to represent unordered dimensions.

\subsubsection{Building distributed PDCR tree from a master to workers}

Starting from the root directory node on the master, PDCR-tree is growing by inserting new data one by one. A directory node has its node capacity, for example, maximum 15 children nodes. When the number of children nodes exceeds the node capacity, a node split will be performed. There is two types of node split: vertical split (V-Split) and horizontal split (H-Split).

An H-Split is performed when the number of children nodes of a directory node is full, but the parent node of this directory node can still contain more children nodes. A horizontal link will be added between the two split nodes. For example, in figure 6.8 (a), the directory node capacity is 2 and the Cut level is $1 . \mathrm{R}$ is the root. A is the directory node with depth 1 and contains 2 children nodes. If a new data were inserted to the node A, A would contain 3 nodes which exceeds its children capacity, but A's parent node $\mathrm{R}$ has not reach its node capacity yet, therefore, An H-split can be performed and split A to two nodes A and B ( figure 6.8(b)). A has the sibling node link pointing to B. The 3 children nodes are distributed between A and B that leads to minimal MDS overlap between A and B. 


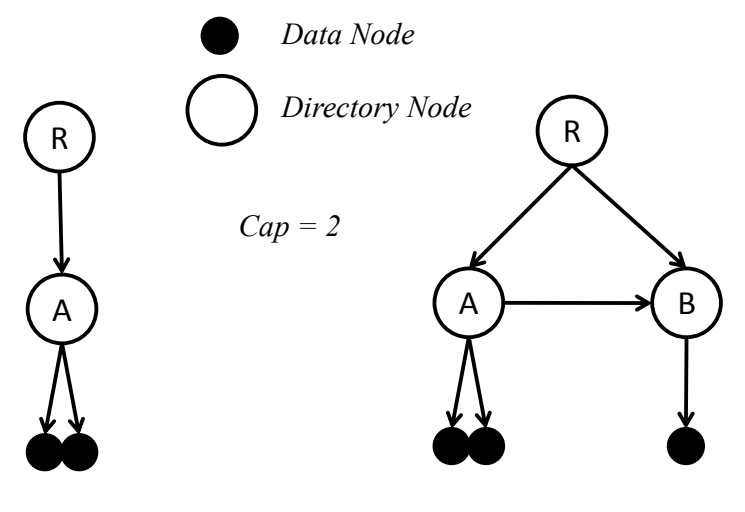

(a)

(b)

Figure 6.8: Vertical split during a new data insertion

If a directory node reaches its node capacity and its parent node also contains children with full capacity, both a V-Split and an H-Split will be triggered. An H-split along with a V-split can make the two subtrees become independent. The directory node gets pushed 1 level down in the tree. In figure 6.9(a), if a new data were added into node A, the number of children nodes of A would exceed the capacity and the number of children nodes of R, the parent of A, also would exceed the capacity; hence, both the H-split and the V-split are called. The H-Split splits A to A and $\mathrm{A}^{\prime}$; The V-Split adds a new node $\mathrm{C}$ as the parent of $\mathrm{A}$ and $\mathrm{A}^{\prime}$ and links $\mathrm{C}$ to the parent node $\mathrm{R}$ which $\mathrm{A}$ linked to before the split. The link between $\mathrm{A}$ and $\mathrm{B}$ from previous $\mathrm{H}-\mathrm{Split}$ can be removed. The subtrees $\mathrm{C}$ and $\mathrm{B}$ become independent. $\mathrm{A}$ and $\mathrm{A}^{\prime}$ is at the depth level of 2 of the hat on the master.

Once the depth of directory node exceeds the Cut level, the directory node with its children nodes will be moved to the worker node. In figure 6.9(c), the depth of $\mathrm{A}$ and $\mathrm{A}^{\prime}$ exceeds the Cut level, so $\mathrm{A}$ and $\mathrm{A}^{\prime}$ with their data nodes are moved to a worker node. Figure 6.9(d) and (e) are showing the continuously growing of the PDCR-tree. A new data is added to $\mathrm{A}$ on a worker and both the $\mathrm{V}$-split and the $\mathrm{H}$-split is executed. A gets split to $\mathrm{A}$ and $\mathrm{A}^{\prime \prime}, \mathrm{D}$ is the result of the $\mathrm{V}$-split. The link from $\mathrm{A}$ to $\mathrm{A}^{\prime}$ is removed and the subtree $\mathrm{D}$ and $\mathrm{A}^{\prime}$ is now independent. We also move one of the subtree (e.g. the subtree of $\mathrm{A}^{\prime}$ ) to another worker so the work load is spread out between workers. This is a process of migration. More details will be introduced later. 


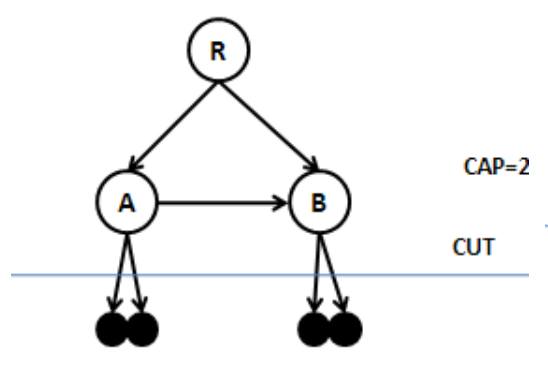

(a)

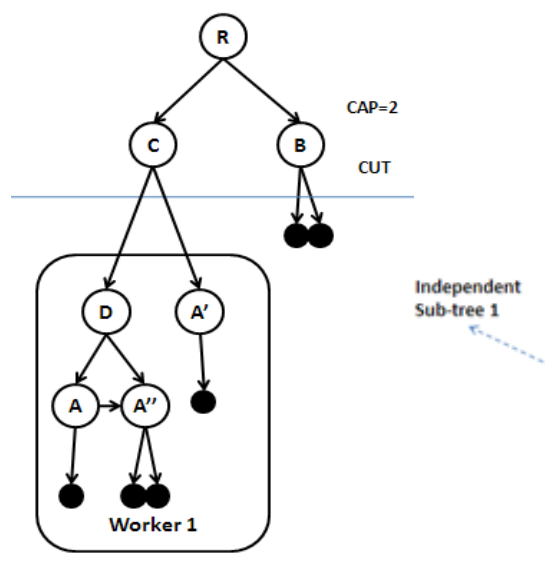

(d)

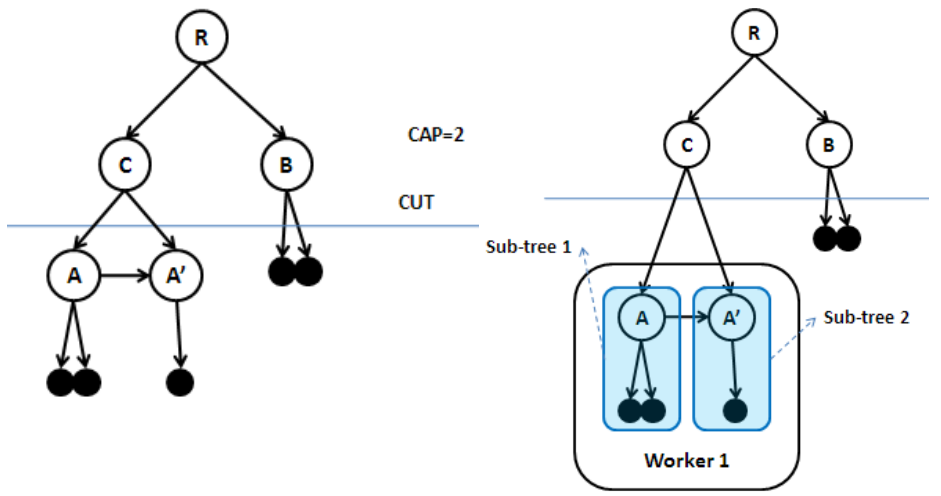

(b)

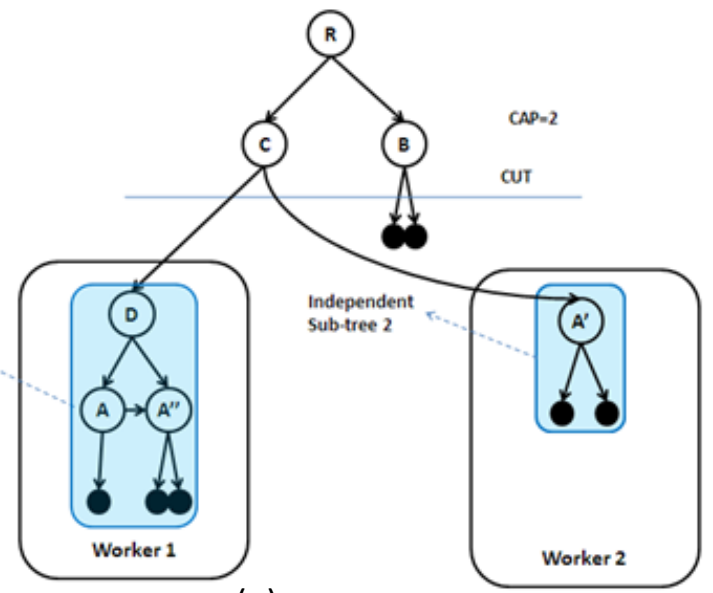

(e)

(c)

Figure 6.9: PDCR-tree growing from master to a worker: (a) Tree with four data nodes; (b) Vertical node split performed when node A exceeds capacity; (c) the subtree is moved to worker when the depth of tree exceeds cut level; (d) a vertical split performed on node A in worker; (e) subtree 1 and subtree 2 becomes two independent subtrees after the vertical split and subtree $\mathrm{A}^{\prime}$ is moved to worker 2

\section{The Proof of Independent subtree:}

When a V-split happens, the subtree of the new node generated from split becomes independent from the neighbor nodes which the original node linked to or be linked from before the action is taken. The proof of this statement is followed.

Assume a subtree rooted at node $\mathrm{N}$ is call $T_{N}$, where $\mathrm{N}$ is a directory node in the PDCR tree. In figure 6.10(a), $\mathrm{T}$ is a subtree containing root $\mathrm{t}$ and $\mathrm{P}, \mathrm{A}, \mathrm{B}, \mathrm{C}$ is directory nodes in $\mathrm{T}, T_{P}$ is a subtree in $\mathrm{T}$, and $T_{A}, T_{B}, T_{C}$ is also subtrees in $T_{P}$

Lemma 1: Assume a query search is traversing $\mathrm{T}$ and $T_{B}$, if any updates or splits happen to node $\mathrm{B}$, the sibling nodes of $\mathrm{B}$ can be found through horizontal sibling links and 


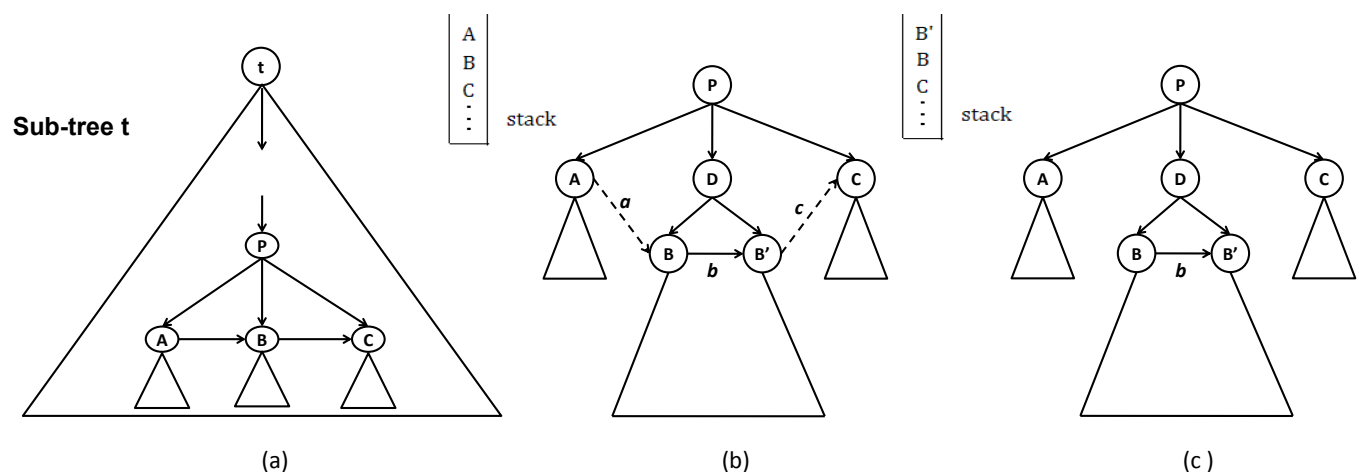

(a)

(b)

(c)

Figure 6.10: a subtree becomes independent after vertical node split: (a) Subtree B root with sibling links; (b) Node B has vertical split and creates Node D; (c) Subtree D resulted from vertical split does not have links with neighbor nodes

be pushed into a stack for re-processing the subtrees.

\section{Theorem 1:}

Assumption: Assume $\mathrm{P}$ is the parent directory node of A, B, C in t (Figure 6.10(a)) and $\mathrm{A}, \mathrm{C}$ are the left and right sibling nodes of $\mathrm{B}$. A vertical split, as the result of a horizontal split in node $\mathrm{B}$, creates a node $\mathrm{D}$ which replaces node $\mathrm{B}$ and is set to be the parent of node $\mathrm{B}$ and its new sibling node $\mathrm{B}^{\prime}$. (Figure 6.10(b))

Proposition: Any query q traversing the sub-tree $\mathrm{t}$ does not need the horizontal links a and $\mathrm{c}$ to detect the updates in $T_{D}$.

Proof: Define B as the modified node B after updates in figure 6.10(b), and $\mathrm{B}_{\text {stack }}$ as the old node B pushed into stack before updates.

Case 1: Query q does not have B or one of its ancestors in its stack.

a. The query does not need visit $\mathrm{B}$. Therefore, $\mathrm{q}$ does not need to process any updates in $\mathrm{B}$.

b. The query has not yet visited node B to push it into its stack. In this case, the query will first visit the newly created node $\mathrm{D}$ and pushes it into the stack. This will cover all children of $\mathrm{D}$ including $\mathrm{B}$ and $\mathrm{B}^{\prime}$ while evaluating node $\mathrm{D}$.

Case 2: Query q has B or one of its ancestors in its stack. 
a. If q pops node A from the stack, it has not yet popped B from its stack. B is still in the stack waiting for processing. Any updates happen to B in the subtree will be detected when B is popped up from the stack for processing. Therefore, the horizontal links a and c are not used for re-processing.

b. If q pops node B from the stack, it compares the time stamp of $\mathrm{B}_{\text {stack }}$ and $\mathrm{B}$. $q$ detects the difference between the time stamps, hence, using the horizontal link $b$, the new sibling node $\mathrm{B}^{\prime}$ will be found and pushed into the stack with $\mathrm{B}$ together for re-processing. Note that it is not possible to pop node $\mathrm{B}^{\prime}$ since it did not exist in the tree when q visited node B before the updates, so it could not be pushed into the stack. Node $\mathrm{C}$ is still in the stack and will be popped later. Therefore, the horizontal links a and c are not used for re-processing.

c. If q pops node $\mathrm{C}$, it ignores any update in $\mathrm{B}$, since these updates occurred after processing of $\mathrm{T}_{B}$ was complete. Horizontal links a and $\mathrm{c}$ are not used for reprocessing.

d. If an ancestor of B is in the stack, B will be pushed later into the stack and the above cases a-c will be applied to it.

Therefore, it is proved that a query q can detect any updates of $\mathrm{T}_{B}$ and push the old and the new nodes resulted by V-Splits for re-processing. Horizontal links a and $\mathrm{c}$ are not not necessary for the re-processing.

According to Theorem 1, the horizontal link (i.e. link a) from one subtree (e.g. $T_{A}$ ) to the old root of a subtree (i.e. $T_{B}$ ) can be safely removed during a vertical node split, because it is not used for re-processing of the updated nodes. For the same reason, the horizontal link (i.e. link c) from the new sibling node $\left(\mathrm{B}^{\prime}\right)$ to other subtree (i.e. $T_{C}$ ) can be safely removed. Figure 6.10(c) shows the subtree structure after a V-split.

Lemma 2: It is not necessary to create the new horizontal links from node $A$ to $D$ and node $\mathrm{D}$ to $\mathrm{C}$ during $\mathrm{V}$-Splits in order to cover updates that occurred in the meantime.

Proof: In all cases mentioned in Theorem 1, queries are able to detect updates with the horizontal link $b$, and using the time stamps in directory nodes. Hence, there is no need to create horizontal links between nodes A, D and C.

Theorem 2: 


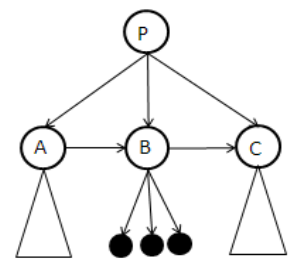

(a)

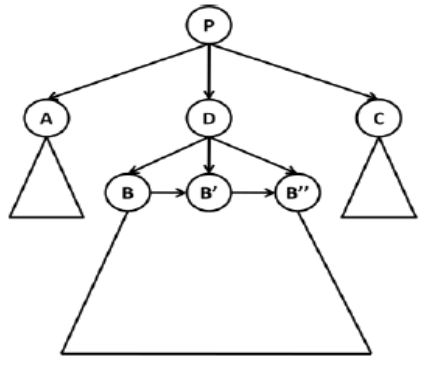

(d)

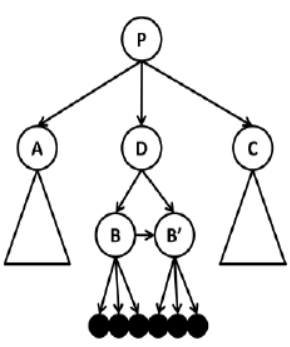

(b)

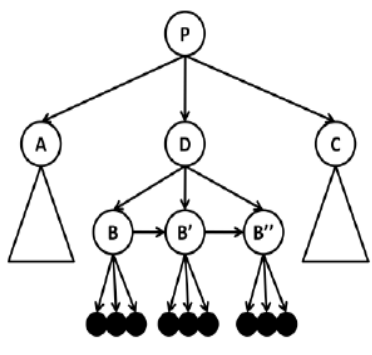

(c)

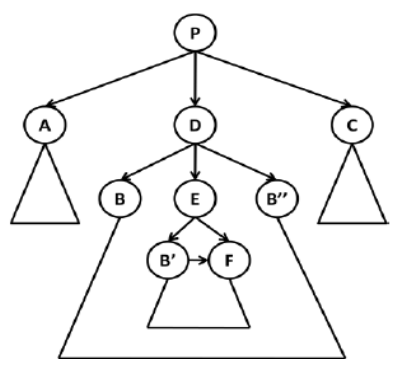

(e)

Figure 6.11: Subtree becomes independent to any neighbor nodes

Assumption: Assume in sub-tree $\mathrm{T}, \mathrm{P}$ is directory node, $T_{A}, T_{B}, T_{C}$ is subtrees of $\mathrm{P}$ (in figure 6.11(a)), $T_{A}$ and $T_{C}$ are the neighbor subtrees of $T_{B}$. As a result of $\mathrm{V}$-split for node $\mathrm{B}$, node $\mathrm{D}$ is created and replaces the node $\mathrm{B}$ in its original position in the subtree $T_{P}$. Node $\mathrm{B}$ and the new sibling node $\mathrm{B}^{\prime}$ become the children of node D. $T_{D}$ is the subtree in $T_{P}$ rooted at node D.(Figure 6.11(d))

Proposition: If the root of a subtree is created from a vertical split, there cannot be any horizontal link between descendants of the root and the nodes in neighbor subtrees. In the case of Theorem 1, there will not be any horizontal link between the nodes in $T_{D}$ and the nodes in $T_{A}$ and $T_{C}$.

Proof: If the root of the subtree is created from a recent vertical split, it has only two children. In figure $6.11(\mathrm{~b}), \mathrm{D}$ is the root caused by V-Split of original node B, and its children is the modified node $\mathrm{B}$ and new node $\mathrm{B}^{\prime}$. Initially the depth of $T_{D}$ is 3 , because $\mathrm{B}$ and $\mathrm{B}^{\prime}$ only have data nodes as children. The depth of original Node B is increased by 1 . It was proved in Theorem 1 that the horizontal links from neighbor subtrees to/from B and $\mathrm{B}^{\prime}$ can be safely removed. In addition, it is shown in Lemma 1 that there is no need to create new horizontal links between $\mathrm{D}$ and neighbor subtrees $T_{A}$ and $T_{C}$. Therefore, no horizontal 
links can be created to/from $T_{D}$ to A and C.

The width and depth grows while more nodes in $T_{D}$ having horizontal and vertical split. To prove that no horizontal link is created to/from neighbor subtrees during every vertical and horizontal split, the following cases can be considered.

Assume $\mathrm{d}$ is the depth of subtree $T_{D}$. For each level i, $1<=i<=d$, in subtree $T_{D}$ :

Case 1: In case of a horizontal split in $T_{D}$, the level becomes wider by the horizontal split, however it does not get connected to the neighbor subtrees. The horizontal links are created only within $T_{D}$. Therefore, a horizontal split does not create horizontal links to the neighbor branches at any level i. This case is illustrated in figure 6.11(c).

Case 2: In case of a vertical split in $T_{D}$, as proved in Theorem 1 and Lemma 2, the new node created from the split, does not create horizontal links to/from any sibling node. Therefore, it does not create any horizontal link to neighbor subtrees in level i. In addition, children of this node are moved down to level $i+1$ in the tree and remove their horizontal links to/from sibling nodes of their new parent in level i. Therefore, no horizontal link will be created to neighbor subtrees by a vertical split at levels i and $i+1$. This case is illustrated in figure 6.11(e).

In both cases above, we showed that if $T_{D}$ becomes wider or deeper by 1 level, no horizontal link is created to/from sibling nodes of D (i.e. A and C). In general, as subtree $T_{D}$ becomes deeper from depth i to $\mathrm{i}+1$, no horizontal link is created to/from sibling nodes of $\mathrm{D}$ in depth $\mathrm{i}$. This can be applied to all levels: For every level $\mathrm{i}$ in $T_{D}, 1<=i<=d$, no horizontal link is created to/from any nodes of neighbor subtrees $T_{A}$ and $T_{C}$. New horizontal links are only created within the new descendants inside subtree $T_{D}$.

According to Theorem 1 and 2, when V-split happens to a directory node, the subtree rooted at the new node created by this $\mathrm{V}$-split becomes independent. The independent subtrees can be the candidates for migrations. The details will be described later.

\subsubsection{The network communication Structure and message serialization}

The distributed PDCR tree allows data to be distributed among a master and workers. Clients send data insertions and query requests to the master node. Then the master takes the corresponding actions and decides if any further actions should be executed in some 


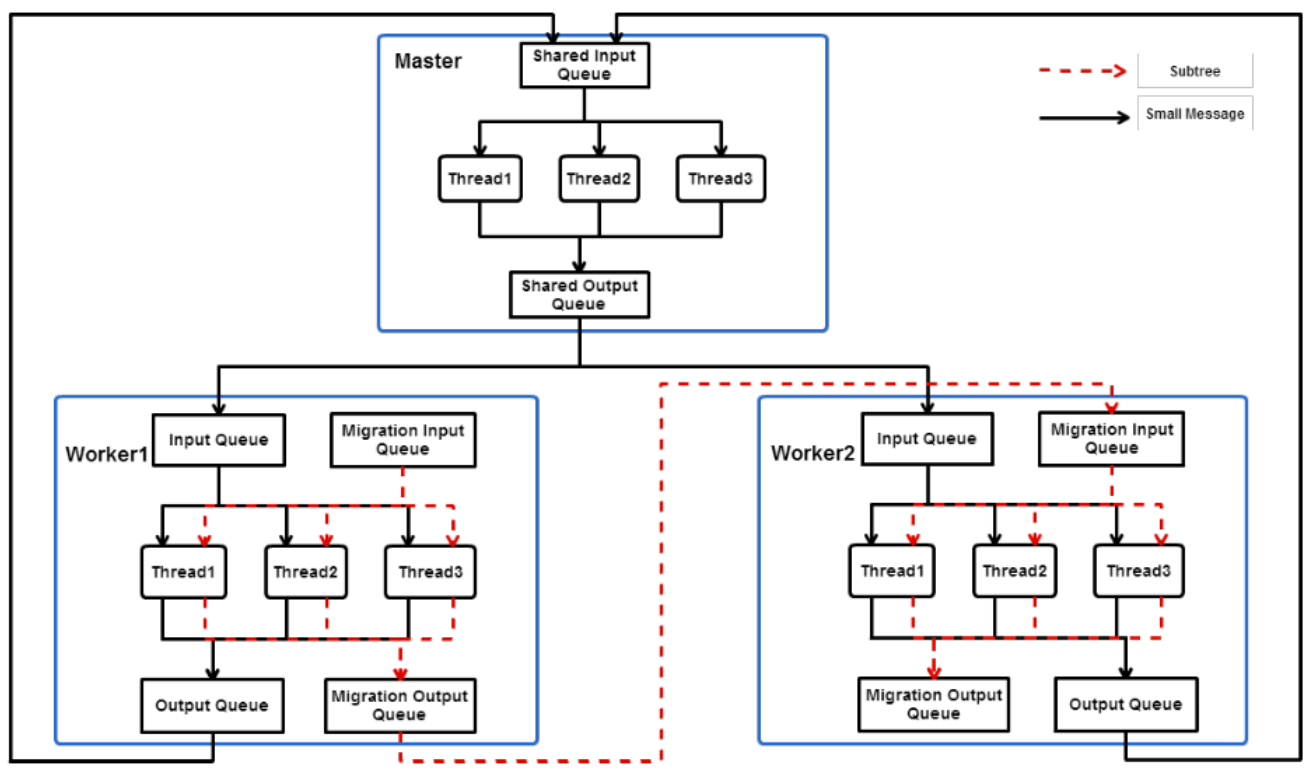

Figure 2: Global Architecture of Network Communicator

Figure 6.12: Global architecture of network communicator

workers. If so, the master will send messages and data to the selected workers to trigger the actions on the workers. Once the workers receive the messages, they can take actions correspondingly. The workers also send messages or data to the master or other workers to provide responses and replies.

The messages are passed within network by the ZeroMQ which is a high-performance messaging library but acts like a concurrent framework [1]. The ZeroMQ can create sockets on each node and provide message queues for asynchronous messages passing between different sockets. It has no single bottleneck of network since it does not use message broker. Messages can be queued if there is no peer available to receive them. Unlike conventional sockets who only allow peer to peer, one-to-many and many-to-one relationships, the zeroMQ supports the many-to-many communication pattern. With the transport flexibility, a master can send/receive messages to/from workers and workers can not only reply the master but also communicate among workers. Figure 6.12 demonstrates the architecture that is used in our design.

On the master node, the ZeroMQ opens a socket for shared input and output queues. The messages (e.g "initialize subtree", "request migration", "query message") are pushed 
into a shared output queue. The ZeroMQ picks up the messages from the output queue and send them to the workers.

On the worker node, the ZeroMQ also opens one socket for input and output queues and one socket for migration input and output queues. When messages arrive, they are pushed into the input queue for processing. Any free threads on workers can get messages from the input queue and decide the next actions based on the message received. When the workers need to respond the master or other workers, the messages are pushed into the output queue so that the ZeroMQ can sent them to destination nodes over the network. During a migration, a large subtree can be compressed as a single string message to be sent to its destination worker. The messages containing the migration subtrees are sent through the migration input/output queues on workers instead of the regular shared input/output queues. Therefore, other small messages would not be blocked by the large migration messages.

ZeroMQ's transport is message-based instead of stream-based, hence, it can transfer well-delimited chunks of data rather than raw stream of bytes. However, it usually sends string or binary types data and doesn't know anything about the data to be sent except the data size in bytes. Therefore, a message serializer is required to convert the complex data structure into strings which can be sent by the zeroMQ. On the destination nodes, the received strings are required to be de-serialized and recovered to their original formats. Besides sending simple messages through a network, any subtree of the PDCR tree can also be transferred from one node to another for a data migration purpose. We implemented a message serializer with the Boost Serialization Library. For any data objects (e.g. subtree, DirectoryNode, DataNode), the serializer goes through each member in the objects, converts each of them to a string and adds them up to one big string. There are delimiters added during serialization by the Boost library in order to identify the members. The serialized messages are wrapped by the zeroMQ and pushed into message queues. When a node receives a message through zeroMQ, the de-serializer will be called to unpack the message. Based on the delimiter the string can be reconstructed to its original data structure. 


\subsubsection{Migration process and load balancer}

Migration is a mechanism to reduce the load of a worker that is experiencing a high load such as high memory usage or cpu usage. When the performance is slow due to the high load on a worker, a migration process is triggered to move one of subtrees to another worker having a lower load in the system. In the previous proof of independent subtrees, we know a subtree whose root is created from a V-split has no links with its neighbor subtrees. The independent subtrees can be the candidates to be moved between nodes and only parent links of the root of a migration subtree need to be maintained.

The load balancer runs periodically (e.g. every few minutes) to check the memory usage on each node. Once the load balancer detects a worker having memory usage exceed a threshold, it will trigger the migration. The process choses a subtree (called migration subtree) and moves it from one worker (called source worker) to another worker (called destination worker). The migration process can also be triggered when the initial subtrees on a worker become independent. By doing that, the subtrees are distributed to multiple workers so that the work load can be potentially spread out between workers. (see figure 6.9(c) - (e)in the previous section 'Building distributed PDCR-tree from master to worker').

The migration process sends a migration subtree to a destination worker. The migration subtree is a snapshot of the subtree at the time point when migration starts. To achieve this, we could choose to lock the whole subtree to perform migration. However, when a subtree is very large, the insertions can be interrupted by the lock for a long period of time till the migration is completed. Instead, our method allows insertions to be still performed on the migration subtree in the source worker during a migration and all the queries who traverse the migration subtree can still include the most recent new data. Once a migration started, if a directory node A needs to be updated, a copy of the node A will be stored to "Links_to_backup" before any update is made. The details are showed in Algorithm 1 Node_Backup. The node update can be any type of updates including MDSR update, measure update, node split and inserting a new data node under a directory node. If a directory node has never been updated during a migration, the copy is not necessary to be made. Creation of the backup for a directory node is done at the first update and is only performed once since we only need one backup of a node to preserve its old structure before the migration. 


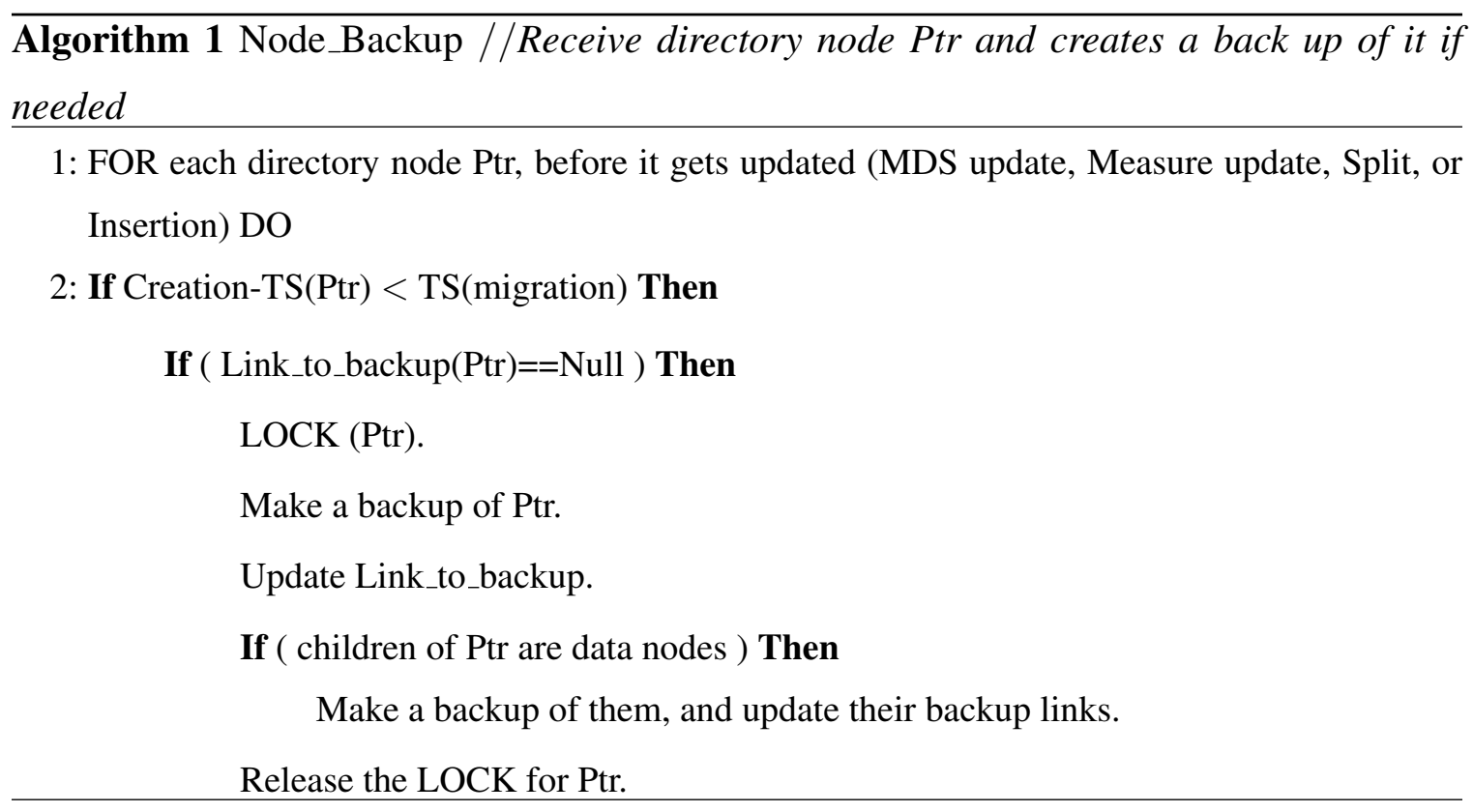

When migrating the subtree from one worker to another, for those directory nodes/data nodes having no update, they are directly copied over to the destination worker; for those nodes having been updated, their copies stored in the "Links_to_backup" shall be transferred to the destination worker. After the migration of a subtree is completed, in the destination worker we apply the same insertions that were sent to the subtree in the source worker during the time of migration, therefore, the migrated subtree in the destination can include the new data coming into the system during a migration and be ready for taking new queries. Figure 6.13 demonstrates the migration process.

Step 1: Once a migration is triggered, a master node selects a subtree on the source worker to be migrated (the subtree is called migration subtree) and also a destination worker for a migration in the Round-robin fashion. Then it will send a request to the destination worker.

Step 2: The destination worker receives the message and initializes a subtree, so the root of the subtree will be linked to the parent node of a migration subtree. After initialization, it sends a message to notify the master node that it is ready for the migration and also starts to check the migration input queue in order to receive the subtree from the queue. 


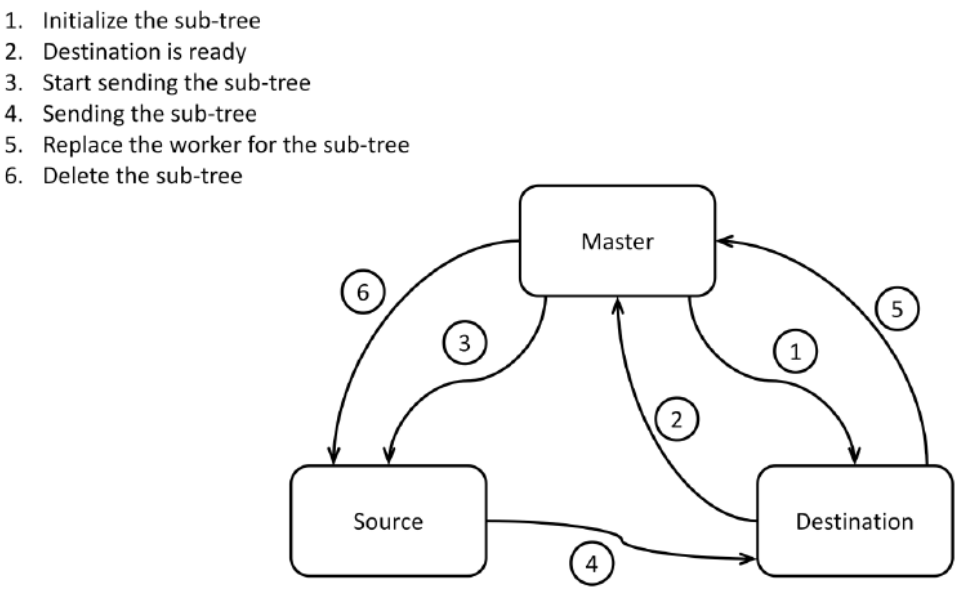

Figure 6.13: Subtree migration process

Step 3: The master node sends a message to the source worker to start the migration after it gets the "ready" message from the destination worker. Upon this message, the master node starts to send any insertions which need to traverse the migration subtree to the input queues on both the source worker and the destination worker. Therefore, insertions can still be executed on the migration subtree after the migration is kicked off and the same insertions are waiting in the queue on the destination worker till the subtree copy from the source worker to the destination worker is finished.

Step 4: The source worker sets up the migration time stamp once a migration starts up and it starts sending the migration subtree over to the destination worker. On the destination worker, the migration input queue will be checked for receiving all directory nodes and data nodes of the migration subtree. The received nodes are stored under the root of the subtree initialized in Step 2. During the process of copying the nodes from the source worker, all insertions and queries are still being executed in the source worker.

Step 5: When the destination worker receives all the nodes of migration subtree, the insertions queued in the input queue will be executed so that the subtree is updated with all new data coming into the system after the migration starts. Then, the destination worker notifies the master node that the migration copy is completed. 
Step 6: Once the master node gets the message of migration copy completion, it can start sending all new insertions and search queries to this subtree on the destination worker as usual and no longer sending requests to the source worker anymore. Furthermore, the master node will send a message to the source worker to delete the migration subtree. On the source worker, when all active insertions and queries, which are waiting in the input queue or running on this subtree, are completed, the migration subtree will be deleted.

\subsection{CR-OLAP operations and algorithms}

The distributed PDCR tree is able to handle operations like Insertions and Aggregations. An insertion operation is to add a new data into the system; An aggregation operation includes SUM, MAX, MIN, MEAN, AVG etc. For an aggregation operation, the system reports the results to the client. When clients send any requests to the system, these operations are queued in the shared input queue on the master node. The Task Assignment (Algorithm 2) is invoked to dispatch the requests to different processes. The corresponding

processes all start from the master node and then the tasks are distributed to the related worker nodes. When each worker completes its task and sends back the results, the master node will add each worker's result together and reply it to the client.

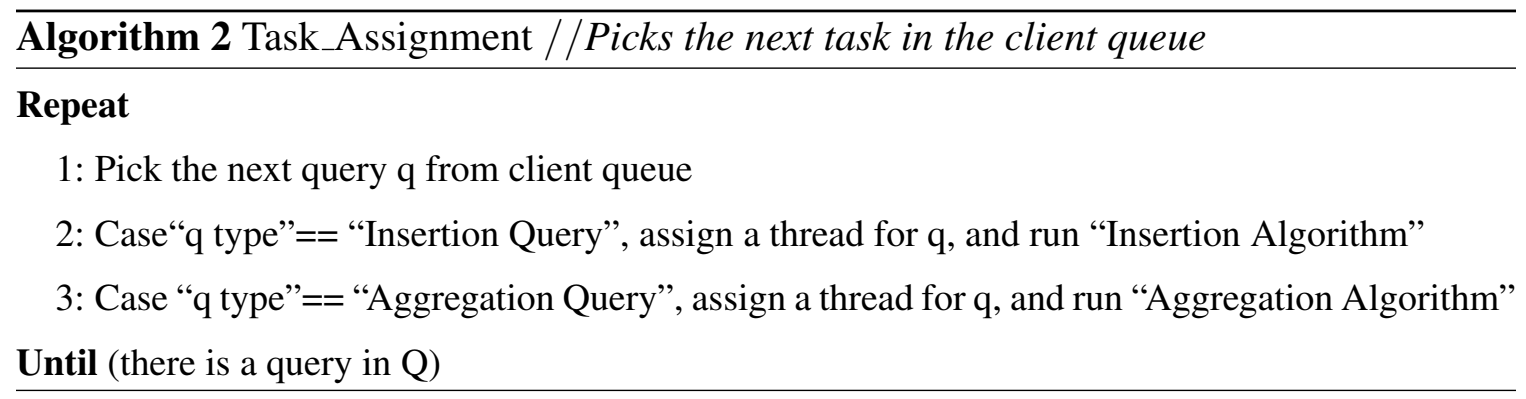

\subsubsection{Insertion}

The new data node to be inserted contains a set of MDSs. The Insertion_Master (Algorithm 3) traverses the hat from the root of the PDCR tree on a master node and checks if the MDSs of the new data node is in the MDSRs of each directory node. At each directory node, three cases may occur. 
- 1) If new data MDS is contained in the MDSR of only one of a directory node's children, the process go to the child node for further process (step 2);

- 2) If new data MDS is contained in the MDSR of more than one of a directory node's children, then the process select the child node whose subtree has the least number of data nodes (step 3);

- 3) If new data MDS is not contained in any MDSR of a directory node's children, then it should chooses the node which will cause minimum MDS overlap with other children (step 4).

The step 4 will be performed on a copy of a directory node in order to decide which node should be choose. Step 1 to 4 will be repeated till a leaf node is reached. In the hat, leaf node can be either a directory node or a data node. If it is a data node, then insert the new data and trace the path back to the root to update the MDSs/MDSRs and the measures of each directory node on that path (step 5); if it is a directory node which stores the parent of a subtree on the worker, the insertion request will be sent to the worker where the subtree is stored and continue the insertion process on the worker node (step 6). Meanwhile, if the subtree is in the process of a migration, a copy of the insertion request will be also sent to the destination worker for processing after the migration is completed. On the worker node, when it receives the insertion request, the Insertion_Worker (Algorithm 4) will be invoked and travel down to the subtree. The process is similar to the process on a master node.

During step 5 of a insertion, the directory node capacity will be checked and if the capacity is exceeded, a horizontal split process Split_Bubbleup (Algorithm 5) will be invoked. The horizontal split creates a new directory node Ptr' as the right sibling of the original directory node Ptr (step 3,4), and distributes the children nodes as well as the new data between the new directory node and the original directory which will lead to maximum MDS coverage within one directory node and minimum MDS overlap between the two directory nodes. The new directory node takes the time stamp of the original directory node and the original directory node gets the new time stamp (step 5). On the worker node, if the subtree is in processing migration, the Node Backup (Algorithm 1) will be called to make a copy of the original directory node before any updates for the migration purpose. These 
steps are started from the bottom of the tree and can be repeated until reach a parent node whose capacity is not exceeded. The PDC minimal locking scheme is still carried in this design. Therefore, at most two nodes, the current node and its parent node, are locked for processing at any point in time. This should not cause significant waiting time for parallel transactions.

If the capacity for each directory node up to root node is all reached, then a vertical split will be processed (Algorithm $6 \boldsymbol{V}$ Split). The vertical split first performs a horizontal split (step 2 to Step 6) and then it creates a new directory node D to replace the place where the original directory node Ptr is in the tree. Ptr and Ptr' becomes the children of D. To be noticed, the link to Ptr and the link from Ptr' are not necessary any more as we proved in section 6.2 so they are removed. In addition, the vertical split should also revoke the Node_Backup in order to back up the current node and its parent node if the node is in a subtree that is migrating.

\subsubsection{Aggregation}

An aggregation operation takes a range of multiple dimensions and returns the aggregated value of measures (e.g. total amount of sales) to the customer. As same as the PDC-tree on multi-core processors, a stack is still used to track the tree traversal and to detect the new data inserted to the system during the aggregation process. The Aggregation_Master (Algorithm 7) starts from the root of the hat on a master node and pushes directory nodes into the stack $\mathrm{S}$ (step 2). When the directory node in $\mathrm{D}_{\text {stack }}$ is popped from $\mathrm{S}$, the process will find the corresponding directory node $\mathrm{D}$ in the tree and compare the time stamps (step 3). If the time stamp of $\mathrm{D}_{\text {stack }}$ is earlier than the time stamp of $\mathrm{D}$, it means one or more new data that are inserted into the subtree of $\mathrm{D}$ or its neighbour subtrees. The process traverses all right sibling nodes of $\mathrm{D}$ whose timestamp is later than or equal to the timestamp of $\mathrm{D}_{\text {stack }}$ through "right sibling link" of each directory node and push them into the stack for further process. Therefore, the newly inserted data can be evaluated for this query. At each directory node (step 3.3), the MDSs of a range query and the MDSRs of a directory node will be adapted to the same level if it is necessary. For example, if a directory node contains MDSR at city level and the range query contains MDS at Country level, the MDSR in directory node should be adapted to Country level. We can simply set '0' to the lower level 
sub-ids in the MDS Ids to retrieve a higher level MDS Ids (Chapter 6, Section 6.2:MDS and MDSR) If the MDSRs of the directory node is included in the MDSs of the range query, the measures will be added to the result; if the MDSRs of the directory node is not fully contained in the MDSs of the query range, there are two cases depending on the types of leaf nodes in the hat. If it is not a directory node that points to subtrees, the directory node will be pushed into the stack, otherwise, the algorithm finds which worker stores the subtree and sends the aggregation query to that worker to continue searching following the similar steps in the Aggregation_worker (Algorithm 8). The process repeats the steps until the stack is empty and then returns the final aggregation result. 
Algorithm 3 Insertion Algorithm on the master //insertion of data node d in distributed PDC-tree X

1: Start from R (the root of the hat), and set Ptr point to R.

\section{Repeat}

2: If MDS(d) is contained in the MDSR of only one of the children of Ptr Then set Ptr equal to the directory node for that child.

3: If MDS(d) is contained in the MDSR of more than one of the entries of Ptr Then set Ptr equal to the root of the child sub-tree with minimum number of data nodes.

4: If MDS(d) is not contained in any MDSR of a child of Ptr Then

a. Make a copy Ptr' of node Ptr.

b. For each child C of Ptr': Add the new data item d to C and calculate the MDS enlargement and overlap caused.

c. Set $\operatorname{Ptr}=$ the child which causes minimal overlap.

UNTIL Ptr is a leaf node in the hat

5: If (Ptr is pointing to a data node) Then

a. Set Ptr=parent of Ptr.

b. Acquire a LOCK for Ptr.

c. Insert data item d into Ptr and update the measure(s), MDS(Ptr), and time stamp of Ptr i.e. TS(Ptr).

d. If capacity of Ptr is exceeded Then

1. WHILE $(\mathrm{Ptr} !=\mathrm{R}) \& \&$ (capacity of Ptr $>=$ Cap) set Ptr=Parent of Ptr.

\section{If $(\operatorname{Ptr}==\operatorname{Root})$ Then}

a. Call V_Split for the parent of $d$, and create a new node Y.

b. If (Depth of $Y==$ Cut) Then

1. (Counter ++ , assign counter to the new sub-tree)

2. select next worker using load balancing strategy

3. Send children of $Y$ to the chosen worker to initiate a sub-tree in it.

Else Call Algorithm 5 (Split_Bubbleup) Until (capacity of $\operatorname{Ptr}<=$ Cap)

6: If (Ptr is pointing to a Sub-tree's number) Then

a. In worker-subtree map, find the worker, w, which stores $\mathrm{t}$

b. If $t$ is migrating, send the insert query to migrating destination worker

c. Send the insert query to $\mathrm{w}$ 


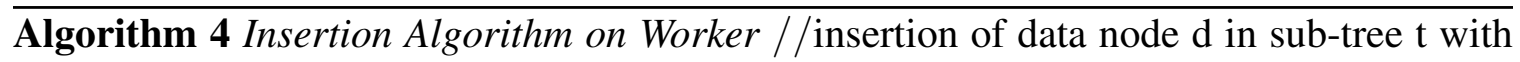
root $\mathrm{r}$

1: Start from $r$ (the root of $t$ ), and set Ptr point to $r$.

2: If MDS(d) is contained in the MDSR of only one of the children of Ptr Then set Ptr equal to the directory node for that child.

3: If MDS(d) is contained in the MDSR of more than one of the entries of Ptr Then set Ptr equal to the the root of the child sub-tree with minimum number of data nodes.

4: If MDS(d) is not contained in any MDSR of a child of Ptr Then

a. Make a copy Ptr' of node Ptr.

b. For each child C of Pt': Add the new data item d to C and calculate the MDS enlargement and overlap caused.

c. Set $\operatorname{Ptr}=$ the child which causes minimal overlap.

UNTIL Ptr is a data node

5: Set Ptr=parent of Ptr.

6: If TS(migration) is set Then Call Algorithm Node_Backup(Ptr)

7: Acquire a LOCK for Ptr.

8: Insert data item d into Ptr and update the measure(s), MDS(Ptr), and time stamp of Ptr i.e. TS(Ptr).

9: If capacity of Ptr is exceeded Then

a. WHILE (Ptr $!=r) \& \&$ (capacity of Ptr $>=$ Cap) set Ptr=Parent of Ptr.

b. If $(\operatorname{Ptr}==r)$ Then

1. sends a request to the master to check up to which level in the hat, ancestors of Ptr are full

2. If capacity of all directory nodes up to root in hat is full, Then Call Algorithm 6 V_Split for the parent of $d$.

3. If capacity is not full up to root in hat, Then Call Algorithm 5 (Split_Bubbleup) UNTIL (Ptr==Parent of $r$ (leaf of the hat))

4. Send a request to the worker queue to call Algorithm 5 (Split-bubbleup) UNTIL (capacity of $\operatorname{Ptr}<=$ Cap) in the master for Ptr.

Else Call Algorithm 5 (Split_Bubbleup) Until (capacity of $\operatorname{Ptr}<=$ Cap) 

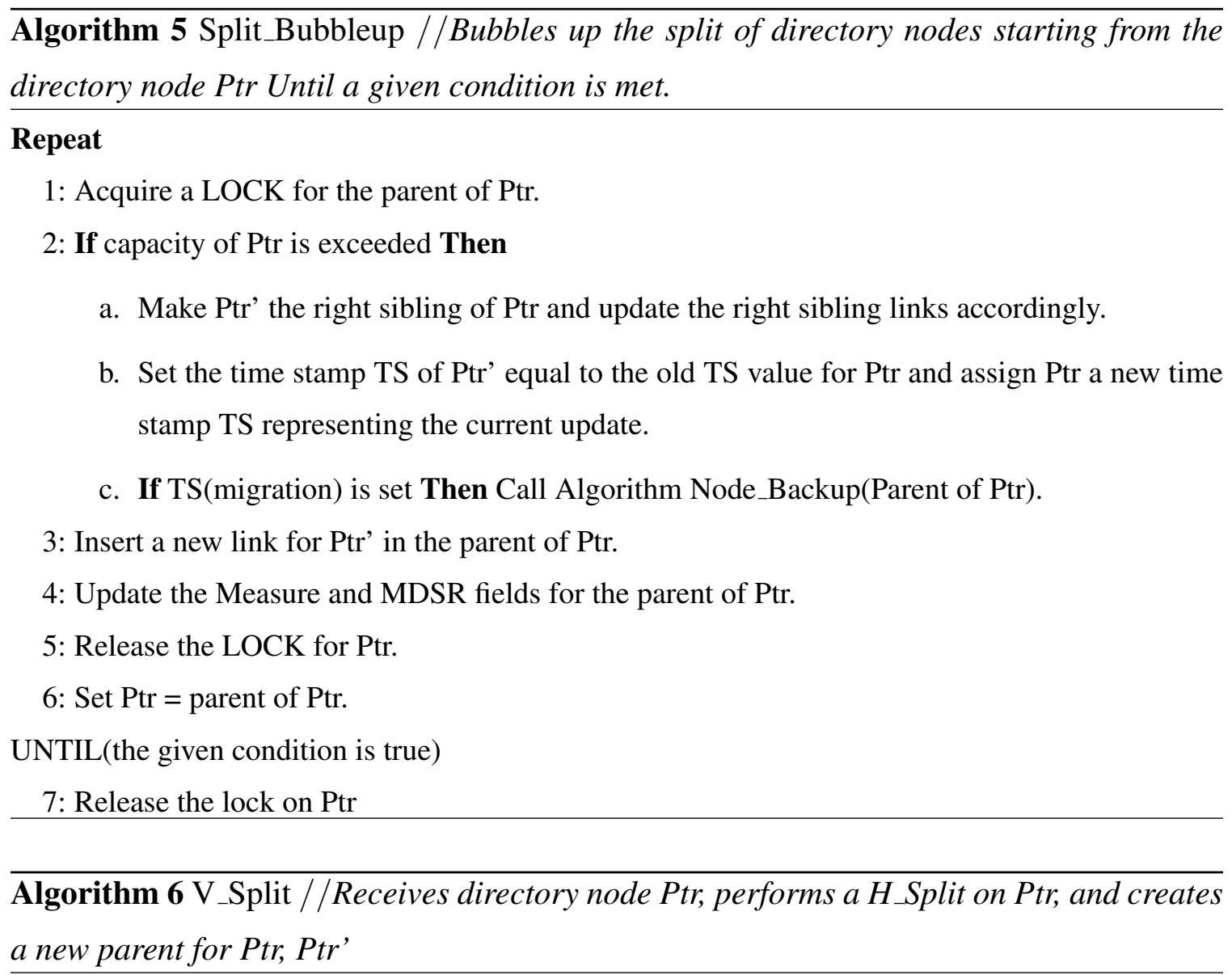

1. If TS(migration) is set Then
a. Call Algorithm 1 Node_Backup(Ptr)
b. Call Algorithm 1Node_Backup(Parent of Ptr)

2. Acquire a LOCK for Ptr and the parent of Ptr.

3. Split Ptr into two directory nodes Ptr and Ptr'(DC-tree split algorithm, sections 4.2 and 4.3).

4. Make Ptr' the right sibling of Ptr and update the right sibling links accordingly.

5. Set the time stamp TS of Ptr' equal to the old TS value for Ptr and assign Ptr a new time stamp TS representing the current update.

6. Update MDSR of Ptr and Ptr', and their measures.

7. Create a new directory node D with a new TS, Add two entries for Ptr,Ptr' in D as its children, and update MDSR(D) covers MDSR(Ptr) and MDSR(Ptr').

8. Replace the entry of Ptr in the old parent of Ptr with an entry for D.

9. Remove the Link from left sibling of Ptr to Ptr.

10. Release the LOCK for Ptr and the old parent of Ptr. 
Algorithm 7 Aggregation Query Algorithm on the Master //Compute the aggregate value
for query q in partitioned PDC-tree X

1: Set Ptr=R, Push Ptr into a stack S for query q.

\section{Repeat}

2: Pop top item from stack S, call it Ptr'.

3: If the time stamp (TS) of Ptr' is smaller (earlier) than the time stamp (TS) of Ptr Then

3.1: Using the "Link to Sibling" field in directory nodes, traverse the list of siblings of Ptr. Push all sibling nodes up to a node with its TS equal to TS of Ptr' (Push from right).

3.2: Push Ptr again into stack $\mathrm{S}$.

Else

3.3: FOR each child C of Ptr DO

3.3.1: For each dimension of $\mathrm{C}$ where $\operatorname{MDSR}(\mathrm{C})$ and range $\operatorname{MDS}(\mathrm{q})$ are at different levels in the dimension hierarchy, convert the lower level entry to the higher level.

3.3.2: If $\operatorname{MDSR}(C)$ is contained in range $\operatorname{MDS}(q)$ Then add Measure(C) to the result value.

3.3.3: If $\operatorname{MDSR}(\mathrm{C})$ overlaps range $\operatorname{MDS}(\mathrm{q})$ but is not contained in it, Then

If ( $\mathrm{C}$ is pointing to a sub-tree) Then Send the Aggregation Query to the worker Counter++

Else Push C into stack S UNTIL stack S is empty.

4. IF query is distributed to workers Then

Wait all queries are finished on workers

Calculate the aggregated results.

5. Report the aggregation value to client. 


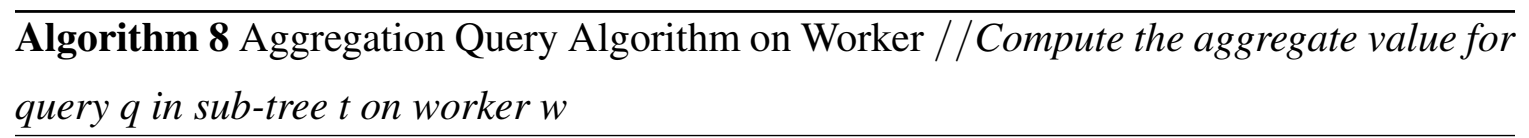

1: Set Ptr=r, Set result value Total=0, Push Ptr into a stack $S$ for query q.

\section{Repeat}

2: Pop top item Ptr' from stack $S$.

3: If the time stamp (TS) of Ptr' is smaller (earlier) than the time stamp (TS) of Ptr Then

3.1: Using the "Link to Sibling" field in directory nodes, traverse the list of siblings of Ptr. Push all sibling nodes up to a node with its TS equal to TS of Ptr' (Push from right).

3.2: Push Ptr again into stack S.

\section{Else}

\section{3: FOR each child C of Ptr DO}

3.3.1: For each dimension of $\mathrm{C}$ where $\operatorname{MDSR}(\mathrm{C})$ and range $\operatorname{MDS}(\mathrm{q})$ are at different levels in the dimension hierarchy, convert the lower level entry to the higher level.

3.3.2: If $\operatorname{MDSR}(\mathrm{C})$ is contained in range $\operatorname{MDS}(\mathrm{q})$ Then add Measure(C) to Total.

3.3.3: If $\operatorname{MDSR}(\mathrm{C})$ overlaps range $\operatorname{MDS}(\mathrm{q})$ but is not contained in it, Then Push C into stack S UNTIL stack S is empty. 


\subsection{CR-OLAP query types}

The PDCR tree is designed to answer a set of queries in parallel. For a fact table with $\mathrm{d}$ dimensions in a data warehouse, the set of queries $\mathrm{Q}$ is defined as $\left\{q_{1}, q_{2}, q_{3}, . . q_{d}\right\}$ where $1 \leq \mathrm{i} \leq \mathrm{d}$, and $q_{i}$ is a set of values to be searched in dimension $\mathrm{i}$. The set of values in $q_{i}$ can be represented in the following formats:

1. Multiple ranges of values covering a contiguous range of values in a dimension $i$.

This type of query value is used only for ordered dimensions such as date, time, etc. For example, assume we have an ordered date dimension with the concept hierarchy Year-Month-Day, a query containing a set of values $\left\{\left[2011-*_{-} *^{-}, 2013-*_{-} *\right]\right\}$ means date from 2011 to 2013. To use MDS ids to represent this query, it becomes $\{[2011-$ $0-0,2013-0-0]\}$. The 0 s in the ids can be interpreted as * that we usually have it in SQL queries and it means All.

2. Multiple MDS Ids at any level of the hierarchy of a dimension i.

Each MDS Id in a hierarchy level $l$ in dimension i covers many distinct MDS Ids in the level $l+1$ of the dimension. This type of query values is used for both ordered and unordered dimensions such as Location, Product, Date, etc. For example, assume we have a store dimension having the hierarchy Counry-Province-City-StoreId, a query containing a set of values $\{$ Alberta, Ontario $\}$ means all stores located in the provinces of Alberta and Ontario. The MDS Ids that represent the query is \{Canada-Alberta-00, Canada-Ontario-0-0 $\}$. In the implementation, 'Canada', 'Alberta', 'Ontario' and ' 0 ' is presented in total 64-bits integer as we introduced in section 6.2

There are two types queries that the CR-OLAP system can support to answer. One is a range query and the other one is a point query.

1. Range queries for a dimension $\mathrm{i}$ :

$\mathrm{q}_{i}=\left\{\left[\right.\right.$ low $I D_{i 1}$, high $\left.I D_{i 1}\right],\left[\right.$ low $I D_{i 2}$, high $\left.I D_{i 2}\right], \ldots$, [low $I D_{i n}$, high $\left.\left.I D_{i n}\right]\right\}$, where low $I D_{i j}$ represents the lower bound of the $j$ th given range in dimension $\mathrm{i}$, and high $I D_{i j}$ represents the upper bound of the $j$ th given range in a dimension $i$. 
2. Point queries for dimension i:

$\mathrm{q}_{i}=\left\{I D_{i 1}, I D_{i 2}, \ldots, I D_{i n}\right\}$, where $I D_{i j}$ represents the $j$ th given MDS ID in dimension i. Note that IDs can be in different levels of dimension $i$.

Figure 6.14 lists a few examples.

\begin{tabular}{|c|c|c|c|c|}
\hline $\bar{Q}$ & $\begin{array}{l}q_{1} \text { (Store dim) } \\
\text { Country- } \\
\text { Province-City }\end{array}$ & $\begin{array}{l}q_{2} \text { (Date dim) } \\
\text { Year-Month- } \\
\text { Day }\end{array}$ & Query MDS ids & Description \\
\hline 1 & Canada & $\begin{array}{l}\text { [2009-***, } \\
\text { 2011-*-*] }\end{array}$ & $\begin{array}{l}\{\{\text { Canada- } 0-0\}, \\
\{[2009-0-0,2011-0-0]\}\}\end{array}$ & $\begin{array}{l}\text { All stores in Canada } \\
\text { from } 2009 \text { to } 2011\end{array}$ \\
\hline 2 & $\begin{array}{l}\text { Ontario, Alberta, } \\
\text { Quebec }\end{array}$ & *-January-* & $\begin{array}{l}\{\{\text { Canada-Ontario- } 0-0, \\
\text { Canada-Alberta-0-0, } \\
\text { Canada-Quebec-0-0 }, \\
\{0 \text {-January- } 0\}\}\end{array}$ & $\begin{array}{l}\text { Stores in } \mathrm{ON}, \mathrm{AL} \text {, or QC } \\
\text { in January of all years }\end{array}$ \\
\hline 3 & US, Quebec & 2010-Feb-* & $\begin{array}{l}\{\{\text { US- } 0-0-0, \\
\text { Canada-Quebec- } 0-0\} \\
\{2010-\text { Feb- } 0\}\}\end{array}$ & $\begin{array}{l}\text { Stores in the US or } \\
\text { Quebec in the first day } \\
\text { of every month }\end{array}$ \\
\hline 4 & Ottawa, Toronto & $\begin{array}{l}\text { *-December-*, } \\
\text { *-September-" }\end{array}$ & $\begin{array}{l}\{\{\text { Canada-Ontario-Ottawa- } 0 \text {, } \\
\text { Canada-Ontario-Toronto- } 0\} \text {, } \\
\{0 \text {-December- } 0, \\
0 \text {-September- } 0\}\end{array}$ & $\begin{array}{l}\text { Stores in Ottawa or } \\
\text { Toronto in Dec or Sept } \\
\text { of every year }\end{array}$ \\
\hline 5 & Canada, US & $\begin{array}{l}{[2008-1-1,} \\
2008-8-31]\end{array}$ & $\begin{array}{l}\{\{\text { Canada-0-0-0, US-0-0-0 }\} \\
\{[2008-1-1,2008-8-31]\}\end{array}$ & $\begin{array}{l}\text { Stores in Canada or US } \\
\text { from Jan } 1_{\text {st }} 2008 \text { to } \\
\text { Aug } 31_{\text {st }} 2008\end{array}$ \\
\hline 6 & $*$ & 2013-June-2 & $\{\{$ All-0-0-0-0 $\},\{2013-J$ une- 2$\}\}$ & $\begin{array}{l}\text { All stores everywhere } \\
\text { in June } 2 \text { nd } 2013\end{array}$ \\
\hline
\end{tabular}

Figure 6.14: Examples of query patterns

\subsection{Summary}

The CR-OLAP is a parallel real-time OLAP system designed for cloud based distributed systems. The key component is the distributed PDCR-tree which is an extension of the PDC-tree. Using the hierarchy of dimensions, the PDCR-tree can group the data at different hierarchy levels. The data set is partitioned and distributed on multiple cloud machines so that any insertions and aggregations operations can be executed in parallel. The algorithms are given to illustrate the parallel operations. The MDS ids are modified to bit 
representation in order to improve the system performance. A MDSR (the range of MDS ids) is used for describing directory nodes so that unordered dimensions can also be expressed in an ordered manner. This helps to reduce the system response time. Meanwhile, the CR-OLAP can handle range queries at any hierarchy level as well as point queries. In next Chapter, we will demonstrate its performance. 


\section{Chapter 7}

\section{Experiment and Testing of CR-OLAP}

\subsection{Introduction}

The system is implemented in $\mathrm{C}++$. The network communicator, the message serializer and the PDCR-tree are developed as separate APIs. The ZeroMQ library is used for the network communicator and the boost serialization library is used for the message serializer. We conducted a large amount of tests to measure the system response time and throughput. The tests are performed on the Amazon cloud environment. We also developed a StreamOLAP system using One-Dimensional Index which is a linear array structure to handle data insertions and search queries in parallel. We demonstrate that the CR-OLAP system is efficient by comparing it against the Stream-OLAP system. We will introduce the details in the following section.

\subsection{Test settings}

\subsubsection{Experimental environment}

On the Amazon cloud, there are various types of instances available. We selected the instance of $\mathrm{m} 2.4$ xlarge for the master node and the M3.2xlarge instance for the worker nodes. The M2.4xlarge is an optimized instance for memory-intensive applications. It contains $68 \mathrm{G}$ memory and 8 virtual CPUs which has total 26 elastic computing units(ECU). Each ECU provides the equivalent CPU capacity of a 1.0 - $1.2 \mathrm{GHz} 2007$ Opteron or 2007 Xeon processor. The M3.2xlarge instance also has 8 virtual CPUs but it only has 30G memory size. The CC2.8xlarge instance and the CR1.8xlarge instance are claimed as cluster instances. The cluster instances can be built in one network which could have

10G network speed. They are 32 cores with hyper threading enabled and also have high memory storages. The details are listed in the Table 7.1.

We also performed experiments on the Ontario Research and Education VCL cloud. 
Table 7.1: Specifications of Amazon cloud instances used in our experiments [2]

\begin{tabular}{lllllll}
\hline \hline Instance Family & Instance Type & CPU Arch & vCPU & ECU & Memory(GiB) & Network \\
\hline Memory optimized & m2.4xlarge & 64-bit & 8 & 26 & 68.4 & High \\
General purpose & m3.2xlarge & 64-bit & 8 & 26 & 30 & High \\
Compute optimized & cc2.8xlarge & 64-bit & 32 & 88 & 60.5 & 10 Gigabit \\
Memory optimized & cr1.8xlarge & 64-bit & 32 & 88 & 244 & 10 Gigabit \\
\hline
\end{tabular}

The instance we choosed has 16 cores, 32G memory, $2199 \mathrm{MHZ}$ cpu.

A Linux operating system CentOS 6.3 is installed on each instance with the GNU GCC 4.7 compiler. It supports OpenMP to handle multi-threading to parallelize processes. A ZeroMQ 3.2 is installed as message passing middleware to transfer the data or transaction instructions between machines.

\subsubsection{Experimental data}

In all experiments of the CR-OLAP, we still use the TPC-DC benchmark which was used in the experiments of the PDC-tree for multi-core processors. The underlying business model of the TPC-DS is a retail product supplier. We select the "Store_Sales" fact table which is the largest fact table among all seven fact tables. The store_sales fact table contains 8 dimensions and several measures such as quantity, net paid, net profit, etc. The graph 7.1 lists the dimensions and the hierarchy schema of each dimension that was used in the system. The 8 dimensions include item, store, customer, date_dim, time_dim, promotion, household_demographics, customer_address. In the graph, the dimensions in left side contain ordered data and the dimensions in right side contain unordered data.

Multiple tests were conducted to evaluate the time of completing insertions and query transactions using distributed PDCR-tree index. The following scenarios are selected for our experiments.

(1) Increasing number of dimension

(2) Increasing number of workers

(3) Increasing data size

(4) Increasing data coverage for queries

(5) Using combination of star (*) at different hierarchy levels for queries 


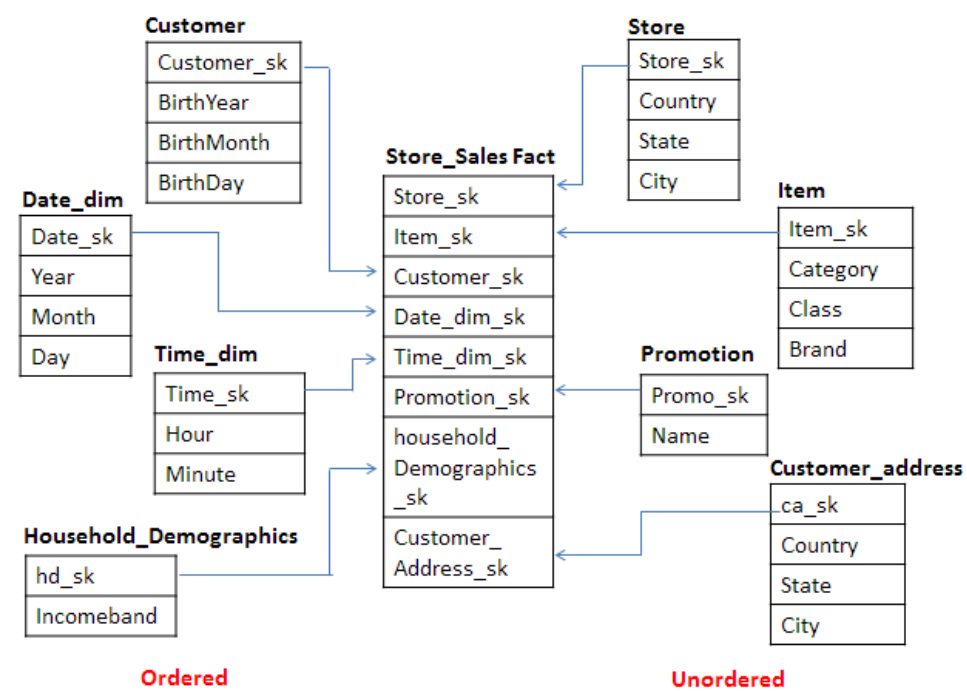

Figure 7.1: Store_Sales Fact table and 8 dimensions and each with its hierarchy scheme

(6) Running on different types of cloud instances

(7) Increasing number of cut level

(8) Increasing number of directory node capacity in master/workers

Hamed Zaboli performed the experiments of the CR-OLAP for item 1 to 5, I performed the experiments of the CR-OLAP for item 6 to 8 .

\subsubsection{Comparison baseline}

In addition, a Stream-OLAP system with a 1-dimensional array index (1D-Index) is implemented and used to be compared with the CR-OLAP system with a PDCR-tree index. Data nodes in the 1D-Index structure are as the same as they are in the PDCT-Tree index. The Stream-OLAP system creates an array for every value in the highest hierarchy level of a dimension and data nodes are stored in the related arrays according to the values in the highest hierarchy level of a dimension. For example, the 1D-Index builds an array containing all data nodes who have the value of "2012" in the highest hierarchy level "BirthYear" of the "customer" dimension. If there are 10 different values for "BithrYear" level, the Stream-OLAP creates 10 arrays for the value of each year. Data nodes in each array are not sorted and are inserted at the end of the array as they arrive. Arrays are evenly 
distributed between the workers to assure parallel processing of insertions and queries. In each array, multiple queries may search the arrays in parallel. The 1D-index structure is used to compare its performance with the PDCR-tree's performance and to evaluate the impact of a single-dimensional index versus a multi-dimensional index for hierarchical multidimensional databases. The 1D-index and the PDCR-tree are different from a B-tree and an R-tree since the later indices do not designed for the data having hierarchy structures. Mr.Kong performed the experiments of the Stream-OLAP for item 1 to 5.

\subsection{Analysis of Results}

In the following section, we will demonstrate the experiments results. All tests are performed on $\mathrm{m}+1$ machines (a single master node plus $\mathrm{m}$ worker nodes) with 16 threads on each node to concurrently process tasks.

\section{Test 1: Increasing the number of dimensions}

The test is to evaluate the impact on the number of dimensions. The master node is using an m2.4xlarge instance and all the worker nodes are using the m3.2xlarge instances. The test is performed on 8 workers $(\mathrm{m}=8)$ with 40 million tuples from the fact table $(\mathrm{N}=$ $40 \mathrm{M})$ as initial insertions followed by three sets of 1000 queries $(\mathrm{q}=1000)$. The number of dimensions $\mathrm{d}$ is increased from 4 to $8(4 \leq \mathrm{d} \leq 8)$. The three sets of queries include non-star regular queries (example 5 in section 6.4 Figure 6.14), with the coverage 10\%, $60 \%$, and $95 \%$ respectively. The coverage is measured by the percentage of the number of query values over the cardinality of the values of an attribute in a certain hierarchy level of a dimension. For example, an unordered dimension Customer_Address has 50 different values of the states in US. When the values of all queries cover 48 states, we say the coverage is $95 \%$.

Figures 7.2 and 7.3 demonstrate the results of the test. Insertions using the 1D-index is faster than they are using the PDCR-tree. This is because the 1D-index only requires inserting a new data at the end of an array while the distributed PDCR-tree has to traverse the hat and a subtree, perform comparisons on each directory node for all dimensions in the path from the root to leaf nodes as well as possibly call node splits. It is important to note that insertion time is not noticeable to system users since users usually do not require 


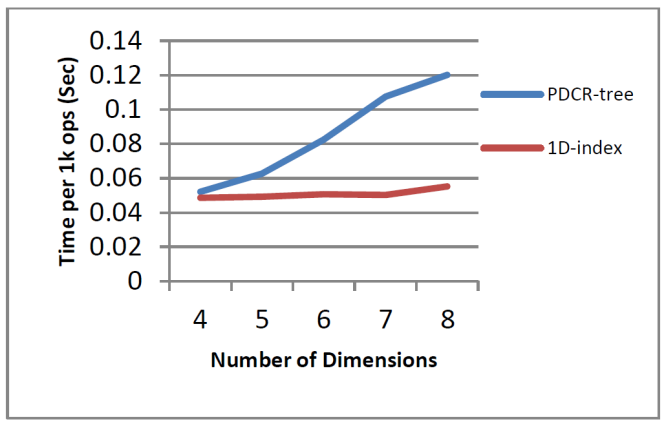

Figure 7.2: Time for 1000 insertions as a function of the number of dimensions. $(N=40 M, m=8, k=16)$

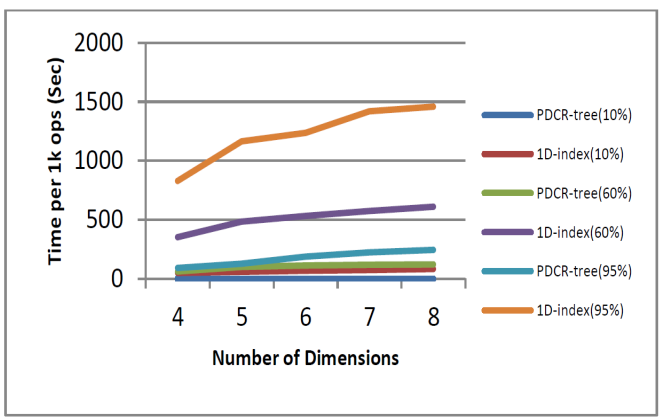

Figure 7.3: Time for 1000 queries with different query coverage, as a function of the number of dimensions. $\quad(N=$ $40 M, m=8, k=16$ )

system responses for insertions. However, the PDCR-tree handles queries significantly faster than the 1D-index does in all cases of query coverage. The query time of the PDCRtree is increased twice while the number of dimensions is doubled. The reason is: when increasing the number of dimensions, the number of MDSRs/MDSs in directory nodes is increased, therefore, the time spent on comparing MDSs of new data with MDSRs of directory nodes is increased. The test shows a reasonable performance of the PDCR-tree for handling data set with high dimensions. The PDCR-tree with 8 dimensions takes 0.12 seconds to solve a query having $60 \%$ data coverage while the $1 \mathrm{D}$-index takes 0.61 seconds that are 5 times slower.

\section{Test 2: Increasing number of workers}

The test is used to evaluate the impact by the increasing number of workers. The master node is using a m2.4xlarge instance and all worker nodes are using the m3.2xlarge instances. The test is performed on 10 million tuples from the fact table $(\mathrm{N}=10 \mathrm{M})$ with 8 dimensions $(d=8)$ as initial insertions followed by three sets of 1000 queries $(q=1000)$. The number of workers $\mathrm{m}$ is increased from 1 to $8(1 \leq \mathrm{m} \leq 8)$. The three sets of queries are no-star regular queries with coverage 10\% (low), 60\% (medium) and 95\% (high) respectively. We choose 10 million data size as the fixed data size so that it does not exceed the memory size of a single worker machine. 


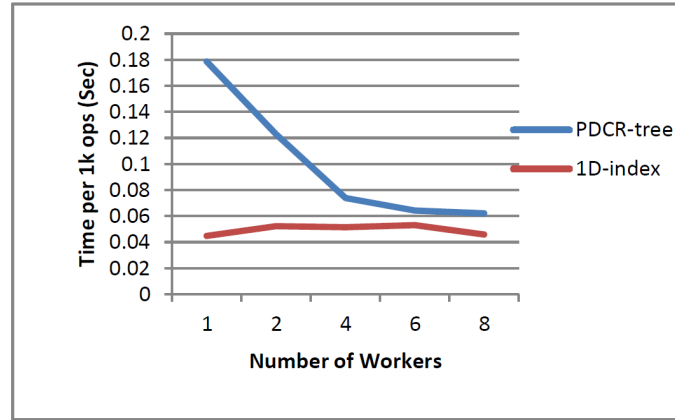

Figure 7.4: Time for 1000 insertions as a function of the number of workers. $(N=$ $10 M, k=16, d=8$ )

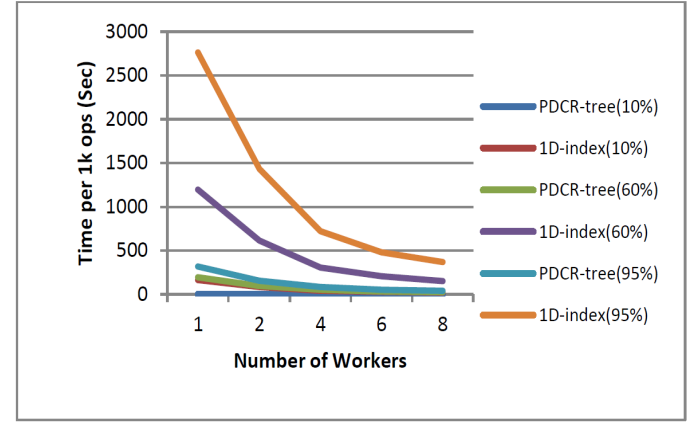

Figure 7.5: Time for 1000 queries with different query coverages as a function of the number of workers. $(N=10 M, k=$ $16, d=8)$

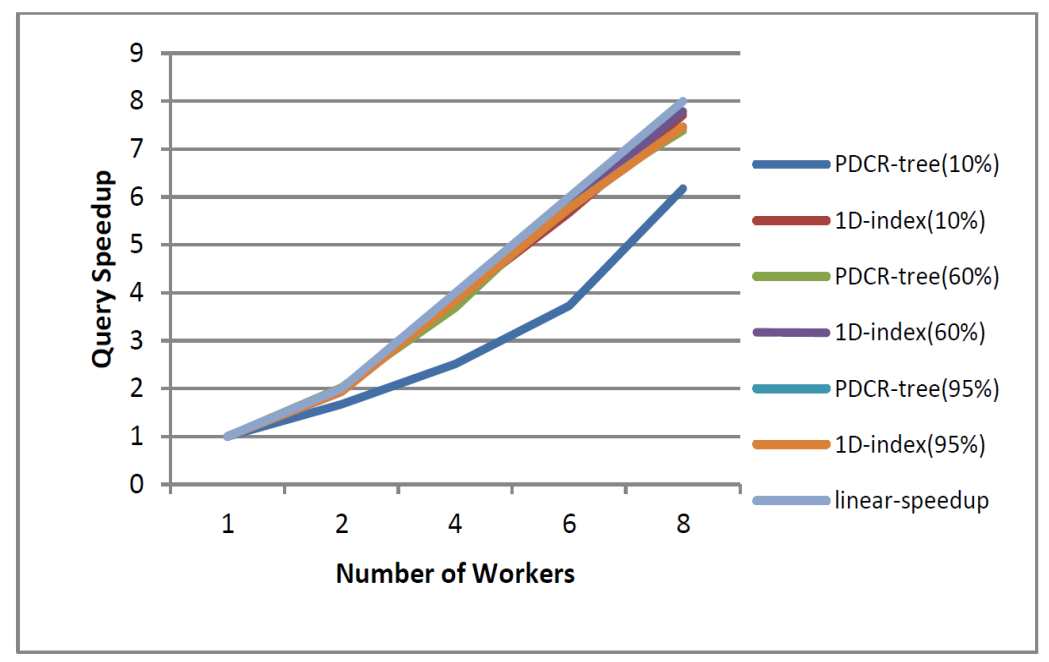

Figure 7.6: Speedup for 1000 queries with different query coverage as a function of the number of workers. $(N=10 M, k=16, d=8)$

Figure 7.4 shows the Stream-OLAP outperforms the CR-OLAP. The 1D-Index does not get speedup on insertion time. We did a test to break down the execution time of every operation involved in the Stream-OLAP and found it spent most time on data serialization and network communication. The actual data appending operation only took very little time. Each insertion requires serialization and data transportation from the master to the workers. Therefore, no matter how many workers are used, the total time on completing 1000 insertions is very close. The PDCR-tree is slower in insertions since it has overhead 
like node splits during insertions. But while increasing the number of workers, the PDCRtree insertion time is speedup since the distributed data structure allows multiple workers to process insertions concurrently. Figure 7.5 illustrates that the time for queries is decreasing by increasing the number of workers $(\mathrm{m})$. Figure 7.6 demonstrates that both the PDCR-tree and the 1D-Index achieve close to a linear speedup by increasing the number of workers. However, the PDCR-tree takes a much smaller absolute time to run queries for all cases of query coverage.

\section{Test 3: Increasing data size}

The third test is used to evaluate the performance by scaling up the size of systems. The number of worker nodes is increased from 1 to $8(1 \leq \mathrm{m} \leq 8)$ to handle the increasing data size from 10 million to 80 million tuples $(10 \mathrm{M} \leq \mathrm{N} \leq 80 \mathrm{M})$ respectively. The master node is using a $\mathrm{m} 2.4 x$ large instance and all the worker nodes are using the $\mathrm{m} 3.2 x$ large instances. The data set processed has 8 dimensions $(d=8)$ and three sets of 1000 queries $(q=1000)$ are executed after data insertions.

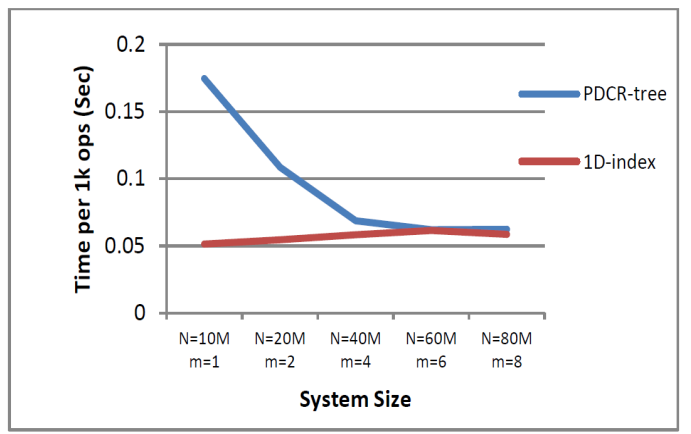

Figure 7.7: Time for 1000 insertions as a function of the data size (number of items currently stored). $(k=16, d=8)$

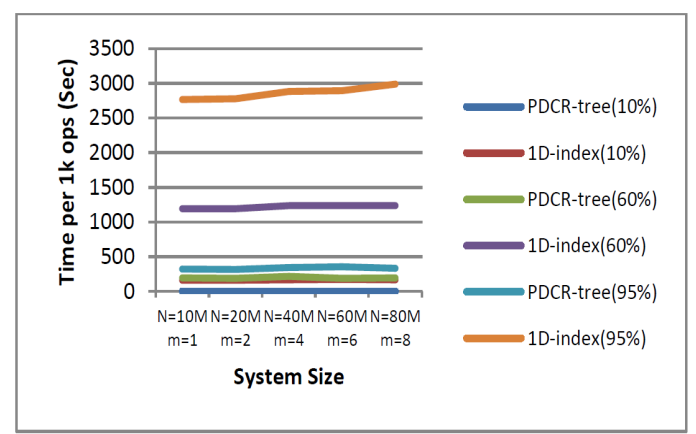

Figure 7.8: Time for 1000 queries with different query coverage as a function of the data size (number of items currently stored). $(k=16, d=8)$

From figure 7.7 and 7.8, we observe that by increasing the number of workers to process the increasing size of data the insertion time is decreased and the query performance of the system stays stable. The execution of insertions in the CR-OLAP is slower than the execution of insertions in the Stream-OLAP. However when the number of workers is increased to 8 , the total time of insertions in both systems is close. Conversely, the query 
time of the PDCR-tree is significantly faster than the query time of the 1D-index is. This test shows the system can be scaled up and process much larger data size with more number of workers without slowing down overall performance.

\section{Test 4: Increasing query coverage}

This test is used to evaluate the impact of every individual dimension on queries performance with different coverage. It was performed on 40 million tuples from the fact table $(\mathrm{N}=40 \mathrm{M})$ with 8 dimensions $(\mathrm{d}=8)$ as an initial data set. The number of workers is $8(\mathrm{~m}=8)$. We executed 9 sets of queries with the query coverage from $10 \%$ to $90 \%$ and from $91 \%$ to $99 \%$. Among the 9 sets, 8 sets have a "*” in one of the dimensions in each of them (example 3 in section 6.4 Figure 6.14) and one query set contains no-star regular queries (example 5 in section 6.4 Figure 6.14).

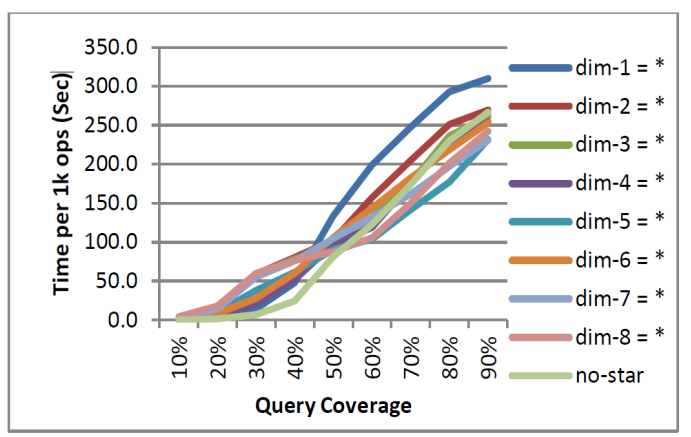

Figure 7.9: Time for 1000 queries as a function of query coverages in PDCRtree Impact of queries having value "*” for different dimensions. $(N=40 M, m=$ $8, k=16, d=8,10 \%<=$ Coverage $<=$ $90 \%)$

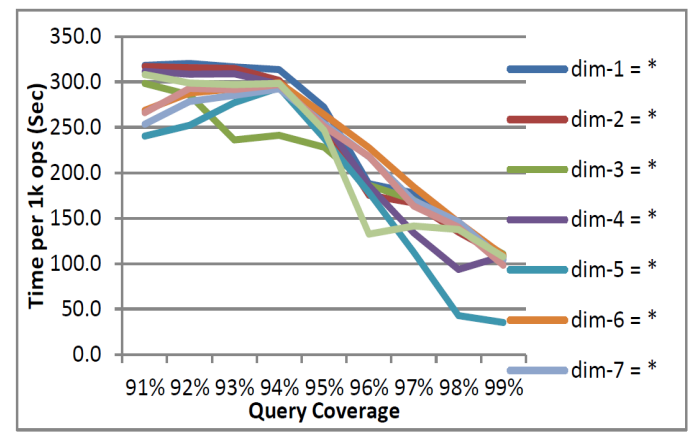

Figure 7.10: Time for 1000 queries as the query coverage in PDCR-tree Impact of queries having value "** for different dimensions. $(N=40 M, m=8, k=$ $16, d=8,91 \%<=$ Coverage $<=99 \%)$

Figure 7.9 and 7.10 show the CR-OLAP is efficient with either very low or very high query coverage. When the query with a low coverage like $10 \%, 20 \%$ or $30 \%$, there are not too many directory nodes whose MDSRs intersect the MDSs of a query, so the PDCR-tree only traverses a small amount of subtrees; When the query has a very large coverage like $95 \%$ to $99 \%$, the results should be containing a large portion of the data in the database. When traversing the PDCR-tree, the MDS of a query covers the MDSRs of many directory nodes in the top part of tree and the aggregation value stored in those directory nodes can 


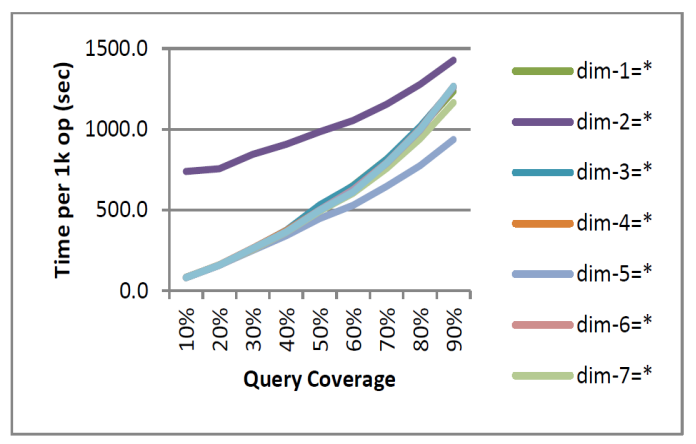

Figure 7.11: Time for 1000 queries as a function of query coverages in 1DIndex Impact of queries having value "*" for different dimensions. $(N=40 M, m=$ $8, k=16, d=8,10 \%<=$ Coverage $<=$ $90 \%)$

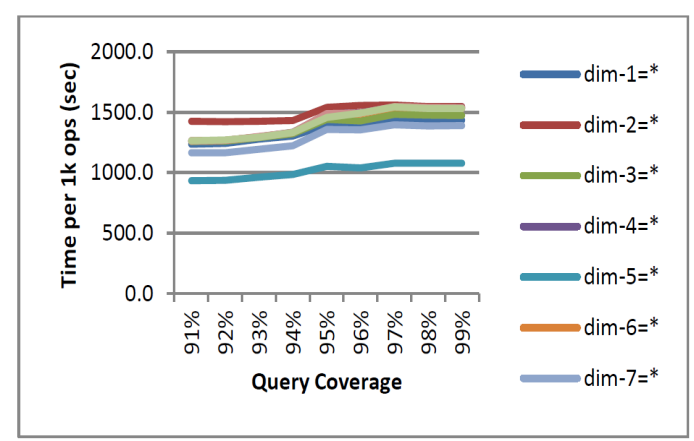

Figure 7.12: Time for 1000 queries as the query coverage in 1D-Index Impact of queries having value "*" for different dimensions. $(N=40 M, m=8, k=$ $16, d=8,91 \%<=$ Coverage $<=99 \%$ )

be reported as a part of results. The PDCR-tree doesn't need tracing down very deep in the tree. However, the 1D-index does not have such advantage. The higher coverage the query result has, the more data nodes in the arrays need to be scanned. As a result, the performance is constantly decreased by increasing the query coverage. (see figure 7.11, and 7.12)

Comparing the PDCR-index to the 1D-tree, the performance of a PDCR-tree is at least 5 to 20 times faster than the performance of a 1D-index is in most cases. The Figure 7.13 and 7.14 show the ration of the query time of $1 \mathrm{D}$-index over the query time of the PDCR-tree. It proves that the performance of the CR-OLAP beats the performance of the Stream-OLAP.

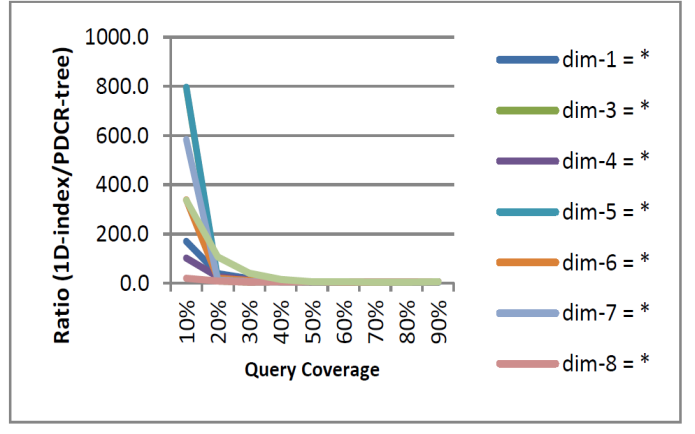

Figure 7.13: Ratio of 1D-index/PDCRtree taken for 1000 queries as the query coverage increases

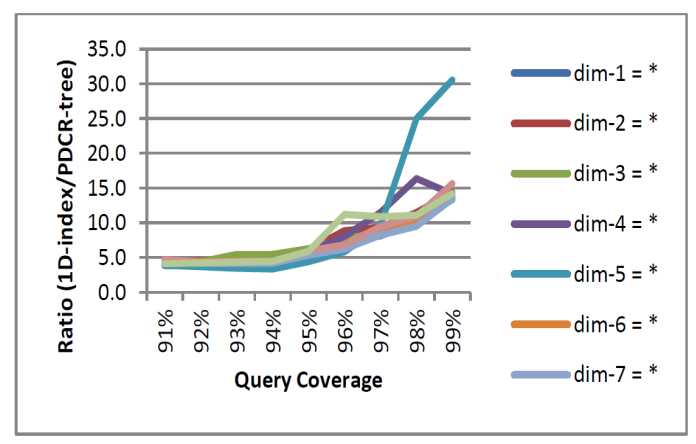

Figure 7.14: Ratio of 1D-index/PDCRtree taken for 1000 queries as the query coverage increases 


\section{Test 5: Varying query pattern with star at different hierarchy levels}

This test is used to evaluate the performance of selected query patterns. It is performed on 40 million tuples from the fact table $(\mathrm{N}=40 \mathrm{M})$ with 8 dimensions $(\mathrm{d}=8)$ as initial insertions. The number of workers is $8(\mathrm{~m}=8)$. The query sets include queries having one or more "*" at different hierarchy levels in the "Date_Dim" dimension. We selected 7 combinations of "*”" and values in the hierarchy levels "Year", "Month" and "Day" as the query patterns for our test. They are $*_{-} *_{-} *$, year-*_*, year-month-*, year-month-day, *- $_{-}$ month-*, *-month-day and *-*-day. Those query patterns cover many OLAP queries such as "Total sales in stores located in Ontario and Alberta from February to May of all years" or "Total sales in all stores in Ottawa in May 2012" etc. We generated 3 sets of queries for each pattern with the coverage $10 \%, 60 \%$ and $95 \%$ respectively.

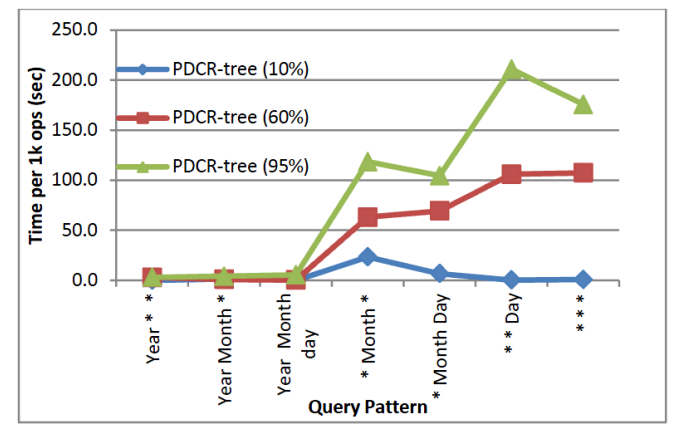

Figure 7.15: Time for 1000 queries as a function of query coverage for queries with multiple “*” values for PDC-tree. $(N=40 M, m=8, k=16, d=$ 8)

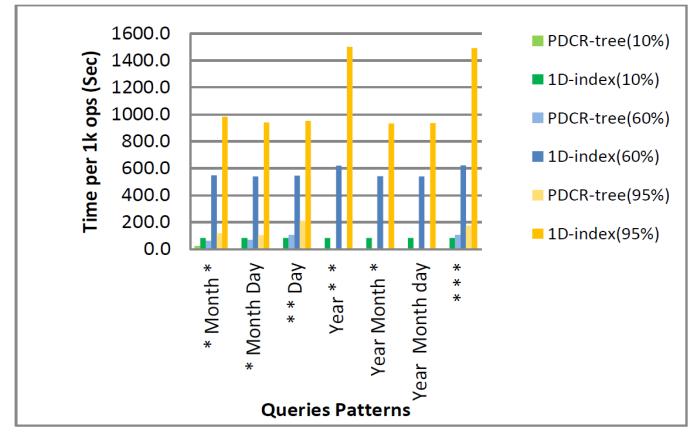

Figure 7.16: Time for 1000 queries as a function of query coverages for queries with multiple "**" values for both PDCRtree and 1D-index. $(N=40 M, m=8, k=$ $16, d=8)$

From figure 7.15, we observe that when the query has value in a higher hierarchy level, the CR-OLAP performs better. The reason is: when the MDS Ids for a query contain values only in higher hierarchies such as "Year-*-*", the MDSs of the query fully cover the MDSRs of directory nodes in the top part of the PDCR-tree. Therefore, the search returns the aggregation value stored in the directory nodes and does not go down to children nodes for further search. However, when the MDS Ids for a query contain values only in lower hierarchies such as "*-*-Day", the MDSs can have many intersection with the MDSRs of directory nodes. Therefore, it needs to traverse the tree to the very bottom part in order 
to filter the data for a specific day of every year and every month. This becomes more obviously when the coverage is very high (e.g. 95\%). Figure 7.16 also demonstrates that the PDCR-tree consistently outperforms the 1D-index no matter what query coverage and query patterns are. Therefore, the CR-OLAP system has the advantage to support all kinds of OLAP queries and operations.

\section{Test 6: Changing the type of cloud instance}

This test is used to evaluate the CR-OLAP performance on different types of cloud instances. Five types of instances are selected and they are the VCL HPC instance, the Amazon cc2.8xlarge, cr1.8xlarge, m3.2xlarge and m2.4xlarge. The experiments are performed with initial 10 million data $(\mathrm{N}=10 \mathrm{M})$ with 8 dimensions $(\mathrm{d}=8)$ as insertions followed by three sets of 1000 queries ( $q=1000)$ with $10 \%, 60 \%$ and $95 \%$ coverage respectively. We built the system on 5 different instances and each time created 5 nodes including one master and 4 workers.

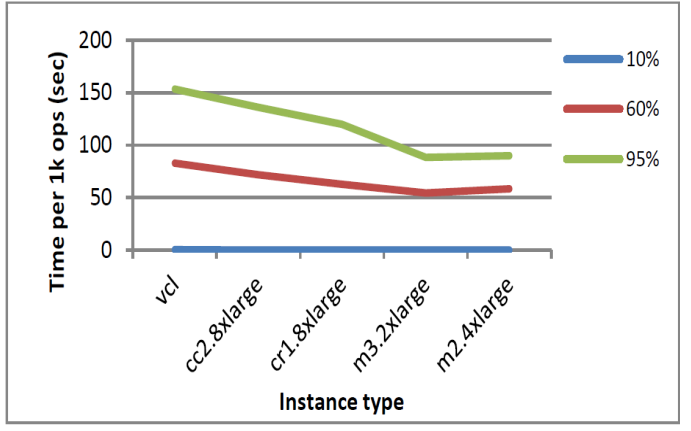

Figure 7.17: Time for 1000 queries on different type cloud instances. $(N=$ $10 M, m=4, k=16)$

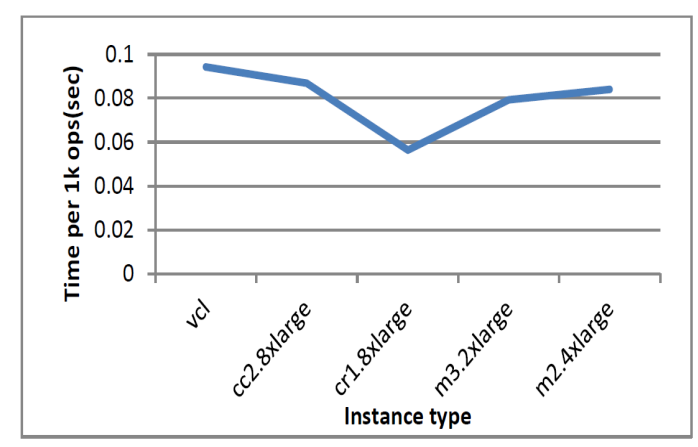

Figure 7.18: Time for 1000 insertions on different type cloud instances. $(N=$ $10 M, m=4, k=16)$

Figure 7.17 and 7.18 show that the CR-OLAP is running faster on the Amazon cloud than it is on the VCL cloud. Amazon defines an EC2 computing unit (ECU) to measure its CPU capacity. Each ECU provides the equivalent CPU capacity of a 1.0-1.2 GHz 2007 Opteron or 2007 Xeon processor [2]. For example, an m2.4xlarge instance has 8 virtual CPUs with 3.25 ECUs on each CPU, so each CPU speed is 3.98GHz. The VCL HPC instance has 16 cores and each cpu speed is $2.1 \mathrm{GHz}$. The better CPU leads to better performance of the CR-OALP on the Amazon cloud. The Amazon cloud also provides better 
networking performance than the VCL does so it benefits the CR-OLAP for transferring data or messages between instances.

In addition, the same figure also illustrates that the CR-OLAP performs better on $\mathrm{m} 2.4 x$ large instances and an m3.2xlarge instances than it does on cc2.8xlarge instances and cr1.8xlarge instances. The cluster type instances such as cc2.8xlarge and cr1.8xlarge have 88 ECUs for 32 virtual CPUs. Each CPU has the computing capability of 2.75 ECUs. The CPU speed of the cluster type instances is about $3.3 \mathrm{GHz}$ which is slower than the CPU capability of an m2.4xlarge or an m3.2xlarge instance. Cluster instances can be built within one placement group which provides non-blocking 10 Gigabit Ethernet network. Since our tests are using 16 threads and most data and messages including migration subtrees transferring between the instances are not very large, so those experiments didn't actually take the advantage of high cores and high network speed of the cluster instances. We expect when the number of threads is increased in the system or the data size is increased, the cluster instances can outperform the regular instances.

\section{Test 7: Increasing number of cut level}

The test is used to evaluate the impact of the cut level. The experiments are performed with an initial 10 million data $(\mathrm{N}=10 \mathrm{M})$ with 8 dimensions $(\mathrm{d}=8)$ as insertions followed by 1000 queries ( $q=1000)$ with $10 \%$ coverage. The cut level is the depth of the hat in a PDCR-tree on a master node. We increase the cut level from 1 to 10.

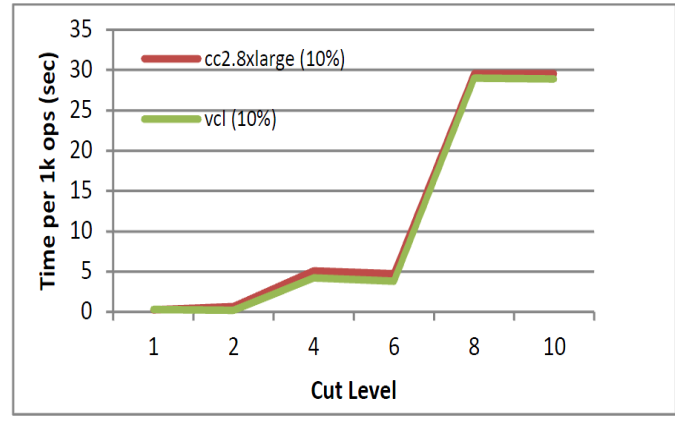

Figure 7.19: Time for 1000 queries as a function of the number of cut level in hat. $(N=10 M, m=4, k=16)$

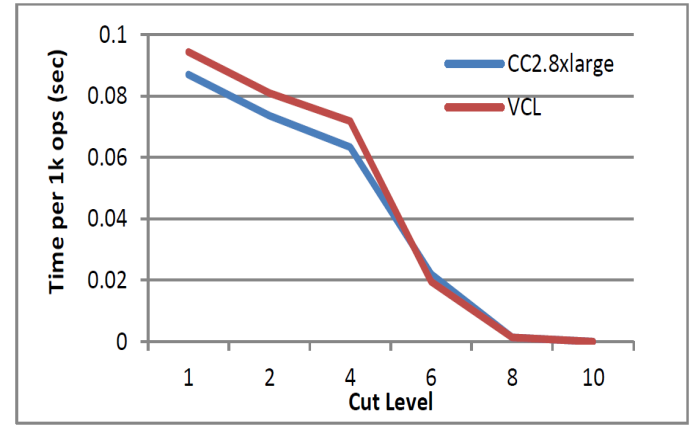

Figure 7.20: Time for 1000 insertions as a function of the number of cut level in hat. $(N=10 M, m=4, k=16)$

The Figure 7.19 and 7.20 show that the better performance can be achieved when the 
cut level is smaller. The system is designed to use multiple parallelism including parallel computing by multiple workers and parallel multi-threads on each worker. The CR-OLAP distributes the data to multiple workers, therefore, insertions and queries can be dispatched to multiple workers to be executed concurrently not only within a worker but also on multiple workers in order to improve computing speed. When we increase the cut level in the hat, the tree on the master becomes larger and the processing time of traversing the hat is increased unless we use more threads in the processing. To use more threads, we need to improve the hardware of the master node (e.g.increasing the number of cores) which is not easy to achieve sometimes.

\section{Test 8: Increasing directory node capacity}

This test is used to evaluate the impact of the directory node capacity. The experiments are performed with an initial 10 million data $(\mathrm{N}=10 \mathrm{M})$ with 8 dimensions $(\mathrm{d}=8)$ as insertions followed by four sets of 1000 queries ( $q=1000)$ with 10\%, 20\%, 60\% and 95\% coverage respectively. We performed two experiments by varying the node capacity from 10 to 35 in the hat only and in the workers only as well. When changing the capacity in the hat, the directory node capacity in the workers is fixed to 15 , while changing the capacity in the workers, the node capacity in the hat is fixed to 10.

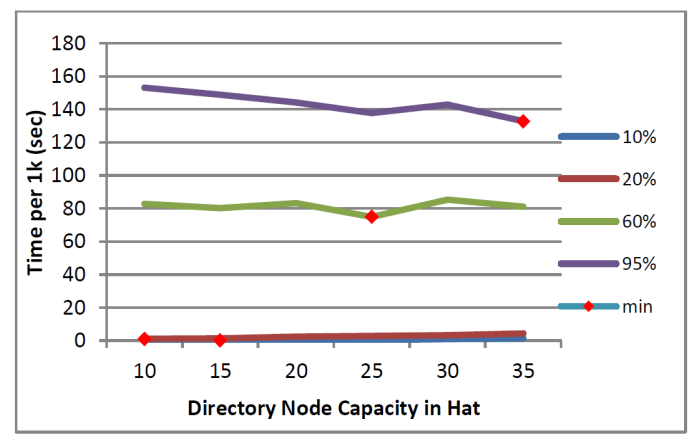

Figure 7.21: Time for 1000 queries as a function of the number of directory node capacity in hat. $(N=10 M, m=4, k=16)$

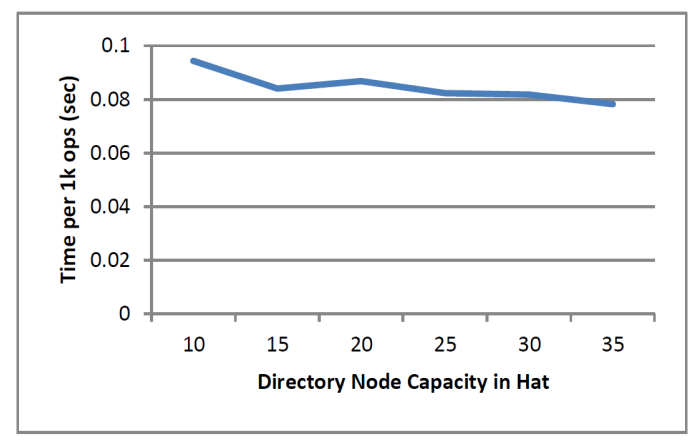

Figure 7.22: Time for 1000 insertions as a function of the number of directory node capacity in hat. $(N=10 M, m=$ $4, k=16)$

We notice that when increasing the node capacity of directory nodes in the hat, the query time is decreased in a high data coverage but increased in a low coverage. When the directory node capacity is increased, the tree increases its flatness and contains more 
directory/data nodes in the hat. Figure 7.21 demonstrates that for a low coverage, the query traverses more nodes in the hat so it slows down the search process; for a high coverage, the query can get results from certain directory nodes in the hat if the MDSRs is fully covered within query's MDSs so it speeds up the performance. Figure 7.22 shows the insertion time is improved when the node capacity is increased since less node splits happen during insertions.

We have illustrated the impact of the node capacity in a PDC-tree on multi-core processors. The capacity of a directory node in the PDC-tree can be optimized to have the best performance. The PDCR-tree also carries such character. When we ran the test with node capacity 40 and the query time went up to over 1000 seconds for queries with any coverage. Therefore, for the fixed parameter settings provided in this test, the system has the optimal query performance for queries in the case of a high coverage when the directory node capacity is around 25 to 35; the optimal query performance for queries in the case of a low coverage when the node capacity is around 10 to 15 . The optimal insertion output is reached when the directory node capacity is near 35 .

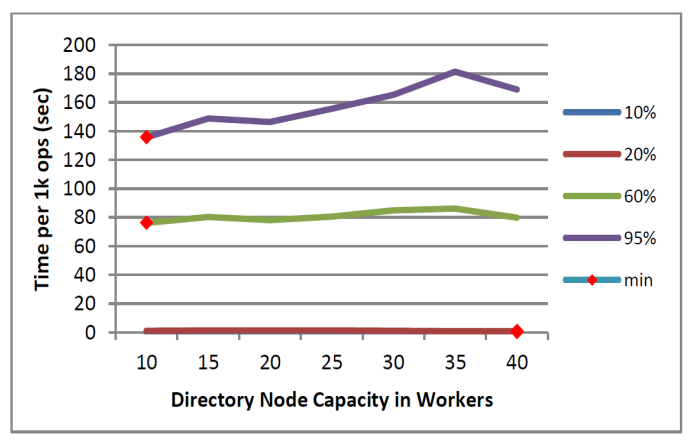

Figure 7.23: Time for 1000 queries as a function of the number of directory node capacity in workers. $(N=10 M, m=$ $4, k=16)$

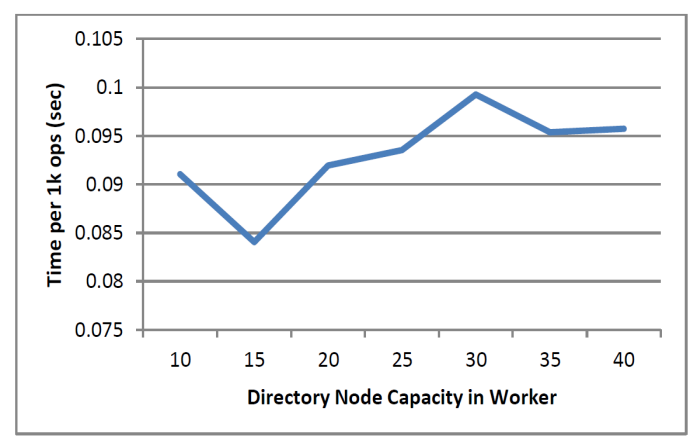

Figure 7.24: Time for 1000 insertions as a function of the number of directory node capacity in workers. $(N=10 M, m=$ $4, k=16)$

From figure 7.23, for a fixed directory node capacity 10 in the hat, we observe that the PDCR-tree reaches its optimal output when the directory node capacity in the workers is near 10 for queries with a high coverage and is around 40 for queries with a low coverage. For insertions, the experiment shows the result reach the best at the capacity of 15 (Figure 7.24). 
Furthermore, we also tested the system by increasing the directory node capacity at the same time in both hat and workers. The experiment (Figure 7.25 and 7.26) shows that the capacity of 10-15 leads to the best performance of the CR-OLAP system for query operations and the capacity of 15 results in the best performance for insertions.

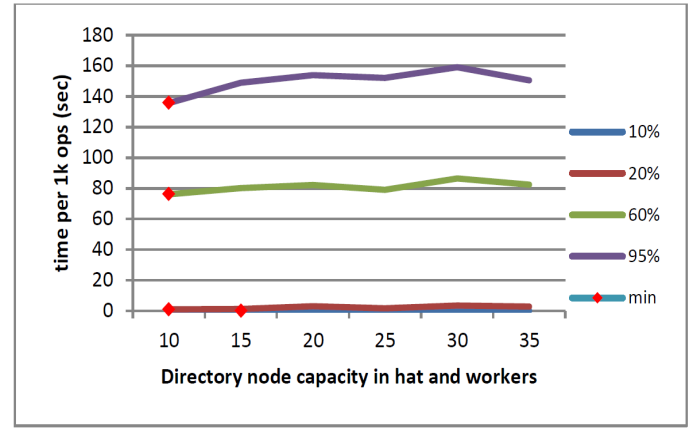

Figure 7.25: Time for 1000 queries as a function of the number of directory node capacity in both hat and workers. $(N=$ $10 M, m=4, k=16)$

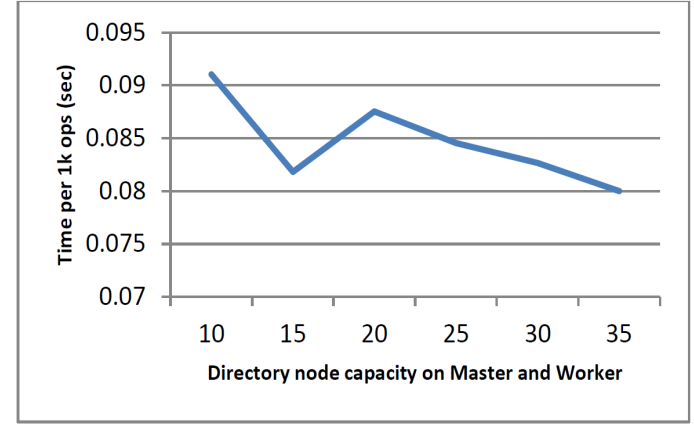

Figure 7.26: Time for 1000 insertions as a function of the number of directory node capacity in both hat and workers. $(N=10 M, m=4, k=16)$

With the above tests, we know that when changing the value of parameters like a cut level and a directory node capacity, the CR-OLAP can have different behaviors. With a fixed 10 million data set, the system can reach the best output with the directory node capacity around 10-15 at the cut level 1 or 2 . When data size is changed, the values of each parameter can be various to obtain the best results. Therefore, these parameters can be tuned to optimize the system performance. 


\section{Chapter 8}

\section{Conclusions}

\subsection{Conclusions}

Business Intelligence and its tools such as data warehouses, OLAP servers and report systems have their important roles in organizations to assist in making decision precisely and reacting to market changing quickly. With the increasing globalization of market and the growing complexity of businesses, there is an increasing demand on BI and OLAP systems. The current OLAP systems have been facing challenges such as storage spaces for an increasing data size, query responses for an efficient performance, or data freshness for analysis in real-time. In many common practices, an OLAP server loads data periodically to its data cubes. The loading procedures usually can take hours to be completed since many extra data and calculations are added during loading. The cube contains mostly static and pre-aggregated data but provides multi-dimensional views to users. A such fashion cannot meet the needs of modern businesses. Many decision makers are seeking real-time OLAP systems which can provide the analysis with integrated data source in real-time such that the results are not based on outdated information. However, it is a challenge to provide such a system which involves large portion data aggregation and responses user requests with low latency and high throughput.

\subsection{Summary of Contributions}

In this thesis, we proposed a real-time OLAP system on a cloud environment (CR-OLAP) as a solution. The system uses a new distributed index, a distributed PDCR-tree, for multidimensional databases to enhance the system performance. The PDCR-tree is based on the previous studies of the sequential DC-tree and the PDC-tree on multi-core processor. It supports multi-dimensional databases and allows incoming insertions and queries to be performed in parallel in a distributed environment. The system is built on the cloud using 
$\mathrm{m}+1$ machine nodes and each machine may have multiple cores to run up to $\mathrm{k}$ threads. Using the PDCR-tree index, data can be partitioned and distributed among $\mathrm{m}$ workers. Except storing data, each worker also stores several subtrees of the PDCR-tree. The ' 1 ' in ' $\mathrm{m}+1$ ' is referred to a master that contains the hat of the PDCR-tree. The master receives a stream of insertion and query requests from users and dispatches the tasks to various workers. Results returned by each worker will be aggregated in the master and reported to users by the master. In order to achieve an optimal performance, queries and insertions are not only processed among multiple workers in parallel, but also executed in parallel within each worker. The data structure of the PDCR-tree supports both parallelism.

The CR-OLAP system supports various range aggregation queries. A value can be specified in each hierarchy level in a query. Also a star "*” can be indicated in any level of hierarchies in the query to represent ALL values. We designed MDS Ids using bits to allow such representation. The ids are also used to represent conceptual hierarchies of each dimension in different levels for data nodes in the PDCR-tree. The bit representation in ids allow us to define the range of MDSs for unordered and ordered dimensions so that it improves the system performance.

We evaluated the performance of the PDCR-tree using the TPC-DS decision support benchmark data set on the Amazon EC2 cloud and the VCL cloud for multiple scenarios. The experiments show that the system is able to be scaled up for the growth of data. While the number of workers is increased, the query throughput is improved. For example, for 10 million data tuples, while increasing the number of workers from 1 to 8 , the average query throughput is increased by a factor 7.5. The system is especially efficient to answer queries with a high data coverage. For example, to perform a range query with $60 \%$ coverage on a database with 80 million tuples using 8 workers, the result can be returned in 0.3 seconds. This is considered as a response in real time .

\subsection{Future Research}

The system can still be improved to enhance its performance. The load balancer can be optimized. In currently implementation, the load balancer only checks the memory usage with a pre-defined threshold. We have discussed to use a dynamic load balancer that periodically calculates the variance of memory usages and subtrees work loads on each worker. 
The system can use the variance as a dynamic threshold for a certain time period to trigger migration. This has not been implemented. In addition, we maintain all information of subtrees and workers on the master node in the existing CR-OLAP. We originally designed it to use the Apache Zookeeper in an additional machine to maintain the information of distributed subtrees and workers so it can avoid losing the information if the master node fails. The Zookeeper is a centralized service that synchronizes information used in distributed systems. When the CR-OLAP is scaled up or if the system is modified to use multiple master nodes, it needs a good coordination service to maintain system information like the mapping of workers and subtrees, workers working on the same queries or the file directories storing results etc. We tested the system with the zookeeper on the VCL Cloud, the speed (80ms per read operation) was not satisfied so it was not fully implemented. However, we tested it on the Amazon cloud and found it was very fast (1ms) on read operations. Therefore, The Zookeeper can be added to systems to avoid losing information from node failures. Last but not least, a data replication can be included into the system. This also has to do with node failures. The data replication can be used to restore the data if any node failure happens so that user requests do not get disturbed. This is extremely important to a real-time OLAP system that supports businesses such as stock exchange analysis. 


\section{Bibliography}

[1] 0mq - the guide. http://zguide.zeromq.org/page:all.

[2] Amazon ec2 instance details. http://aws.amazon.com/ec2/.

[3] Amazon elastic compute cloud (amazon ec2). http://aws.amazon.com/ec2/.

[4] Big data meets big data analytics. White Paper.

[5] Improve decision making with dss - dcision support systems. http://www.targit.com/en/LandingPages/DecisionSupportSystem/DSS.

[6] Introduction to business intelligence architecture. http://www.scribd.com/doc/18511802/Module-1-Introduction-to-BusinessIntelligence-Architecture.

[7] Olap council white paper. http://www.olapcouncil.org/research/whtpaply.htm.

[8] Olap cube. http://apandre.wordpress.com/data/datacloud/datacube/.

[9] Oltp vs. olap. http://datawarehouse4u.info/OLTP-vs-OLAP.html.

[10] Oracle8 parallel server concepts \& administration. http://docs.oracle.com/cd/A58617_01/server.804/a58238/ch1_unde.htm.

[11] Outperform your competition with powerful data insights.

[12] R-tree. http://en.wikipedia.org/wiki/R-tree.

[13] Why is business intelligence so important. http://bdaily.co.uk/advice/09-022012/why-is-business-intelligence-so-important/.

[14] Bi: Dimensional model - star schema. http://blogmstechnology.blogspot.ca/2010/06/bi-dimensional-model-star-schema.html, 2010 .

[15] U. Ahmed, A. Tchounikine, M. Miquel, and S. Servigne. Real-time temporal data warehouse cubing. pages 159-167, 2010.

[16] H. Al-Aqrabi, L. Liu, R. Hill, and N. Antonopoulos. Taking the business intelligence to the clouds. In High Performance Computing and Communication \& 2012 IEEE 9th International Conference on Embedded Software and Systems (HPCC-ICESS), 2012 IEEE 14th International Conference on, pages 953-958. IEEE, 2012. 
[17] K. Asanovic, R. Bodik, B. C. Catanzaro, J. J. Gebis, P. Husbands, K. Keutzer, D. A. Patterson, W. L. Plishker, J. Shalf, S. W. Williams, et al. The landscape of parallel computing research: A view from berkeley. Technical report, Technical Report UCB/EECS-2006-183, EECS Department, University of California, Berkeley, 2006.

[18] S. Berchtold, D. A. Keim, and H.-P. Kriegel. The x-tree: An index structure for highdimensional data. Readings in multimedia computing and networking, page 451, 2001.

[19] P. Brezany, Y. Zhang, I. Janciak, P. Chen, and S. Ye. An elastic olap cloud platform. In Dependable, Autonomic and Secure Computing (DASC), 2011 IEEE Ninth International Conference on, pages 356-363. IEEE, 2011.

[20] Y. Cao, C. Chen, F. Guo, D. Jiang, Y. Lin, B. C. Ooi, H. T. Vo, S. Wu, and Q. Xu. Esi $\sup _{i} 2 i / \sup _{i}$ : A cloud data storage system for supporting both oltp and olap. In Data Engineering (ICDE), 2011 IEEE 27th International Conference on, pages 291-302. IEEE, 2011.

[21] S. Chaudhuri and U. Dayal. An overview of data warehousing and olap technology. ACM Sigmod record, 26(1):65-74, 1997.

[22] C. Chen, G. Chen, D. Jiang, B. C. Ooi, H. T. Vo, S. Wu, and Q. Xu. Providing scalable database services on the cloud. In Web Information Systems Engineering-WISE 2010, pages 1-19. Springer, 2010.

[23] Y. Chen, F. Dehne, T. Eavis, and A. Rau-Chaplin. Parallel rolap data cube construction on shared-nothing multiprocessors. In Parallel and Distributed Processing Symposium, 2003. Proceedings. International, pages 10-pp. IEEE, 2003.

[24] S. S. Conn. Oltp and olap data integration: a review of feasible implementation methods and architectures for real time data analysis. In SoutheastCon, 2005. Proceedings. IEEE, pages 515-520. IEEE, 2005.

[25] J. Dai. Efficient concurrent operations in spatial databases. PhD thesis, Virginia Polytechnic Institute and State University, 2009.

[26] F. Dehne. Parallel data cube. lecture notes.

[27] F. Dehne, T. Eavis, S. Hambrusch, and A. Rau-Chaplin. Parallelizing the data cube. Distributed and Parallel Databases, 11(2):181-201, 2002.

[28] F. Dehne, T. Eavis, and A. Rau-Chaplin. A cluster architecture for parallel data warehousing. In Cluster Computing and the Grid, 2001. Proceedings. First IEEE/ACM International Symposium on, pages 161-168. IEEE, 2001.

[29] F. Dehne, T. Eavis, and A. Rau-Chaplin. Coarse grained parallel on-line analytical processing (olap) for data mining. In Computational Science-ICCS 2001, pages 589598. Springer, 2001. 
[30] F. Dehne, T. Eavis, and A. Rau-Chaplin. Computing partial data cubes for parallel data warehousing applications. In Recent Advances in Parallel Virtual Machine and Message Passing Interface, pages 319-326. Springer, 2001.

[31] F. Dehne, T. Eavis, and A. Rau-Chaplin. Distributed multi-dimensional rolap indexing for the data cube. In The 3rd IEEE/ACM International Symposium on Cluster Computing and the Grid (CCGrid 2003), 2003.

[32] F. Dehne, T. Eavis, and A. Rau-Chaplin. Top-down computation of partial rolap data cubes. In System Sciences, 2004. Proceedings of the 37th Annual Hawaii International Conference on, pages 10-pp. IEEE, 2004.

[33] F. Dehne and H. Zaboli. Parallel real-time olap on multi-core processors. In Proceedings of the 2012 12th IEEE/ACM International Symposium on Cluster, Cloud and Grid Computing (ccgrid 2012), pages 588-594. IEEE Computer Society, 2012.

[34] L. dOrazio and S. Bimonte. Multidimensional arrays for warehousing data on clouds. In Data Management in Grid and Peer-to-Peer Systems, pages 26-37. Springer, 2010.

[35] C. Dubler and C. Wilcox. Just what are cubes anyway?(a painless introduction to olap technology). Microsoft Corporation, Abril de, 2002.

[36] M. Ester, J. Kohlhammer, and H.-P. Kriegel. The dc-tree: A fully dynamic index structure for data warehouses. In Data Engineering, 2000. Proceedings. 16th International Conference on, pages 379-388. IEEE, 2000.

[37] H. Gao and J.-Z. Li. Parallel data cube storage structure for range sum queries and dynamic updates. Journal of Computer Science and Technology, 20(3):345-356, 2005.

[38] S. Goil and A. Choudhary. Parallel data cube construction for high performance on-line analytical processing. In High-Performance Computing, 1997. Proceedings. Fourth International Conference on, pages 10-15. IEEE, 1997.

[39] S. Goil and A. Choudhary. A parallel scalable infrastructure for olap and data mining. In Database Engineering and Applications, 1999. IDEAS'99. International Symposium Proceedings, pages 178-186. IEEE, 1999.

[40] A. Guttman. R-trees: a dynamic index structure for spatial searching, volume 14. ACM, 1984.

[41] D. Hoksza and T. Skopal. Native multidimensional indexing in relational databases.

[42] K. Jaladi. Data warehousing: Data models and olap operations. www.cacs.louisiana.edu/ cmps566/Notes. 
[43] D. Jin and T. Tsuji. Parallel data cube construction based on an extendible multidimensional array. In Trust, Security and Privacy in Computing and Communications (TrustCom), 2011 IEEE 10th International Conference on, pages 1139-1145. IEEE, 2011.

[44] K. Jones. An introduction to data warehousing: What are the implications to the network? International Journal of Network Management, 8(1):42-56, 1998.

[45] R. Kalakota. Gartner says bi and analytics a $\$ 12.2$ bln market. practicalanalytics.wordpress.com/2011/04/24/gartner-says-bi-and-analytics-a10-5-bln-market/, 2011.

[46] R. Kalakota. Ibm cio study: $\mathrm{Bi}$ and analytics are \#1 priority for 2012/2013. practicalanalytics.wordpress.com/2011/11/02/ibm-cio-study-bi-andanalytics-are-1-priority-for-2012/, 2011.

[47] H. Luan, X.-Y. Du, and S. Wang. Cache-conscious data cube computation on a modern processor. Journal of Computer Science and Technology, 24(4):708-722, 2009.

[48] H. P. Luhn. A business intelligence system. IBM Journal of Research and Development, 2(4):314-319, 1958.

[49] H. Mailvaganam. Introduction to olap - slice, dice and drill! Data Warehousing Review, 2007.

[50] V. Markl, F. Ramsak, and R. Bayer. Improving olap performance by multidimensional hierarchical clustering. In Database Engineering and Applications, 1999. IDEAS'99. International Symposium Proceedings, pages 165-177. IEEE, 1999.

[51] R. Mulcahy. Business intelligence definition and solutions.

[52] L. S. Nyland, J. F. Prins, A. Goldberg, and P. H. Mills. A design methodology for data-parallel applications. Software Engineering, IEEE Transactions on, 26(4):293$314,2000$.

[53] C. Olston, B. Reed, U. Srivastava, R. Kumar, and A. Tomkins. Pig latin: a not-soforeign language for data processing. In Proceedings of the 2008 ACM SIGMOD international conference on Management of data, pages 1099-1110. ACM, 2008.

[54] D. Power. A brief history of decision support systems, version 4.0, march 10, 2007. Series A Brief History of Decision Support Systems. Version, 4, 2007.

[55] D. J. Power. A brief history of decision support systems, version 4.0. DSSResources.COM, 2007. 
[56] K. Ravi Kanth, D. Serena, and A. K. Singh. Improved concurrency control techniques for multi-dimensional index structures. In Parallel Processing Symposium, 1998. IPPS/SPDP 1998. Proceedings of the First Merged International... and Symposium on Parallel and Distributed Processing 1998, pages 580-586. IEEE, 1998.

[57] R. J. Santos and J. Bernardino. Real-time data warehouse loading methodology. pages 49-58, 2008.

[58] R. J. Santos and J. Bernardino. Optimizing data warehouse loading procedures for enabling useful-time data warehousing. In Proceedings of the 2009 International Database Engineering \& Applications Symposium, pages 292-299. ACM, 2009.

[59] S. I. Song, Y. H. Kim, and J. S. Yoo. An enhanced concurrency control scheme for multidimensional index structures. Knowledge and Data Engineering, IEEE Transactions on, 16(1):97-111, 2004.

[60] A. Thusoo, J. S. Sarma, N. Jain, Z. Shao, P. Chakka, N. Zhang, S. Antony, H. Liu, and R. Murthy. Hive-a petabyte scale data warehouse using hadoop. In Data Engineering (ICDE), 2010 IEEE 26th International Conference on, pages 996-1005. IEEE, 2010.

[61] R. K. Upadhyay. Olap in business intelligence.

[62] R. K. Upadhyay. Idc: Analytics a \$51b business by 2016 thanks to big data. gigaom.com/2012/07/11/idc-analytics-a-51b-business-by-2016-thanks-to-bigdata/, 2012.

[63] F. Willmore. Introduction to parallel computing. http://www.tacc.utexas.edu.

[64] Y. Zhai, M. Liu, J. Zhai, X. Ma, and W. Chen. Cloud versus in-house cluster: evaluating amazon cluster compute instances for running mpi applications. In State of the Practice Reports, page 11. ACM, 2011.

[65] X. Zhang, J. Ai, Z. Wang, J. Lu, and X. Meng. An efficient multi-dimensional index for cloud data management. In Proceedings of the first international workshop on Cloud data management, pages 17-24. ACM, 2009.

[66] Y. Zhang, S. Wang, and W. Huang. Paracube: A scalable olap model based on distributed aggregate computing with sibling cubes. In Web Conference (APWEB), 2010 12th International Asia-Pacific, pages 323-329. IEEE, 2010. 